\title{
Supersymmetry breaking branes on solvmanifolds and de Sitter vacua in string theory
}

\author{
David Andriot, ${ }^{a}$ Enrico Goi, ${ }^{b}$ Ruben Minasian $^{b}$ and Michela Petrini ${ }^{a}$ \\ ${ }^{a}$ LPTHE, CNRS, UPMC Université de Paris 06, \\ Bô̂te 126, 4 Place Jussieu, F-75252 Paris cedex 05, France \\ ${ }^{b}$ Institut de Physique Théorique, CEA/Saclay, \\ 91191 Gif-sur-Yvette Cedex, France \\ E-mail: andriot@lpthe.jussieu.fr, enrico.goi@cea.fr, \\ ruben.minasian@cea.fr, petrini@lpthe.jussieu.fr
}

ABSTRACT: We consider IIA compactifications on solvmanifolds with O6/D6 branes and study the conditions for obtaining de Sitter vacua in ten dimensions. While this is a popular set-up for searching de Sitter vacua, we propose a new method to include supersymmetry breaking sources. For space-time filling branes preserving bulk supersymmetry, the energy density can easily be extremized with respect to all fields, thanks to the replacement of the DBI action by a pullback of a special form given by a pure spinor. For sources breaking bulk supersymmetry, we propose to replace the DBI action by the pullback of a more general polyform, which is no longer pure. This generalization provides corrections to the energy-momentum tensor which give a positive contribution to the cosmological constant. We find a de Sitter solution to all (bulk and world-volume) equations derived from this action. We argue it solves the equations derived from the standard source action. The paper also contains a review of solvmanifolds.

KEYwords: Flux compactifications, dS vacua in string theory, Differential and Algebraic Geometry, Superstring Vacua

ARXIV EPRINT: 1003.3774 


\section{Contents}

1 Introduction 1

2 Supersymmetric backgrounds, solvmanifolds and twist transformations 8

$\begin{array}{ll}2.1 \text { Twist construction of (almost abelian) solvmanifolds } & 11\end{array}$

$\begin{array}{ll}2.2 & \text { Twist transformations in generalized geometry } \\ \end{array}$

2.3 Type IIA supersymmetric solutions from twist transformations 18

$\begin{array}{lll}2.3 .1 & \text { Localizing the sources and warping } & 21\end{array}$

2.4 A digression: twist and non-geometric backgrounds 22

3 Supersymmetry breaking and de Sitter vacua $\quad 24$

$\begin{array}{lll}3.1 & \text { Solvable de Sitter } & 29\end{array}$

$\begin{array}{lll}3.1 .1 & \text { The solution } & 30\end{array}$

3.2 More on the polyform $X \quad 34$

$\begin{array}{lll}3.3 & \text { A solution for the standard source action? } & 38\end{array}$

$\begin{array}{lll}3.4 & \text { Four-dimensional analysis } & 40\end{array}$

3.4.1 Moduli and 4d Einstein frame 40

$\begin{array}{lll}3.4 .2 & \text { Extremization and stability } & 42\end{array}$

A Solvable algebras and the geometry of solvmanifolds $\quad 45$

$\begin{array}{lll}\text { A.1 Algebraic aspects } & 45\end{array}$

$\begin{array}{lll}\text { A.1.1 The adjoint action } & 46\end{array}$

$\begin{array}{lll}\text { A.1.2 Semidirect products } & 47\end{array}$

$\begin{array}{lll}\text { A.1.3 Solvable groups } & 48\end{array}$

$\begin{array}{lll}\text { A.2 Compactness } & 49\end{array}$

A.2.1 Algebras admitting a lattice $\quad 52$

A.3 Algorithmic construction of the one-forms of a solvable group 52

A.3.1 Mostow bundle structure: $A_{M} \quad 53$

$\begin{array}{ll}\text { A.3.2 Nilmanifold fibration structure: } A_{N} & 54\end{array}$

A.4 Six-dimensional solvmanifolds in terms of globally defined one-forms $\quad 56$

$\begin{array}{ll}\text { B T-dualising solvmanifolds } & 57\end{array}$

\section{Introduction}

In recent years much progress has been achieved in the classification and construction of supersymmetric flux compactifications [1-3]. This is largely due to the fact that, at least for type II supergravities, supersymmetry allows to look at first order differential equations, which together with the Bianchi identities for the fluxes, imply the solutions of 
the ten-dimensional equations of motion [4-6]. A natural frame to analyse backgrounds with $\mathcal{N}=1$ supersymmetry is provided by generalized complex geometry (GCG) [7, 8], which was developed concurrently with the progress on the physics side of the problem. In this formalism, the natural variables are a certain combination of globally defined even or odd differential forms, $\Phi_{ \pm}$, called pure spinors, and the supersymmetry conditions amount to a set of differential equations for such spinors. For $\mathcal{N}=1$ compactifications to fourdimensional Minkowski space these are

$$
\begin{aligned}
\mathrm{d}_{H}\left(e^{2 A-\phi} \Phi_{1}\right) & =0, \\
\mathrm{~d}_{H}\left(e^{A-\phi} \operatorname{Re} \Phi_{2}\right) & =0, \\
\mathrm{~d}_{H}\left(e^{3 A-\phi} \operatorname{Im} \Phi_{2}\right) & =\frac{|a|^{2}}{8} e^{3 A} * \lambda(F) .
\end{aligned}
$$

$\Phi_{1}=\Phi_{ \pm}$and $\Phi_{2}=\Phi_{\mp}$ for IIA/IIB , where + and - denote even and odd forms, respectively. $\phi$ is the dilaton and $|a|^{2}=\left\|\Phi_{ \pm}\right\|$is the norm of the pure spinors, ${ }^{1}$ which is fixed to $|a|^{2}=e^{A}$. F denotes the sum of the RR fluxes on the internal manifold

$$
\begin{aligned}
& \text { IIA }: F=F_{0}+F_{2}+F_{4}+F_{6}, \\
& \text { IIB }: \quad F=F_{1}+F_{3}+F_{5},
\end{aligned}
$$

and is related to the total ten-dimensional RR field-strength $F^{(10)}$ by

$$
F^{(10)}=F+\operatorname{vol}_{(4)} \wedge \lambda(* F),
$$

where $\operatorname{vol}_{(4)}$ is the warped four-dimensional volume form with warp factor $e^{2 A}$. The NS flux $H$ enters the equations through the differential $\mathrm{d}_{H}=\mathrm{d}-H \wedge$.

The first equation in (1.1) requires the existence on the manifold of a closed pure spinor, and the integrability of the associated generalized complex structure [9, 10]. Spaces admitting a closed pure spinor are generalized Calabi-Yau (GCY). This is, therefore, a necessary condition for preserving supersymmetry. In addition, we should require the existence of a second compatible pure spinor ${ }^{2}$ whose real part is closed, and whose imaginary part is the $\mathrm{RR}$ field. The metric in the internal space is determined by the two pure spinors.

One can see easily that the RR equations of motion automatically follow from the supersymmetry conditions, provided that no NS source is present $(\mathrm{d} H=0)$. Differentiating

\footnotetext{
${ }^{1}$ To define the norm of the pure spinors we introduce the Mukai pairing of two polyforms as the top form:

$$
\left\langle X_{1}, X_{2}\right\rangle=\left.\left(X_{1} \wedge \lambda\left(X_{2}\right)\right)\right|_{\text {top }}
$$

where $\lambda$ acts on any $p$-form $A_{p}$ as the complete reversal of its indices: $\lambda\left(A_{p}\right)=(-1)^{\frac{p(p-1)}{2}} A_{p}$. Then we can define the norm of $\Phi_{ \pm}$as $8\left\langle\Phi_{ \pm}, \bar{\Phi}_{ \pm}\right\rangle=-i\left\|\Phi_{ \pm}\right\|^{2}$ vol. We take the following convention for the Hodge star:

$$
*\left(\mathrm{~d} x^{\mu_{1}} \wedge \ldots \wedge \mathrm{d} x^{\mu_{p}}\right)=\frac{\sqrt{|g|}}{(d-p) !}(-1)^{(d-p) p} \epsilon^{\mu_{1} . . \mu_{p} \mu_{p+1} . . \mu_{d}} g_{\mu_{p+1} \nu_{p+1}} . . g_{\mu_{d} \nu_{d}} \mathrm{~d} x^{\nu_{p+1}} \wedge \ldots \wedge \mathrm{d} x^{\nu_{d}},
$$

with $d$ the dimension of the space, $|g|$ the determinant of the metric. For $\epsilon$ we choose the convention $\epsilon_{1 \ldots d}=1$.

${ }^{2}$ The existence of a pure spinor reduces the structure group on $T M \oplus T^{*} M$ to $\mathrm{SU}(3,3)$. If the manifold admits a second compatible pure spinor the structure group is further reduced to $\mathrm{SU}(3) \times \mathrm{SU}(3)$.
} 
the last equation in (1.1), one indeed recovers the RR flux equations of motion

$$
(\mathrm{d}+H \wedge)\left(e^{4 A} * F\right)=0 .
$$

In a sense, up to this point, finding a supersymmetric string background is a perfectly algorithmic procedure. Indeed, starting from a generalized CY structure, i.e. a twisted closed pure spinor, one has to find a second compatible pure spinor, and calculate the RR flux by acting on the latter with $(\mathrm{d}-H \wedge)$. In order to promote a configuration satisfying the supersymmetry conditions to a full solution, one has to check the Bianchi Identities (BI) for all fluxes

$$
\begin{aligned}
\mathrm{d}(F) & =\delta(\text { source }), \\
\mathrm{d} H & =0 .
\end{aligned}
$$

Because of tadpole cancellation, the sources charged with respect to RR fields need to have an overall negative tension, and hence the dominant charge is that of an O-plane. This is the final step in the search for $\mathcal{N}=1$ vacua on Minkowski. For $A d S_{4}$ supersymmetric solution, a similar procedure can be defined.

For non-supersymmetric backgrounds, the situation is much more complicated, since, a priori, first order equations such as (1.1) are not anymore valid. Recently a procedure has been proposed in [11] that generalizes to non-supersymmetric backgrounds the first order pure spinor equations (1.1). The idea of [11] is to decompose the supersymmetry breaking terms in (1.1) in the $\operatorname{Spin}(6,6)$ basis constructed from the pure spinors. For instance, for Minkowski compactifications, the modified first order equations are

$$
\begin{aligned}
\mathrm{d}_{H}\left(e^{2 A-\phi} \Phi_{1}\right) & =\Upsilon, \\
\mathrm{d}_{H}\left(e^{A-\phi} \operatorname{Re} \Phi_{2}\right) & =\operatorname{Re} \Xi, \\
\mathrm{d}_{H}\left(e^{3 A-\phi} \operatorname{Im} \Phi_{2}\right)-\frac{|a|^{2}}{8} e^{3 A} * \lambda(F) & =\operatorname{Im} \Xi,
\end{aligned}
$$

where schematically

$$
\begin{aligned}
\Upsilon= & a_{0} \Phi_{2}+\widetilde{a}_{0} \bar{\Phi}_{2}+a_{m}^{1} \gamma^{m} \Phi_{1}+a_{m}^{2} \Phi_{1} \gamma^{m}+\widetilde{a}_{m}^{1} \gamma^{m} \bar{\Phi}_{1}+\widetilde{a}_{m}^{2} \bar{\Phi}_{1} \gamma^{m} \\
& +a_{m n} \gamma^{m} \Phi_{2} \gamma^{n}+\widetilde{a}_{m n} \gamma^{n} \bar{\Phi}_{2} \gamma^{m} \\
\Xi= & b_{0} \Phi_{1}+\widetilde{b}_{0} \bar{\Phi}_{1}+b_{m}^{1} \gamma^{m} \Phi_{2}+b_{m}^{2} \Phi_{2} \gamma^{m}+b_{m n} \gamma^{m} \Phi_{1} \gamma^{n}+\widetilde{b}_{m n} \gamma^{n} \bar{\Phi}_{1} \gamma^{m} .
\end{aligned}
$$

In the particular case of an $\mathrm{SU}(3)$ structure, this decomposition is equivalent to the expansion of (1.1) in the $\mathrm{SU}(3)$ torsion classes.

Equations (1.9) rely on the assumption that the four-dimensional space-time admits Killing spinors and that the supersymmetry breaking is due to the internal spinors only. This applies of course to Minkowski and Anti de Sitter backgrounds, but not for de Sitter solutions or cases when supersymmetry is broken in four-dimensions.

The purpose of the paper is twofold. On one side, we would like to make some first steps towards determining a set of first order equations also for configurations where fourdimensional supersymmetry is broken. In particular, we shall propose a first order equation 
similar to the last equation in (1.1), so that the flux equations of motion (1.7) follow automatically. On the other side, we would like to reexamine the problem of finding de Sitter vacua directly in ten-dimensions and focus only on simple conservative compactifications (i.e. "geometric" set-up).

We will consider de Sitter vacua in IIA supergravity. In this context, several no-go theorems and ways of circumventing them have been proposed [12-20]. In particular, in presence of O6/D6 sources, a minimal requirement to evade the no-go theorem [14] is to have a negatively curved internal manifold and a non-zero $F_{0}$ (Romans mass parameter) $[12,16,18]$. Therefore, we will focus on type IIA configurations with non-zero NS three-form and RR zero and two-forms. Moreover, we assume that all the sources (there may be intersecting ones) are space-time filling and are of the same dimension $p=6$.

Tracing the four-dimensional part of Einstein equation and using the dilaton equation of motion, one can show that the four-dimensional curvature and the "source term" can be written as

$$
\begin{aligned}
R_{4} & =\frac{2}{3}\left[-R_{6}-\frac{g_{s}^{2}}{2}\left|F_{2}\right|^{2}+\frac{1}{2}\left(|H|^{2}-g_{s}^{2}\left|F_{0}\right|^{2}\right)\right], \\
g_{s} \frac{T_{0}}{p+1} & =\frac{1}{3}\left[-2 R_{6}+|H|^{2}+2 g_{s}^{2}\left(\left|F_{0}\right|^{2}+\left|F_{2}\right|^{2}\right)\right],
\end{aligned}
$$

where, for simplicity, we have taken constant dilaton, $e^{\phi}=g_{s}$, and no-warping. ${ }^{3}$

Further simplifications are possible when one assumes that the sources preserve the supersymmetry of the bulk; this condition is usually expressed in terms of an equation involving the bulk supersymmetry parameters and the world-volume chiral operator entering the $\kappa$-symmetry transformations. Up to terms quadratic in the $\kappa$-symmetry condition, one can always rewrite the brane world-volume action as the pullback of the non-integrable pure spinor

$$
\left(i^{*}\left[\operatorname{Im} \Phi_{2}\right] \wedge e^{\mathcal{F}}\right)=\frac{|a|^{2}}{8} \sqrt{\left|i^{*}[g]+\mathcal{F}\right|} \mathrm{d}^{\Sigma} x
$$

where $i$ denotes the embedding of the world-volume into the internal manifold $M, g$ is the internal metric and $\mathcal{F}$ is the gauge invariant combination of the field strength of the worldvolume gauge field and the pullback of $B$. For sources preserving the supersymmetry of the bulk, one can then replace the DBI action by the left-hand side of (1.16). The equations of motion derived from both actions are the same, since the corrections would be linear in

\footnotetext{
${ }^{3}$ In general, with non-trivial dilaton and a ten-dimensional metric of the form

$$
d s^{2}=e^{2 A(y)} g_{\mu \nu}(x) \mathrm{d} x^{\mu} \mathrm{d} x^{\nu}+g_{m n}(y) \mathrm{d} y^{m} \mathrm{~d} y^{n},
$$

equation (1.12) becomes

$$
\begin{aligned}
e^{-2 A} R_{4}= & \frac{2}{3}\left[-R_{6}-\frac{e^{2 \phi}}{2}\left|F_{2}\right|^{2}+\frac{1}{2}\left(|H|^{2}-e^{2 \phi}\left|F_{0}\right|^{2}\right)\right] \\
& -8 \nabla^{2} A+20\left|\partial_{m} A\right|^{2}-\frac{8}{3} \nabla^{2} \phi+\frac{8}{3}\left|\partial_{m} \phi\right|^{2}-\frac{32}{3} g^{m n} \partial_{m} A \partial_{n} \phi
\end{aligned}
$$
}

All derivatives are taken with respect to the coordinates on $M$. We shall return to the discussion of the warp factor and the dilaton. 
the $\kappa$-symmetry condition, and then vanishing in the supersymmetric case. In particular, one can show that the world-volume equations of motion are then automatically implied by the last equation of (1.1). So the condition (1.16) together with the last equation of (1.1) give (generalized) calibrated sources, i.e. their energy density is minimized [6, 21-23].

For such supersymmetric configurations, the four- and six-dimensional traces of the source energy-momentum tensor and the source term in the dilaton equation are all proportional to each other, and one arrives at

$$
\begin{aligned}
& R_{4}=\frac{2}{3}\left(g_{s}^{2}\left|F_{0}\right|^{2}-|H|^{2}\right), \\
& R_{6}+\frac{1}{2} g_{s}^{2}\left|F_{2}\right|^{2}+\frac{3}{2}\left(g_{s}^{2}\left|F_{0}\right|^{2}-|H|^{2}\right)=0,
\end{aligned}
$$

together with eq. (1.13). The last equation is just a constraint on internal quantities, while the two others fix $R_{4}$ and the source term $T_{0}$. From these two equations we recover the minimal requirement of having $F_{0} \neq 0$ and $R_{6}<0$. In practice, however, this is not enough to find a de Sitter vacuum. In particular, we can see that $F_{0}$ alone can give a positive value to the cosmological constant, and adding more fluxes, $F_{4}$ and $F_{6}$, does not help since they give negative contributions. Indeed, up to date, all known examples of stable de Sitter vacua require some additional ingredients such as KK monopoles and Wilson lines [15], non-geometric fluxes [24, 25], or $\alpha^{\prime}$ corrections and probe D6 branes [26]. In this paper, we will work in ten dimensions and mainly focus on classical geometric compactifications.

Since we are interested in non-supersymmetric backgrounds, there is a priori no reason to impose that the sources preserve the bulk supersymmetry. The condition (1.16) could therefore be violated. To do so, we make in this paper the following proposal: we replace (1.16), in analogy with (1.9) where the violation of the bulk supersymmetry conditions is encoded in the general polyforms $\Upsilon$ and $\Xi$, by

$$
\left(i^{*}\left[\operatorname{Im} X_{-}\right] \wedge e^{\mathcal{F}}\right)=\sqrt{\left|i^{*}[g]+\mathcal{F}\right|} \mathrm{d}^{\Sigma} x
$$

where $X_{-}$is an odd polyform given by a general expansion similar to $\Upsilon$. For supersymmetric configurations, $X_{-}$reduces to $8 \Phi_{-}$, but in general, it is no longer a pure spinor. As discussed, such a replacement for sources preserving bulk supersymmetry is correct up to quadratic terms in the $\kappa$-symmetry condition, and corrections to the equations of motion derived from it will vanish linearly if the condition holds. In our case the structure of the corrections is not explicit, and we cannot conclude that the equations of motion derived from left-hand side of (1.19) are the same as those derived from DBI. We will thus proceed as follows: we first find solutions using the equations derived from the left-hand side of (1.19) and then we will check whether these are solutions to the equations derived from the standard DBI action.

An advantage of replacing DBI by the pullback of a form from the bulk is that it is actually easier to take the variation with respect to the various fields, in particular the bulk ones. Moreover the variation of the left-hand side of (1.19) with respect to the metric will lead to interesting consequences for de Sitter solutions: new terms are generated in the energy momentum tensor which help to lift the cosmological constant to positive 
values (see further in (1.24)). This is the main motivation for using this proposal, but a full understanding of it should be provided in future work. One possible interpretation is that such sources could be thought as standard D-branes or O-planes but their embedding into space-time (here into $M$ ) is modified. While for supersymmetric configurations the geometry of the subspace wrapped by the source is encoded in $\operatorname{Im} \Phi_{2}$, here it would be encoded in the more general expansion $\operatorname{Im} X_{-}$, of which $\operatorname{Im} \Phi_{2}$ is only one possible term. Therefore, the breaking of bulk supersymmetry seems to come from allowing more general geometries for the wrapped subspaces, and the new terms in the energy momentum tensor could come from the non standard embedding, in particular a dependence of the embedding functions on the metric moduli.

Since the bulk supersymmetry is broken, we could as well modify (1.1) and, in view of (1.19), we propose here the following generalization of the first order conditions:

$$
\begin{aligned}
& \mathrm{d}_{H}\left(e^{2 A-\phi} \operatorname{Re} X_{-}\right)=0, \\
& \mathrm{~d}_{H}\left(e^{4 A-\phi} \operatorname{Im} X_{-}\right)=c_{0} e^{4 A} * \lambda(F),
\end{aligned}
$$

where $c_{0}$ is a positive constant fixed by the parameters of the solution. Hence the introduction of $X_{-}$allows, as for the supersymmetric case, to trade the RR equations of motion for first order equations (clearly (1.7) follows by differentiating (1.21)), while, in addition, it helps via (1.19) to solve the internal Einstein equation. This is a first step towards developing a more systematic procedure to find non-supersymmetric backgrounds.

For the NSNS fields, we will check explicitly that our solution is a solution to the equations of motion derived from DBI, making use of a dependence of the embedding functions on the metric moduli. What remains are the world-volume fields (note the $\mathcal{F}$ will be trivial for us). Let us comment on their equations. As mentioned previously, for sources preserving bulk supersymmetry, a world-volume equation of motion, obtained by varying (1.16) augmented by the WZ terms, turns out to follow simply from a partial pullback of the bulk pure spinor equation (1.1). Then the minimization of the worldvolume energy is automatic [21, 22]. The equations of motion derived from the left-hand side of (1.19) should also be compared with the partial pullback of (1.21). We shall denote the transverse differentiation by $\partial_{\alpha}$ and a flux with all but one index pulled back to the world-volume by $i^{*}[F]_{\alpha}$. Neglecting the world-volume gauge fields, we can write the resulting equation as

$$
\partial_{\alpha}\left(i^{*}\left[e^{4 A-\phi} \operatorname{Im}\left(e^{-B} X_{-}\right)\right]\right)-i^{*}\left[e^{4 A} e^{-B} * \lambda(F)\right]_{\alpha}=0 .
$$

Comparison with the components of (1.21) gives

$$
\left(c_{0}-1\right) i^{*}\left[e^{-B} * \lambda(F)\right]_{\alpha}=0 .
$$

In the supersymmetric case, where we replace $X_{-}$by $\Phi_{-}, c_{0}=1$ and the equation is automatically satisfied. Here we will consider solutions with a vanishing partial pullback $i^{*}\left[e^{-B} * \lambda(F)\right]_{\alpha}$, so the world-volume equations derived from the left-hand side of (1.19) will be satisfied, making the energy of our sources extremized. We will also check that our solution satisfies the equations of motion obtained by the variation of the standard $\mathrm{DBI}+\mathrm{WZ}$ action. 
The strategy to find a non-supersymmetric solution to our proposed action is the following. We start with one particular solution to (1.1), which is a supersymmetric compactification of IIA on a solvmanifold labeled $s 2.5$. The solvable algebra is given by $\left(q_{1} 35, q_{2} 45,-q_{2} 15,-q_{1} 25,0,0\right)$. The solution involves intersecting O6 planes (and possibly D6 branes - depending on the choice of parameters). Due to the general problems in constructing localized intersecting branes, the sources are smeared, and hence the model would suffer from general criticism [27]. It does have some convenient features though, and it serves as a good illustration to the method we would like to propose. We shall return to the question of localization and to the possibility of incorporating warp factor and non-constant dilaton in the paper. For now, without further apologies, we shall use the $s 2.5$ model as a point of departure for our non-supersymmetric construction.

$s 2.5$ is a special case of a more general solvable algebra $\left(q_{1}(p 25+35), q_{2}(p 15+\right.$ $\left.45), q_{2}(p 45-15), q_{1}(p 35-25), 0,0\right)$. It is then natural to see whether the corresponding solvmanifold also admits solutions. A natural ansatz would be generalizations of the supersymmetric solutions on $s 2.5$. To do so, we shall extend to solvmanifolds the twist transformation worked out in [28] for nilmanifolds. It turns out that the first two equations of (1.1) can be satisfied for $p \neq 0$ provided a certain combination of moduli, which we call $\lambda$, takes value 1 . In other words, for generic $p$ and $\lambda=1$ we find supersymmetric solutions (corresponding to a vanishing four-dimensional curvature). For generic $\lambda$, the pure spinor equations are not satisfied and supersymmetry is broken. It is certainly of great practical importance to have a convenient limit in which our construction can be tested.

The proposed source action (1.19) allows to rewrite (1.17) for the four dimensional Ricci tensor as

$$
R_{4}=\frac{2}{3}\left(\frac{g_{s}}{2}\left(T_{0}-T\right)+g_{s}^{2}\left|F_{0}\right|^{2}-|H|^{2}\right),
$$

where the source term $T_{0}$ is different from the trace of the energy momentum tensor $T$. As can be seen from (1.13), $T_{0}$ gives a positive contribution to $R_{4}$ and in our case, it turns out that $T_{0}-T$ is also positive. Thus, with our proposal (1.19) we are indeed able to find a ten-dimensional de Sitter solution. Checking that it also satisfies the equations of motion derived from the standard source action (with a dependence of the embedding functions on the metric moduli) will make it a solution of type IIA supergravity.

The details of the solution, as well as the treatment of our proposal for supersymmetry breaking branes, are presented in section 3. This discussion is complemented by the analysis of the four-dimensional effective potential. In particular, we will discuss how the supersymmetry breaking proposal for the sources provides new terms in the potential. Also we will perform an analysis of stability of the solution in the volume and dilaton moduli.

While our discussion for de Sitter solution is based on a specific example, the construction is more general, and we present much of the technical machinery in section 2 . This contains a discussion of supersymmetric solutions, and the twist construction of solvmanifolds which serve as internal spaces. The construction has been used previously for nilmanifolds (which are an iteration of torus bundles over a base manifold being a torus itself) [28]. It is extended here to the case of solvable algebras. Our basic example is based on a solvable group that admits a lattice and hence yields a compact six-dimensional solv- 
manifold. As we shall see the construction can be applied also to algebras that admit no such lattice, and it may lead to non-geometric backgrounds. A more formal presentation of the solvable algebras and the geometry of (compact) solvmanifolds is given in appendix A. In appendix B we discuss some global aspects of T-duality on solvmanifolds.

\section{Supersymmetric backgrounds, solvmanifolds and twist transforma- tions}

In this paper we are interested in string backgrounds where the internal compactification manifold is a solvmanifold. Nil- and solvmanifolds have been extensively used in type II compactifications, both to four-dimensional Minkowski or Anti de Sitter, and appear to be good candidates for possible de Sitter vacua as well. Indeed their geometry is pretty well understood (for instance all nilmanifolds are generalized Calabi-Yaus [29]) and, in particular, they can have negative curvature and therefore support internal fluxes (as well as D-branes and O-plane sources).

Nil- and solvmanifolds are homogeneous spaces constructed from nilpotent or solvable groups $G$, nilpotent being actually a particular case of solvable. When the group $G$ is not compact, the manifold can be made compact by quotienting $G$ by a lattice $\Gamma$, i.e. a discrete co-compact subgroup of $G$. The dimension of the resulting manifold ${ }^{4}$ is the same as that of the group $G$. In this paper we will focus on manifolds of dimension six. It can be proven [30] that a lattice $\Gamma$ can always be found for nilmanifolds, while for solvmanifolds its existence is harder to establish. We refer to appendix A for a detailed discussion of the algebraic aspects and the compactness properties of nil and solvmanifolds. Here, we focus on their geometry.

Given a $d$-dimensional Lie algebra $\mathfrak{g}$ expressed in some vector basis $\left\{E_{1}, \ldots, E_{d}\right\}$ as

$$
\left[E_{b}, E_{c}\right]=f_{b c}^{a} E_{a}
$$

where $f_{b c}^{a}$ are the structure constants, we can define the dual space of one-forms $\mathfrak{g}^{*}$ with basis $\left\{e^{1}, \ldots, e^{d}\right\}$. They satisfy the Maurer-Cartan equation

$$
\mathrm{d} e^{a}=-\frac{1}{2} f_{b c}^{a} e^{b} \wedge e^{c}=-\sum_{b<c} f_{b c}^{a} e^{b} \wedge e^{c}
$$

with the exterior derivative d. Since $\mathfrak{g}^{*} \approx T_{e} G^{*},\left\{e^{1}, \ldots, e^{d}\right\}$ provide, by left invariance, a basis for the cotangent space $T_{x} G^{*}$ at every point $x \in G$ and, thus, are globally defined one-forms on the manifold. When the manifold is obtained as a quotient with a lattice $\Gamma$, the one-forms will have non trivial identification through the lattice action. ${ }^{5} \mathrm{Nil}$ and

\footnotetext{
${ }^{4}$ This definition of solvmanifold it is not the most general: one could consider cases where the $d$ dimensional solvmanifold is the quotient of a higher dimensional group with a continuous subgroup $\Gamma$. This is the case for the Klein bottle, for instance.

${ }^{5}$ In general there is a natural inclusion $\left(\Lambda \mathfrak{g}^{*}, \delta\right) \rightarrow(\Lambda(G / \Gamma), d)$ between the Chevalley-Eilenberg complex on $G$ and the de Rham complex of differential forms on $G / \Gamma$. This inclusion induces an injection map between cohomology groups $H^{*}(\mathfrak{g}) \rightarrow H_{d R}^{*}(G / \Gamma)$ which turns out to be an isomorphism for completely solvable groups. We recall that a Lie group $G$ with Lie algebra $\mathfrak{g}$ is said to be completely solvable if the
} 
solvmanifolds, as we define them in this paper, are always parallelizable [32], even if they are not necessarily Lie groups.

The Maurer-Cartan equations reflect the topological structure of the corresponding manifolds. For example, nilmanifolds all consist of iterated fibrations of circles over tori, where the iterated structure is related to the descending or ascending series of the algebra (see $[29,33,34])$. This can be easily seen on a very simple example, the nilmanifold obtained from the three-dimensional Heisenberg algebra

$$
\left[E_{2}, E_{3}\right]=E_{1} \quad \Leftrightarrow \quad \mathrm{d} e^{1}=-e^{2} \wedge e^{3} .
$$

The Maurer-Cartan equation is solved by the one-forms

$$
e^{1}=\mathrm{d} x^{1}-x^{2} \mathrm{~d} x^{3}, e^{2}=\mathrm{d} x^{2}, e^{3}=\mathrm{d} x^{3} .
$$

From the connection form, $-x^{2} \mathrm{~d} x^{3}$, one can read the topology of the nilmanifold in question, which is a non-trivial fibration of the circle in direction 1 on the two-torus in directions 2,3 :

$$
\begin{gathered}
S_{\{1\}}^{1} \hookrightarrow H / \Gamma_{1} \\
\downarrow \\
\\
\\
T_{\{23\}}^{2}
\end{gathered}
$$

Solvmanifolds are classified according to the dimension of the nilradical $\mathfrak{n}$ (the largest nilpotent ideal) of the corresponding algebra. In six dimensions, $\mathfrak{n}$ can have dimension from 3 to 6 . If $\operatorname{dim} \mathfrak{n}=6$, then $\mathfrak{n}=\mathfrak{g}$ and the algebra is nilpotent. At the level of the group ${ }^{6}$ we have that, if $\operatorname{dim} N<6$, then $G$ contains an abelian subgroup of dimension $k[35,36]$. This means we have $G / N=\mathbb{R}^{k}$. When the group admits a lattice $\Gamma$, one can show that $\Gamma_{N}=\Gamma \cap N$ is a lattice in $N, \Gamma N=N \Gamma$ is a closed subgroup of $G$, and so $G /(N \Gamma)=T^{k}$ is a torus. The solvmanifold is a non-trivial fibration of a nilmanifold over the torus $T^{k}$

$$
\begin{array}{ccc}
N / \Gamma_{N}=(N \Gamma) / \Gamma \hookrightarrow & G / \Gamma \\
& \downarrow \\
& T^{k}=G /(N \Gamma)
\end{array}
$$

This bundle is called the Mostow bundle [37]. As we shall see, the corresponding fibration can be more complicated than in the nilmanifold case. In general, Mostow bundles are not principal.

In the following we will restrict to almost abelian solvable groups, for which the construction of the Mostow bundle is particularly simple. Consider first almost nilpotent solvable groups. These are solvable groups that have nilradical of $\operatorname{dimension} \operatorname{dim} N=\operatorname{dim} G-1$. As discussed in appendix A.1, the group is then given by the semi-direct product

$$
G=\mathbb{R} \ltimes_{\mu} N
$$

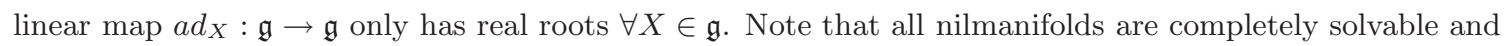
thus the injection is an isomorphism (Nomizu's theorem [31]), the extension to non-nilpotent completely solvable groups being the so-called Hattori theorem [32]. For more details and for a list of Betti numbers of solvmanifolds up to dimension six see [33].

${ }^{6}$ We denote by $\mathfrak{n}$ the ideal in the algebra and with $N$ the corresponding subgroup. 
of its nilradical with $\mathbb{R}$, where $\mu$ is some action on $N$ depending on the direction $\mathbb{R}$

$$
\left(t_{1}, n_{1}\right) \cdot\left(t_{2}, n_{2}\right)=\left(t_{1} \cdot t_{2}, n_{1} \cdot \mu_{t_{1}}\left(n_{2}\right)\right) \quad \forall(t, n) \in \mathbb{R} \times N .
$$

In general, we label by $t$ the coordinate on $\mathbb{R}$ and by $\partial_{t}$ the corresponding vector of the algebra. From a geometrical point of view, $\mu(t)$ encodes the fibration of the Mostow bundle.

An almost abelian solvable group is an almost nilpotent group whose nilradical is abelian

$$
N=\mathbb{R}^{\operatorname{dim} G-1}
$$

In this case, the action of $\mathbb{R}$ on $N$ is given by

$$
\mu(t)=A d_{\partial_{t}}(\mathfrak{n})=e^{t a d_{\partial_{t}}(\mathfrak{n})} .
$$

Another nice feature of almost abelian solvable groups is that a simple criterion exists to determine whether the associated solvmanifold is compact: the group admits a lattice if and only if there exists a $t_{0} \neq 0$ for which $\mu\left(t_{0}\right)$ can be conjugated to an integer matrix.

As an example, we can consider two three-dimensional almost abelian solvable algebras

$$
\begin{aligned}
& \varepsilon_{2}:\left[E_{2}, E_{3}\right]=E_{1} \quad \Leftrightarrow \quad \mathrm{d} e^{1}=-e^{2} \wedge e^{3} \\
& {\left[E_{1}, E_{3}\right]=-E_{2} \quad \Leftrightarrow \quad \mathrm{d} e^{2}=e^{1} \wedge e^{3}} \\
& \varepsilon_{1,1}:\left[E_{1}, E_{3}\right]=E_{1} \quad \Leftrightarrow \quad \mathrm{d} e^{1}=-e^{1} \wedge e^{3} \\
& {\left[E_{2}, E_{3}\right]=-E_{2} \quad \Leftrightarrow \quad \mathrm{d} e^{2}=e^{2} \wedge e^{3} .}
\end{aligned}
$$

In the following, we will label the algebras according to their Maurer-Cartan equations. For instance, $\varepsilon_{2}$ is denoted by $(-23,13,0)$, where each entry $i$ gives the result of $\mathrm{d} e^{i}$.

For the algebra $\varepsilon_{2}:(-23,13,0)$, the nilradical is given by $\mathfrak{n}=\left\{E_{1}, E_{2}\right\}$ and $\partial_{t}=E_{3}$. In this basis, the restriction of the adjoint representation to the nilradical is

$$
a d_{\partial_{t}}(\mathfrak{n})=\left(\begin{array}{cc}
0 & -1 \\
1 & 0
\end{array}\right)
$$

which gives a $\mu$ matrix of the form

$$
\mu(t)=e^{t a d_{\partial_{t}}(\mathfrak{n})}=\left(\begin{array}{cc}
\cos (t) & -\sin (t) \\
\sin (t) & \cos (t)
\end{array}\right) .
$$

It is easy to see that, for $t_{0}=n \frac{\pi}{2}$, with $n \in \mathbb{Z}^{*}, \mu\left(t_{0}\right)$ is an integer matrix and hence the corresponding manifold is compact.

For the algebra $\varepsilon_{1,1}:(-13,23,0)$ the analysis is less straightforward. The nilradical is $\mathfrak{n}=\left\{E_{1}, E_{2}\right\}$ and again $\partial_{t}=E_{3}$. Then, in the $\left(E_{1}, E_{2}\right)$ basis,

$$
a d_{\partial_{t}}(\mathfrak{n})=\left(\begin{array}{cc}
-1 & 0 \\
0 & 1
\end{array}\right), \mu(t)=e^{t a d_{\partial_{t}}(\mathfrak{n})}=\left(\begin{array}{cc}
e^{-t} & 0 \\
0 & e^{t}
\end{array}\right),
$$




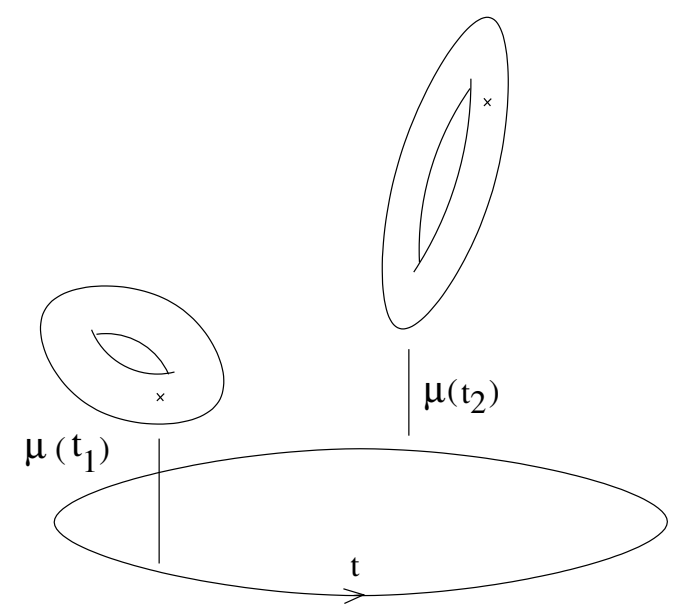

Figure 1. Mostow bundle for the solvmanifolds $\epsilon_{2}$ and $\epsilon_{1,1}$. The base is the circle in the $t$ direction, and due to the nilradical being abelian the fiber is $T^{2}$. The fibration is encoded in $\mu(t)$ which is either a rotation or a "hyperbolic rotation" twisting the $T^{2}$ moving along the base.

and it is clearly not possible to find a $t_{0} \neq 0$ such that $\mu\left(t_{0}\right)$ is an integer. To see whether the group admits a lattice, we then have to go to another basis. In other words, $\mu\left(t_{0}\right)$ will be conjugated to an integer matrix. As in [16], we can define a new basis

$$
E_{1} \rightarrow \sqrt{\frac{q_{2}}{q_{1}}} \frac{E_{1}-E_{2}}{\sqrt{2}}, E_{2} \rightarrow \frac{E_{1}+E_{2}}{\sqrt{2}}, E_{3} \rightarrow \sqrt{q_{1} q_{2}} E_{3},
$$

with $q_{1}, q_{2}$ strictly positive constants, such that the algebra reads

$$
\left[E_{1}, E_{3}\right]=q_{2} E_{2} \quad\left[E_{2}, E_{3}\right]=q_{1} E_{1} .
$$

In this new basis

$$
a d_{\partial_{t}}(\mathfrak{n})=\left(\begin{array}{cc}
0 & -q_{1} \\
-q_{2} & 0
\end{array}\right), \mu(t)=\left(\begin{array}{cc}
\cosh \left(\sqrt{q_{1} q_{2}} t\right) & -\sqrt{\frac{q_{1}}{q_{2}}} \sinh \left(\sqrt{q_{1} q_{2}} t\right) \\
-\sqrt{\frac{q_{2}}{q_{1}}} \sinh \left(\sqrt{q_{1} q_{2}} t\right) & \cosh \left(\sqrt{q_{1} q_{2}} t\right)
\end{array}\right),
$$

so that $\mu(t)$ can be made integer with the choice of parameters

$$
t_{0} \neq 0, \cosh \left(\sqrt{q_{1} q_{2}} t_{0}\right)=n_{1}, \frac{q_{1}}{q_{2}}=\frac{n_{2}}{n_{3}}, n_{2} n_{3}=n_{1}^{2}-1, n_{1,2,3} \in \mathbb{Z}^{*} .
$$

Thus also the algebra $\varepsilon_{1,1}$ can be used to construct compact solvmanifolds (see figure 1 for illustration). Notice that the values $q_{1}=q_{2}=1$ are not allowed by the integer condition (2.19).

\subsection{Twist construction of (almost abelian) solvmanifolds}

In the previous section we showed how to obtain explicitly, at least for almost abelian solvmanifolds, the operator $\mu(t)$ giving the structure of the Mostow bundle and what condition it has to satisfy in order for the manifold to be compact. 
In this section we focus on six-dimensional almost abelian algebras and the corresponding compact solvmanifolds, and we discuss how to use the adjoint action $\mu(t)$ to construct the globally defined one-forms of the solvmanifolds from those on $T^{6}$. In appendix A.3 we show how the construction works in general for almost nilpotent and nilpotent algebras. For nilmanifolds the construction proposed in [28] is recovered.

Let us first discard global issues related to the compactness of the manifolds. Then, given an almost abelian solvable group $G$, we want to relate one-forms on $T^{*} \mathbb{R}^{6}$ to those of $T^{*} G=\mathfrak{g}^{*}$

$$
A\left(\begin{array}{c}
\mathrm{d} x^{1} \\
\vdots \\
\mathrm{d} x^{6}
\end{array}\right)=\left(\begin{array}{c}
e^{1} \\
\vdots \\
e^{6}
\end{array}\right)
$$

Here $A$ is a local matrix that should contain the bundle structure of $G$. From the Mostow bundle, (2.6), it is natural to identify $x^{6}$ with the coordinate $t$ parametrising the $\mathbb{R}$ subalgebra and to take the corresponding one-form as $\mathrm{d} x^{6}=\mathrm{d} t$. Then the matrix $A$ takes the form

$$
A=\left(\begin{array}{cc}
A_{M} & 0 \\
0 & 1
\end{array}\right),
$$

where $A_{M}$ is a five-dimensional matrix given by

$$
A_{M}=\mu(-t)=\mu(t)^{-1}=e^{-t a d_{\partial_{t}}(\mathfrak{n})} .
$$

It is straightforward to show that the forms constructed this way verify the Maurer-Cartan equation (see (A.25)):

$$
\mathrm{d} e^{i}=\mathrm{d}\left(e^{-t a d_{\partial_{t}}}\right)_{k}{ }_{k} \wedge \mathrm{d} x^{k}=\cdots=-f^{i}{ }_{t j} \mathrm{~d} t \wedge e^{j} .
$$

Note that taking, for instance, $\mu(t)$ as in (2.14), the corresponding $A$ is not a diffeomorphism and therefore can change topology.

We now come back to the consistency of this construction and the question of compactness. To this end we need to investigate the monodromy properties of the matrix $A_{M}$ and the related one-forms under a complete turn around the base circle.

Let us consider the following identification: $t \sim t+t_{0}$ where $t_{0}$ is the periodicity of the base circle. To obtain a consistent construction (having globally defined one-forms) we must preserve the structure of the torus we are fibering over the $t$ direction. This amounts to asking that an arbitrary point of the torus is sent to an equivalent one after we come back to the point $t$ from which we started. The monodromies of the fiber are fixed, thus the only allowed shifts are given by their integer multiples. The way points in the torus are transformed when we go around the base circle is encoded in a matrix $M_{\mathcal{F}}$ which has to be integer valued. The identification along the $t$ direction is given by

$$
T_{6}:\left\{\begin{array}{l}
t \rightarrow t+t_{0} \\
x^{i} \rightarrow\left(M_{\mathcal{F}}\right)^{i}{ }_{j} x^{j}
\end{array} \quad i, j=1, \ldots, 5,\right.
$$


while those along the remaining directions are trivial

$$
T_{i}:\left\{\begin{array}{l}
x^{i} \rightarrow x^{i}+1 \\
x^{j} \rightarrow x^{j} \\
t \rightarrow t
\end{array} \quad i, j=1, \ldots, 5 ; i \neq j .\right.
$$

Let us now consider the one-forms (2.20) we have constructed via the twist $A_{M}$. It is straightforward to see that (2.20) are invariant under the trivial identifications, while under the non-trivial $T_{6}$, we have for $i, j=1, \ldots, 5$

$$
\tilde{e}^{i}=A_{M}\left(t+t_{0}\right)^{i}{ }_{j} \mathrm{~d} \tilde{x}^{j}=\left[A_{M}(t) A_{M}\left(t_{0}\right) M_{\mathcal{F}}\right]^{i}{ }_{j} \mathrm{~d} x^{j}
$$

The one-forms are globally defined if they are invariant under this identification:

$$
\tilde{e}^{i}=e^{i}=A_{M}(t)^{i}{ }_{j} \mathrm{~d} x^{j}
$$

Therefore, in the construction, we have to satisfy the following condition:

$$
A_{M}\left(t_{0}\right) M_{\mathcal{F}}=\mathbb{I}_{5} \Leftrightarrow M_{\mathcal{F}}=A_{M}^{-1}\left(t_{0}\right)=A_{M}\left(-t_{0}\right)
$$

Consistency requires the matrix $A_{M}$ to be such that $A_{M}\left(-t_{0}\right)$ is integer valued for at least one $t_{0} \neq 0$. This will impose a quantization condition on the period of the base circle, which can take only a discrete set of values (in general it will be a numerable set, as we will see in the examples). Once we fix $t_{0}$, the integer entries of $A_{M}\left(-t_{0}\right)$ will provide the set of identifications.

It is worth stressing that being able to give the correct identifications of the one-forms of the manifold is the same as having a lattice: the identifications (2.28) express the lattice action, and give globally defined one-forms only if $A_{M}\left(-t_{0}\right)=\mu\left(t_{0}\right)$ is integer valued for some $t_{0}$. As already discussed, this is the condition to have a lattice (as stated in [33], see also appendix A.2). Let us emphasize that the one-forms (2.20), constructed via the twist, are globally defined only if we start from a basis of the Lie algebra where $A_{M}(t)$ is integer valued for some value of $t$. We give a list of algebras in such a basis in appendix A.4.

Note that obtaining a set of globally defined one-forms is an expected result, since we are transforming a six-torus into a solvmanifold, which we know to be parallelizable. Moreover, we also know that, with a consistent twist, we are not leaving the geometrical framework.

As an example, we write the explicit form of the twist matrix for the two almost abelian six-dimensional algebras we need in this paper. ${ }^{7}$ In the basis where the one-forms are globally defined the two algebras are

$$
\begin{aligned}
& \mathfrak{g}_{5.7}^{1,-1,-1} \oplus \mathbb{R}:\left(q_{1} 25, q_{2} 15, q_{2} 45, q_{1} 35,0,0\right), \\
& \mathfrak{g}_{5.17}^{p,-p, \pm 1} \oplus \mathbb{R}:\left(q_{1}(p 25+35), q_{2}(p 15+45), q_{2}(p 45-15), q_{1}(p 35-25), 0,0\right) .
\end{aligned}
$$

\footnotetext{
${ }^{7}$ We use the same notation as in the standard classification of solvable algebras [33, 38, 39]: the number 5 indicates the dimension of the (indecomposable) algebra, while the second simply gives its position in the list of indecomposable algebras of dimension 5 .
} 
In both cases the parameters $q_{1}$ and $q_{2}$ are strictly positive. This is not the most general form of these algebras, which in general ${ }^{8}$ contain some free parameters $p, q$ and $r$. Here we wrote the values of the parameters for which we were able to find a lattice: $p=-q=-r=1$ for the first algebra and $r= \pm 1$ for the second.

In the rest of the paper, by abuse of notation, we will denote the algebra and the corresponding solvmanifold with the same name.

For (2.29), a type IIA solution with O6 planes was found in [40]. The algebra being a direct product of a trivial direction and a five-dimensional indecomposable algebra, the adjoint matrix $a d_{\partial_{x^{5}}}(\mathfrak{n})$ is block-diagonal, with the non-trivial blocks given by $-a d_{\partial_{t}}(\mathfrak{n})$ in (2.18) and its transpose. Then the twist matrix is

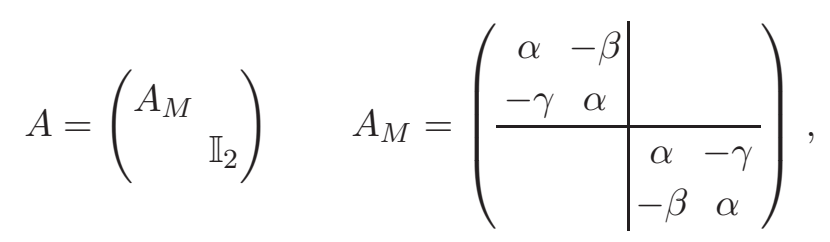

where, not to clutter notation, we defined

$$
\begin{aligned}
& \alpha=\cosh \left(\sqrt{q_{1} q_{2}} x^{5}\right), \\
& \beta=\sqrt{\frac{q_{1}}{q_{2}}} \sinh \left(\sqrt{q_{1} q_{2}} x^{5}\right), \\
& \gamma=\sqrt{\frac{q_{2}}{q_{1}}} \sinh \left(\sqrt{q_{1} q_{2}} x^{5}\right) .
\end{aligned}
$$

The forms obtained by the twist (2.31) are globally defined [16]. Indeed they are invariant under constant shifts of each $x^{i}$ for $i=1,2,3,4$ and 6 , with the other variables fixed, and the following non-trivial identification under shifts for $x^{5}$

$$
\left(x^{1}, \ldots, x^{6}\right)=\left(\alpha x^{1}+\beta x^{2}, \gamma x^{1}+\alpha x^{2}, \alpha x^{3}+\gamma x^{4}, \beta x^{3}+\alpha x^{4}, x^{5}+l, x^{6}\right),
$$

where in $\alpha, \beta, \gamma$ we took $x^{5}=l$. For the above identifications to be discrete [16] $\alpha, \beta$, and $\gamma$ must be all integers. This is equivalent to having the matrix $\mu\left(x^{5}=l\right)$ integer and, hence, it is the same as the compactness criterion. The existence of a lattice for the solution in [40] was also discussed in [41]. In that case the parameters $\alpha, \beta$ and $\gamma$ were set to $\alpha=2, \beta=3, \gamma=1$.

For the second algebra, $\mathfrak{g}_{5.17}^{p,-p, r} \oplus \mathbb{R}$, we will consider separately the cases $p=0$ and $p \neq 0$. For $p=0$ it reduces to $\left(q_{1} 35, q_{2} 45,-q_{2} 15,-q_{1} 25,0,0\right)$ with $r^{2}=1$. This algebra

\footnotetext{
${ }^{8}$ The general form for $\mathfrak{g}_{5.7}^{p, q, r}$ is

$\frac{1}{2}\left(-\beta(1+r) 15+q_{1}(1-r) 25,-\beta(1+r) 25+q_{2}(1-r) 15,-\beta(q+p) 35+q_{2}(p-q) 45,-\beta(q+p) 45+q_{1}(p-q) 35,0\right)$,

where we set $\beta=\sqrt{q_{1} q_{2}}$. Similarly, for $\mathfrak{g}_{5.17}^{p,-p, r}$ we have

$\left(q_{1} p 25+\frac{1}{2}\left[q_{1}\left(r^{2}+1\right) 35+\beta\left(r^{2}-1\right) 45\right], q_{2} p 15+\frac{1}{2}\left[q_{2}\left(r^{2}+1\right) 45+\beta\left(r^{2}-1\right) 35\right], q_{2}(-15+p 45), q_{1}(-25+p 35), 0\right)$.
} 
and the associated manifold have been already considered in [41], where it was called $s 2.5$. For $p \neq 0$ the algebra can be seen as the direct sum

$$
\mathfrak{g}_{5.17}^{p,-p, r} \oplus \mathbb{R} \approx s 2.5+p\left(\mathfrak{g}_{5.7}^{1,-1,-1} \oplus \mathbb{R}\right) .
$$

The twist matrix is given by

$$
A=\left(\begin{array}{lll}
A_{1} A_{2} & \\
& & \mathbb{I}_{2}
\end{array}\right) .
$$

The two matrices $A_{1}$ and $A_{2}$ commute and give the two parts of the algebra

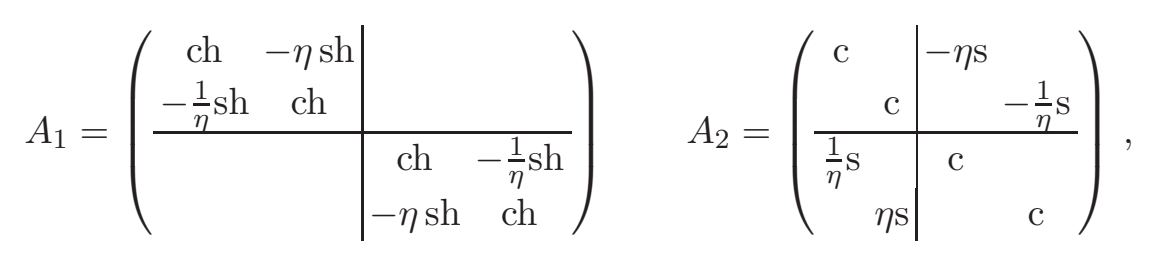

where now we define $\eta=\sqrt{\frac{q_{1}}{q_{2}}}$ and

$$
\begin{array}{ll}
\mathrm{ch}=\cosh \left(p \sqrt{q_{1} q_{2}} x^{5}\right) & \mathrm{c}=\cos \left(\sqrt{q_{1} q_{2}} x^{5}\right) \\
\mathrm{sh}=\sinh \left(p \sqrt{q_{1} q_{2}} x^{5}\right) & \mathrm{s}=\sin \left(\sqrt{q_{1} q_{2}} x^{5}\right) .
\end{array}
$$

In this case, imposing that the forms given by the twist (2.35) are globally defined under discrete identifications fixes the parameters in the twist to (with $x^{5}=l$ )

$$
\begin{aligned}
& \operatorname{ch~} \mathrm{c}=n_{1}, \eta \operatorname{sh~} \mathrm{c}=n_{2}, \frac{1}{\eta} \operatorname{sh~} \mathrm{c}=n_{3} \\
& \operatorname{sh~} \mathrm{s}=n_{4}, \eta \operatorname{ch~} \mathrm{s}=n_{5}, \frac{1}{\eta} \operatorname{ch~} \mathrm{s}=n_{6}, \quad n_{i} \in \mathbb{Z} .
\end{aligned}
$$

The equations above have no solutions if the integers $n_{i}$ are all non-zero. The only possibilities are either $n_{1}=n_{2}=n_{3}=0$ or $n_{4}=n_{5}=n_{6}=0$ (plus the case where all are zero, which is of no interest here). If one also imposes that the constraints must be solved both for $p=0$ and $p \neq 0$, the first option, $n_{1}=n_{2}=n_{3}=0$, has to be discarded and the only solution is

$$
\begin{aligned}
& n_{4}=n_{5}=n_{6}=0, s=0, l=\frac{k \pi}{\sqrt{q_{1} q_{2}}}, c=(-1)^{k}, \tilde{n}_{1}=(-1)^{k} n_{1}>0, k \in \mathbb{Z} \\
& \mathrm{ch}=\tilde{n}_{1}, \operatorname{sh}^{2}=n_{2} n_{3}, n_{3} \eta^{2}=n_{2}, n_{2} n_{3}=\tilde{n}_{1}^{2}-1, \quad p=\frac{\cosh ^{-1}\left(\tilde{n}_{1}\right)}{k \pi}
\end{aligned}
$$

$p$ is quantized by two integers, but one can show that it can be as close as we want to any real value (the ensemble is dense in $\mathbb{R}$ ).

\subsection{Twist transformations in generalized geometry}

The twist defined in the previous section has a natural embedding in generalized geometry. The basic idea of generalized geometry is to combine the tangent and cotangent bundle of a given manifold $M$ (here the internal manifold of our compactification) into a single 
object, the generalized tangent bundle $E$. This is an extension of $T M$ by $T^{*} M$. Locally a section of $E$ is a sum of a vector and a one-form

$$
X=v+\xi \in T M \oplus T^{*} M,
$$

which is glued on the overlap of two local patches, $U_{\alpha}$ and $U_{\beta}$, by

$$
\left(\begin{array}{l}
v \\
\xi
\end{array}\right)_{(\alpha)}=\left(\begin{array}{cc}
a & 0 \\
\omega a & a^{-T}
\end{array}\right)_{(\alpha \beta)}\left(\begin{array}{l}
v \\
\xi
\end{array}\right)_{(\beta)} .
$$

$a$ is an element of $\operatorname{GL}(d, \mathbb{R})$, and gives the usual gluing of vectors and one-forms $\left(a^{-T}=\right.$ $\left(a^{-1}\right)^{T}$ ), while $\omega$ is a two-form such that $\omega_{(\alpha \beta)}=-\mathrm{d} \Lambda_{(\alpha \beta)}$. $\omega$ is related to the non-trivial fibration of $T^{*} M$ over $T M$ : this is encoded in a local two-form (the "connective structure" of a gerbe) that is interpreted as the $B$-field, and $\omega$ corresponds to its gauge transformation.

For the backgrounds we will consider in this paper, $B=0$, meaning that the generalized tangent bundle is trivial and can be identified with $T M \oplus T^{*} M$.

$E$ is endowed with two metrics

$$
\mathcal{I}=\left(\begin{array}{ll}
0 & \mathbb{I} \\
\mathbb{I} & 0
\end{array}\right) \quad \mathcal{H}=\left(\begin{array}{cc}
g-B g^{-1} g & B g^{-1} \\
-g^{-1} B & g^{-1}
\end{array}\right)
$$

where $\mathcal{I}$ is the natural metric on $E$ (which is used to derive the Clifford algebra) while the generalized metric $\mathcal{H}$ encodes the information about the metric and the $B$-field of the background.

The metric $\mathcal{I}$ is invariant under $\mathrm{O}(d, d)$ transformations, which can be parametrised by $\operatorname{Gl}(d)$ transformations

$$
X=v+\xi \mapsto X^{\prime}=A v+A^{-T} \xi,
$$

plus shifts by a two-form $b$ and a two-vector $\beta$

$$
\begin{aligned}
& X=v+\xi \mapsto X^{\prime}=v+\left(\xi-i_{v} b\right), \\
& X=v+\xi \mapsto X^{\prime}=(v+\beta \cdot \xi)+\xi .
\end{aligned}
$$

These are the so-called $B$ - and $\beta$-transforms.

On $E$ one can define generalized vielbeine $\mathcal{E}$, such that

$$
\mathcal{I}=\mathcal{E}^{T}\left(\begin{array}{ll}
0 & \mathbb{I} \\
\mathbb{I} & 0
\end{array}\right) \mathcal{E} \quad \mathcal{H}=\mathcal{E}^{T}\left(\begin{array}{ll}
\mathbb{I} & 0 \\
0 & \mathbb{I}
\end{array}\right) \mathcal{E} .
$$

Explicitly, the generalized vielbeine can be put in the form

$$
\mathcal{E}^{A}{ }_{M}=\left(\begin{array}{cc}
e^{a}{ }_{m} & 0 \\
-(\hat{e} B)_{a m} & \hat{e}_{a}{ }^{m}
\end{array}\right),
$$

where $e^{a}{ }_{m}$ are the vielbeine on $M, \hat{e}=\left(e^{T}\right)^{-1}$, and $B$ is the $B$-field. Comparing the $\mathrm{O}(d, d)$ action on $\mathcal{E}$

$$
\mathcal{E} \mapsto \mathcal{E}^{\prime}=\mathcal{E} O=\left(\begin{array}{cc}
e^{a}{ }_{m} & 0 \\
-(\hat{e} B)_{a m} & \hat{e}_{a}{ }^{m}
\end{array}\right)\left(\begin{array}{cc}
A_{n}^{m} & B^{m n} \\
C_{m n} & D_{m}^{n}
\end{array}\right),
$$


with (2.20), it is natural to embed the twist transformation as

$$
O_{\mathrm{tw}}=\left(\begin{array}{cc}
A & 0 \\
0 & \left(A^{T}\right)^{-1}
\end{array}\right) .
$$

The polyforms $\Phi_{ \pm}$appearing in the supersymmetry conditions (1.1), correspond to ground states of the Clifford algebra $\operatorname{Cliff}(d, d)$, on $T M \oplus T^{*} M$. More precisely they are Majorana-Weyl $\operatorname{Spin}(d, d)$ spinors, the positive (negative) chirality corresponding to the even (odd) polyform. We will follow the conventions of [28]. We focus on manifolds of dimension six and construct $\mathrm{O}(6,6)$ bispinors in the Killing spinors on $M, \eta^{1,2}$,

$$
\Phi_{ \pm}=\eta_{+}^{1} \otimes \eta_{ \pm}^{2 \dagger} .
$$

Here we will consider the SU(3) structure manifolds, which admit a single globally defined spinor $\eta_{+}$of unitary norm. Hence

$$
\eta_{+}^{1}=|a| e^{i \alpha} \eta_{+}, \eta_{+}^{2}=|b| e^{i \beta} \eta_{+},
$$

where $|a|$ and $|b|$ are clearly the norms of $\eta^{1,2}$. The corresponding pure spinors $\Psi_{ \pm}$on $E$ are

$$
\begin{aligned}
& \Psi_{+}=e^{-\phi} e^{-B} \frac{8}{\left\|\Phi_{+}\right\|} \Phi_{+}, \\
& \Psi_{-}=e^{-\phi} e^{-B} \frac{8}{\left\|\Phi_{-}\right\|} \Phi_{-},
\end{aligned}
$$

with $\left\|\Phi_{ \pm}\right\|=|a|^{2}=|b|^{2}$. The phases of the two pure spinor are $\theta_{+}=\alpha-\beta$ and $\theta_{-}=\alpha+\beta$. $J$ is the Kähler form and $\Omega$ the holomorphic three-form on $M$.

Note that, while the bispinors are globally defined, the pure spinors $\Psi_{ \pm}$glue nontrivially on the double overlaps:

$$
\Psi_{\alpha}=e^{\mathrm{d} \Lambda_{(\alpha \beta)}} \Psi_{\beta} .
$$

As already mentioned, in the backgrounds we will consider in this paper the generalized tangent bundle is trivial and the dilaton is constant. In these cases, we can identify $\Psi_{ \pm}$and $\Phi_{ \pm}$.

The $\mathrm{O}(d, d)$ action on pure spinors is given by its spinorial representation

$$
O \cdot \Psi=e^{-\frac{1}{4} \Theta_{M N}\left[\Gamma^{M}, \Gamma^{N}\right]} \cdot \Psi,
$$

where $\Gamma^{M}$ are the $\operatorname{Cliff}(d, d)$ gamma matrices $\left(\Gamma^{m}=\mathrm{d} x^{m}\right.$ and $\left.\Gamma_{m}=\iota_{m}\right)$ and $\Theta_{M N}$ are the $\mathrm{O}(d, d)$ parameters

$$
\Theta_{M N}=\left(\begin{array}{cc}
a_{n}^{m} & \beta^{m n} \\
b_{m n} & -a_{m}^{n}
\end{array}\right)
$$

Here $a_{n}^{m}, b_{m n}$ and $\beta^{m n}$ parametrise the GL(d) transformations, $B$-transforms and $\beta$ transform, respectively. Then the twist action (2.22) on the spinor reads [28]

$$
O_{\mathrm{tw}} \cdot \Psi=\frac{1}{\sqrt{\operatorname{det} A}} e^{-t\left[a d_{\partial_{t}}(\mathfrak{n})\right]^{m}{ }_{n} e^{n} \wedge \iota_{m}} \cdot \Psi
$$

where $e^{m}$ is a given basis of one-forms on $M$, and $\iota_{m}$ the associated contraction. 


\subsection{Type IIA supersymmetric solutions from twist transformations}

Type IIA supersymmetric compactifications to four-dimensional Minkowski where the internal manifold is the solvmanifold $\mathfrak{g}_{5.17}^{0,0, \pm 1} \times S^{1}$ were found in [40-42]. As shown in section 2.1, this manifold is related by twist to the more general manifold $\mathfrak{g}_{5.17}^{p,-p, \pm 1} \times S^{1}$. It is then natural to ask what is the effect of twisting the solutions in [41, 42].

We will take as starting point Model 3 of [41]. This is an SU(3) structure solution with smeared D6-branes and O6 planes in the directions (146) and (236). For SU(3) structure, the two pure spinors are

$$
\Phi_{+}=\frac{e^{i \theta_{+}}}{8} e^{-i J} \quad \Phi_{-}=-\frac{i}{8} \Omega .
$$

The phase in $\Phi_{+}$is, in general, determined by the orientifold projection. For O6 planes $\theta_{+}$ is actually free and we set it to zero. We take

$$
\Omega=\sqrt{t_{1} t_{2} t_{3}} \chi^{1} \wedge \chi^{2} \wedge \chi^{3} \quad J=\frac{i}{2} \sum_{k} t_{k} \chi^{k} \wedge \bar{\chi}^{k},
$$

with complex structure ${ }^{9}$

$$
\begin{aligned}
\chi^{1} & =e^{1}+i \lambda \frac{\tau_{3}}{\tau_{4}} e^{2}, \\
\chi^{2} & =\tau_{3} e^{3}+i \tau_{4} e^{4}, \\
\chi^{3} & =e^{5}-i \tau_{6} e^{6} .
\end{aligned}
$$

For simplicity, we introduce $\lambda=\frac{t_{2} \tau_{4}^{2}}{t_{1}}$. $e^{i}$ are globally defined one-forms, obtained as in $(2.20)$

$$
e^{m}=\left(A_{2}\right)_{n}^{m} \mathrm{~d} x^{n},
$$

with $A_{2}$ given by (2.36). With this choice the metric is diagonal

$$
g=\operatorname{diag}\left(t_{1}, \lambda t_{2} \tau_{3}^{2}, t_{2} \tau_{3}^{2}, \lambda t_{1}, t_{3}, t_{3} \tau_{6}^{2}\right) .
$$

Positivity of the volume imposes the following constraints on the complex structure and Kähler moduli

$$
\tau_{6}>0, t_{1}, t_{2}, t_{3}>0 .
$$

Due to the presence of intersecting sources, the warp factor is set to one and the dilaton to a constant. By splitting the pure spinor equations (1.1) into forms of fixed degree, it is easy to verify that supersymmetry implies

$$
\begin{aligned}
\mathrm{d}(\operatorname{Im} \Omega) & =0, \\
\mathrm{~d} J & =0, \\
\mathrm{~d}(\operatorname{Re} \Omega) & =g_{s} * F_{2}, \\
F_{6}=F_{4}=F_{0}=H & =0 .
\end{aligned}
$$

${ }^{9} \Omega$ and $J$ are normalised as

$$
\frac{4}{3} J^{3}=i \Omega \wedge \bar{\Omega}=-8 \operatorname{vol}_{(6)}=-8 \sqrt{|g|} e^{1} \wedge e^{2} \wedge e^{3} \wedge e^{4} \wedge e^{5} \wedge e^{6}
$$

where $\operatorname{vol}_{(6)}$ is the internal volume form. 
The only non-zero RR flux reads

$$
g_{s} F_{2}=\frac{\sqrt{\lambda}\left(q_{1} t_{1}-q_{2} t_{2} \tau_{3}^{2}\right)}{\sqrt{t_{3}}}\left(e^{3} \wedge e^{4}-e^{1} \wedge e^{2}\right),
$$

and it is straightforward to check that its Bianchi identity is satisfied. Let us also recall $[6,41]$ the transformation the forms should satisfy under the O6-plane involution $\sigma$ :

$$
\sigma(J)=-J, \sigma(\Omega)=\bar{\Omega}, \sigma(H)=-H, \sigma(F)=\lambda(F) .
$$

Given the directions of the sources here, these orientifold projection conditions are clearly verified by the solution.

Given the solution above, we want to use the twist action to produce solutions, still with O6-planes and D6-branes, on $\mathfrak{g}_{5.17}^{p,-p, \pm 1} \times S^{1}$. The manifolds $\mathfrak{g}_{5.17}^{p,-p, \pm 1} \times S^{1}$ and $\mathfrak{g}_{5.17}^{0,0, \pm 1} \times S^{1}$ are related by the twist matrix $A_{1}$ in (2.36), whose adjoint matrix is

$$
\left.a_{\partial_{5}}(\mathfrak{n})\right|_{p}=\left(\begin{array}{ll}
a_{12} & \\
& a_{34}
\end{array}\right) \quad a_{12}=a_{34}^{T}=\left(\begin{array}{cc}
0 & p q_{1} \\
p q_{2} & 0
\end{array}\right) .
$$

The sixth direction being a trivial circle, we identify $t=x^{5}$. Then the twist action on pure spinors,

$$
\Phi_{ \pm} \mapsto \Phi_{ \pm}^{\prime}=O_{\mathrm{tw}} \Phi_{ \pm}
$$

can be rewritten as

$$
\begin{aligned}
O_{\mathrm{tw}} & =e^{-p x^{5}\left(q_{2} e^{1} \wedge \iota_{2}+q_{1} e^{2} \wedge \iota_{1}\right)} e^{-p x^{5}\left(q_{1} e^{3} \wedge \iota_{4}+q_{2} e^{4} \wedge \iota_{3}\right)} \\
& =O_{12} O_{34}
\end{aligned}
$$

with

$$
\begin{aligned}
O_{12}= & \mathbb{I}+\left[\cosh \left(p \sqrt{q_{1} q_{2}} x^{5}\right)-1\right]\left(e^{1} \wedge \iota_{1}+e^{2} \wedge \iota_{2}+2 e^{1} \wedge e^{2} \wedge \iota_{1} \wedge \iota_{2}\right) \\
& -\frac{1}{\sqrt{q_{1} q_{2}}} \sinh \left(p \sqrt{q_{1} q_{2}} x^{5}\right)\left(q_{2} e^{1} \wedge \iota_{2}+q_{1} e^{2} \wedge \iota_{1}\right), \\
O_{34}= & \mathbb{I}+\left[\cosh \left(p \sqrt{q_{1} q_{2}} x^{5}\right)-1\right]\left(e^{3} \wedge \iota_{3}+e^{4} \wedge \iota_{4}+2 e^{3} \wedge e^{4} \wedge \iota_{3} \wedge \iota_{4}\right) \\
& -\frac{1}{\sqrt{q_{1} q_{2}}} \sinh \left(p \sqrt{q_{1} q_{2}} x^{5}\right)\left(q_{1} e^{3} \wedge \iota_{4}+q_{2} e^{4} \wedge \iota_{3}\right) .
\end{aligned}
$$

Note that unimodularity of the algebra implies $\operatorname{det}(A)=1$. In comparison to the procedure described in [28], here we do not introduce a phase in the twist operator, since we do not modify the nature of the fluxes and sources.

It is straightforward to check that the transformed pure spinors have formally the same expression as in (2.54)-(2.57) but with the one-forms $e^{i}$ now given by

$$
e^{m}=\left(A_{1} A_{2}\right)_{n}^{m} \mathrm{~d} x^{n} .
$$

Also the metric, which is completely specified by the pure spinors, has the same form as for the initial solution, but in the new $e^{i}$ basis

$$
g=\operatorname{diag}\left(t_{1}, \lambda t_{2} \tau_{3}^{2}, t_{2} \tau_{3}^{2}, \lambda t_{1}, t_{3}, t_{3} \tau_{6}^{2}\right) .
$$


In order for the twist transformation to produce new solutions, the transformed pure spinors should again satisfy the supersymmetry equations

$$
\begin{aligned}
\mathrm{d}_{H^{\prime}}\left(\Phi_{+}^{\prime}\right) & =0, \\
\mathrm{~d}_{H^{\prime}}\left(\operatorname{Re} \Phi_{-}^{\prime}\right) & =0, \\
\mathrm{~d}_{H^{\prime}}\left(\operatorname{Im} \Phi_{-}^{\prime}\right) & =g_{s} R^{\prime},
\end{aligned}
$$

where $R^{\prime}$ is the new $\mathrm{RR}$ field $R=\frac{1}{8} * \lambda(F)$. The conditions

$$
H^{\prime}=0 \quad \mathrm{~d} J^{\prime}=0
$$

are automatically satisfied, so that the first two equations in (2.74) reduce to ${ }^{10}$

$$
0=\mathrm{d}\left(\operatorname{Im} \Omega^{\prime}\right)=-p(\lambda-1) \tau_{3} \tau_{6} \sqrt{t_{1} t_{2} t_{3}}\left(q_{2} e^{1} \wedge e^{4} \wedge e^{5}+q_{1} e^{2} \wedge e^{3} \wedge e^{5}\right) \wedge e^{6} .
$$

From this we see that, in addition to $p=0$ case, supersymmetric solutions exist for $p \neq 0$ provided $\lambda=1$.

The last equation in (2.74) defines the transformed RR field

$$
g_{s} R^{\prime}=g_{s} O_{\mathrm{tw}} \cdot R+\mathrm{d}_{H^{\prime}}\left(O_{\mathrm{tw}}\right) \cdot \operatorname{Im} \Phi_{-} .
$$

Since the twist operator does not change the degree of forms, it follows from (2.78) that no new RR fluxes have been generated

$$
F_{0}=F_{4}=F_{6}=0
$$

and (we have already set $\lambda=1$ )

$$
g_{s} F_{2}=\frac{q_{1} t_{1}-q_{2} t_{2} \tau_{3}^{2}}{\sqrt{t_{3}}}\left(e^{3} \wedge e^{4}-e^{1} \wedge e^{2}\right)+\frac{p\left(q_{1} t_{1}+q_{2} t_{2} \tau_{3}^{2}\right)}{\sqrt{t_{3}}}\left(e^{2} \wedge e^{4}+e^{1} \wedge e^{3}\right) .
$$

The Bianchi identity for $F_{2}$ is satisfied

$$
g_{s} \mathrm{~d} F_{2}=c_{1} v^{1}+c_{2} v^{2},
$$

with $v^{1}=t_{1} \sqrt{t_{3}} e^{1} \wedge e^{4} \wedge e^{5}$ and $v^{2}=t_{2} \tau_{3}^{2} \sqrt{t_{3}} e^{2} \wedge e^{3} \wedge e^{5}$ being the covolumes of the sources in (236) and (146). Let us note that the orientifold projection conditions (2.66) are again satisfied with such sources. The sign of the charges

$$
\begin{aligned}
& c_{1}=\frac{2 q_{2}}{t_{3} t_{1}}\left[t_{1} q_{1}\left(1-p^{2}\right)-\left(1+p^{2}\right) t_{2} q_{2} \tau_{3}^{2}\right] \\
& c_{2}=\frac{2 q_{1}}{t_{3} t_{2} \tau_{3}^{2}}\left[\tau_{3}^{2} t_{2} q_{2}\left(1-p^{2}\right)-\left(1+p^{2}\right) t_{1} q_{1}\right]
\end{aligned}
$$

\footnotetext{
${ }^{10}$ Note that a slightly more general solution given by $\chi^{1}=e^{1}+i\left(\frac{\tau_{3}}{\tau_{4}} \lambda e^{2}-\frac{\tau_{2}}{\tau_{4}} e^{3}\right), \chi^{2}=\tau_{2} e^{2}+\tau_{3} e^{3}+$ $i \tau_{4} e^{4}$ and the same $\chi^{3}$ leads to the same $\mathrm{d}\left(\operatorname{Im} \Omega^{\prime}\right)$ and to

$$
\mathrm{d}\left(J^{\prime}\right)=-p(\lambda-1) \tau_{2} \sqrt{\frac{t_{1} t_{2}}{\lambda}}\left(q_{2} e^{1} \wedge e^{4} \wedge e^{5}+q_{1} e^{2} \wedge e^{3} \wedge e^{5}\right)
$$

A supersymmetric solution, requiring $\mathrm{d}(\operatorname{Im} \Omega)=\mathrm{d} J=0$, needs $\lambda=1$. For $\tau_{2}=0$ we can have nonsupersymmetric configurations with a closed $J^{\prime}$.
} 
depends on the parameters, but the sum of the two charges is clearly negative. This guarantees that the transformed background with $p \neq 0$ and $\lambda=1$ is indeed a solution of the full set of ten-dimensional equations of motion. In the next section we will use the nonsupersymmetric version, with $\lambda \neq 1$, as starting point for our search for de Sitter solution.

In the literature, de Sitter backgrounds are often given in terms of SU(3) structure torsions,

$$
\begin{aligned}
\mathrm{d} J & =\frac{3}{2} \operatorname{Im}\left(\bar{W}_{1} \Omega\right)+W_{4} \wedge J+W_{3} \\
\mathrm{~d} \Omega & =W_{1} J^{2}+W_{2} \wedge J+\bar{W}_{5} \wedge \Omega,
\end{aligned}
$$

where $W_{1}$ is a complex scalar, $W_{2}$ is a complex primitive $(1,1)$ form, $W_{3}$ is a real primitive $(2,1)+(1,2)$ form, $W_{4}$ is a real vector and $W_{5}$ is a complex $(1,0)$ form. For the more general $\mathrm{SU}(3)$ structure solution $\left(p \neq 0, \lambda \neq 1, \tau_{2} \neq 0\right)$ mentioned in footnote 10 , we obtain

$$
\begin{aligned}
& W_{1}=\frac{p \tau_{2}(A+B)(1-\lambda)}{6\left(\tau_{2}^{2}+\lambda \tau_{3}^{2}\right) \sqrt{t_{1} t_{2} t_{3}}} \\
& W_{2}=\frac{1}{6\left(\tau_{2}^{2}+\lambda \tau_{3}^{2}\right) \sqrt{t_{1} t_{2} t_{3}}}\left[-i t_{1}\left(p \tau_{2}(A+B)(\lambda+2)+3 \lambda \tau_{3}(A-B)\right) \chi^{1} \wedge \bar{\chi}^{1}+\right. \\
& +3 \sqrt{\lambda t_{1} t_{2}}\left(\tau_{2}(B-A)+p \tau_{3}(\lambda A+B)\right) \chi^{1} \wedge \bar{\chi}^{2}-3 \sqrt{\lambda t_{1} t_{2}}\left(\tau_{2}(B-A)+p \tau_{3}(A+\lambda B)\right) \chi^{2} \wedge \bar{\chi}^{1}+ \\
& \left.+i t_{2}\left(p \tau_{2}(A+B)(1+2 \lambda)+3 \lambda \tau_{3}(A-B)\right) \chi^{2} \wedge \bar{\chi}^{2}-i p \tau_{2} t_{3}(A+B)(\lambda-1) \chi^{3} \wedge \bar{\chi}^{3}\right] \\
& W_{3}=\frac{i p \tau_{2}(\lambda-1)}{8\left(\tau_{2}^{2}+\lambda \tau_{3}^{2}\right)}\left[(A+B) \chi^{1} \wedge \chi^{2} \wedge \bar{\chi}^{3}-(A+B) \chi^{3} \wedge \bar{\chi}^{1} \wedge \bar{\chi}^{2}+\right. \\
& \left.-(A-B)\left(\chi^{1} \wedge \chi^{3} \wedge \bar{\chi}^{2}-\chi^{1} \wedge \bar{\chi}^{2} \wedge \bar{\chi}^{3}+\chi^{2} \wedge \chi^{3} \wedge \bar{\chi}^{1}-\chi^{2} \wedge \bar{\chi}^{1} \wedge \bar{\chi}^{3}\right)\right] \\
& W_{4}=0 \\
& W_{5}=\frac{i p \sqrt{\lambda} \tau_{3}(A+B)(\lambda-1)}{4\left(\tau_{2}^{2}+\lambda \tau_{3}^{2}\right) \sqrt{t_{1} t_{2}}} \chi^{3} \\
& \text { with } A=q_{1} t_{1}, B=q_{2} t_{2}\left(\tau_{3}^{2}+\frac{\tau_{2}^{2}}{\lambda}\right) \text {. }
\end{aligned}
$$

\subsubsection{Localizing the sources and warping}

The supersymmetric solution discussed in the previous section is global, the warp factor and the dilaton being constant. It is an interesting question to see whether localised solutions also exist (see e.g. [27] for a recent discussion about the importance of warping). The strategy for finding localized solutions used in [41] was first to look for a smeared solution at large volume and then localize it by scaling the vielbeine, longitudinal and transverse with respect to the source, with $e^{A}$ and $e^{-A}$, respectively. This procedure works in a number of cases, provided only parallel sources are present. Unfortunately this is not the case for the supersymmetric solution we took as a departure point for our construction the intersecting O6/D6 solution on $s$ 2.5.

It is however possible to find a completely localised solution on $s 2.5$ with O6 planes. The solution has a simpler form in a basis where the algebra is $(25,-15, r 45,-r 35,0,0)$, $r^{2}=1$. In this basis the O6-plane is along the directions (345). 
The $\mathrm{SU}(3)$ structure is constructed as in (2.55) with

$$
\begin{aligned}
\chi^{1} & =e^{-A} e^{1}+i e^{A}\left(\tau_{3} e^{3}+\tau_{4} e^{4}\right), \\
\chi^{2} & =e^{-A} e^{2}+i e^{A} r\left(-\tau_{4} e^{3}+\tau_{3} e^{4}\right), \\
\chi^{3} & =e^{A} e^{5}+i e^{-A} r \tau_{6} e^{6} \\
\tau_{6} & >0, t_{1}=t_{2}, t_{3}>0
\end{aligned}
$$

where the non-trivial warp factor, $A$, depends on $x^{1}, x^{2}, x^{6}$. The metric is diagonal

$$
g=\operatorname{diag}\left(t_{1} e^{-2 A}, t_{1} e^{-2 A}, t_{1}\left(\tau_{3}^{2}+\tau_{4}^{2}\right) e^{2 A}, t_{1}\left(\tau_{3}^{2}+\tau_{4}^{2}\right) e^{2 A}, t_{3} e^{2 A}, t_{3} \tau_{6}^{2} e^{-2 A}\right),
$$

and the only non-zero flux is the RR two-form

$g_{s} F_{2}=-r\left[\tau_{6} \sqrt{t_{3}} \partial_{1}\left(e^{-4 A}\right) \mathrm{d} x^{2} \wedge e^{6}-\tau_{6} \sqrt{t_{3}} \partial_{2}\left(e^{-4 A}\right) \mathrm{d} x^{1} \wedge e^{6}+\frac{1}{\tau_{6}} \sqrt{\frac{t_{1}^{2}}{t_{3}}} \partial_{6}\left(e^{-4 A}\right) \mathrm{d} x^{1} \wedge \mathrm{d} x^{2}\right]$.

Setting the parameters $t_{1}=t_{2}$ in the Kähler form (2.55) allows to have a single source term in the $F_{2}$ Bianchi identity

$$
g_{s} \mathrm{~d} F_{2} \sim e^{-A} \Delta\left(e^{-4 A}\right) e^{1} \wedge e^{2} \wedge e^{6},
$$

where $\Delta$ is the laplacian with unwarped metric.

As $A \rightarrow 0$ this solution becomes fluxless ( $s 2.5$ can indeed support such solutions), hence it cannot be found following the strategy of localizing the large volume smeared solutions. Unfortunately this solution does not satisfy the twist to $p \neq 0,(2.30)$, since for $p \neq 0$ the action of the involution of an O6-plane with a component along direction 5 is not compatible with the algebra.

\subsection{A digression: twist and non-geometric backgrounds}

We would like to come back to the question of the consistency of the twist transformation. As explained already, the transformation is obstructed unless the matrix $A$ is conjugated to an integer-valued matrix. In many cases, the twist can result in a topology change similar to what is achieved by T-duality. The latter also can be obstructed, and yet these obstructions do not stop us from performing the duality transformation. So what about the obstructed twist?

To keep things simple, let us consider again an almost abelian algebra and the gluing under $t \rightarrow t+t_{0}$. We should have in general

$$
T_{6}:\left\{\begin{array}{l}
t \rightarrow t+t_{0} \\
x^{i} \rightarrow \tilde{A}_{M}\left(-t_{0}\right)_{j}^{i} x^{j}
\end{array} \quad i, j=1, \ldots, 5,\right.
$$

where $\tilde{A}_{M}\left(-t_{0}\right)$ is necessarily an integer-valued matrix for $t_{0} \neq 0$. In the case of compact solvmanifolds this matrix is given by (2.22). For the algebras that do not admit an action of a lattice, $\tilde{A}_{M}\left(-t_{0}\right)$ has nothing to do with the algebra. Then the one forms $e^{i}=A(t)^{i}{ }_{j} \mathrm{~d} x^{j}$ $\left(\mathrm{d} x^{6}=\mathrm{d} t\right)$ are defined only locally and have discontinuities under $t \rightarrow t+t_{0}$. These kinds 
of discontinuity are actually familiar from the situations when an obstructed T-duality is performed, and are commonly referred to as non-geometric backgrounds. One way to see this is to work on the generalized tangent bundle and use local $O(6) \times O(6)$ transformations (for six-dimensional internal manifolds) to bring the generalized vielbeine to the canonical lower diagonal form (2.46). In geometric backgrounds, this is a good transformation, while in the non-geometric case it involves non-single valued functions [43].

As an example, let us consider the manifold $\mathfrak{g}_{4.2}^{-p} \times T^{2}$, where the algebra $\mathfrak{g}_{4.2}^{-p}$ is given in appendix A.2. The corresponding group does not admit a lattice. For generic $p$ this is very easy to see since the group is not unimodular. For $p=2$, the group is unimodular but there still is no lattice. As explained in the appendix A.2, in this case, the characteristic polynomial cannot have integer coefficients, and therefore there is an obstruction to the existence of a lattice.

If we now consider the algebra together with its dual, i.e. examine the existence of a lattice on the generalized tangent bundle, we should study the $6 \times 6$ matrix $M(t)=$ $\operatorname{diag}\left(\mu(t), \mu(-t)^{T}\right)$ instead of the matrix $\mu(t)$. One has

$$
M(t)=\left(\begin{array}{cccccc}
e^{p t} & 0 & 0 & 0 & 0 & 0 \\
0 & e^{-t} & 0 & 0 & 0 & 0 \\
0 & -t e^{-t} & e^{-t} & 0 & 0 & 0 \\
0 & 0 & 0 & e^{-p t} & 0 & 0 \\
0 & 0 & 0 & 0 & e^{t} & t e^{t} \\
0 & 0 & 0 & 0 & 0 & e^{t}
\end{array}\right)
$$

For $t_{0}=\ln \left(\frac{3+\sqrt{5}}{2}\right)$ and $p \in \mathbb{N}^{*}, M\left(t=t_{0}\right)$ is conjugated to an integer matrix, $P^{-1} M\left(t_{0}\right) P=$ $N$, where $N$ is an integer matrix (Theorem 8.3.2 in [33]):

$$
\begin{aligned}
P & =\left(\begin{array}{cccccc}
1 & 0 & 0 & \frac{18+8 \sqrt{5}}{7+3 \sqrt{5}} & 0 & 0 \\
0 & 1 & 0 & 0 & 0 & \frac{2(2+\sqrt{5})}{3+\sqrt{5}} \\
0 & 0 & \ln \left(\frac{2}{3+\sqrt{5}}\right) & 0 & \frac{2(2+\sqrt{5}) \ln \left(\frac{3+\sqrt{5}}{2}\right)}{3+\sqrt{5}} & 0 \\
1 & 0 & 0 & \frac{2}{3+\sqrt{5}} & 0 & 0 \\
0 & 0 & \ln \left(\frac{2}{3+\sqrt{5}}\right) & 0 & -\frac{(1+\sqrt{5}) \ln \left(\frac{3+\sqrt{5}}{2}\right)}{3+\sqrt{5}} & 0 \\
0 & -1 & 0 & 0 & 0 & \frac{1+\sqrt{5}}{3+\sqrt{5}}
\end{array}\right) \\
N & =\left(\begin{array}{cccccc}
a_{11} & 0 & 0 & a_{14} & 0 & 0 \\
0 & 2 & 0 & 0 & 0 & -1 \\
0 & 2 & 2 & 0 & 1 & -1 \\
a_{41} & 0 & 0 & a_{44} & 0 & 0 \\
0 & 1 & 1 & 0 & 1 & -1 \\
0 & -1 & 0 & 0 & 0 & 1
\end{array}\right)
\end{aligned}
$$

The piece

$$
N_{4}=\left(\begin{array}{ll}
a_{11} & a_{14} \\
a_{41} & a_{44}
\end{array}\right)=\left(\begin{array}{cc}
0 & -1 \\
1 & 3
\end{array}\right)^{p}
$$


comes from the entries $e^{p t}$ and the result can be obtained ${ }^{11}$ from (A.14). We see that on the generalized tangent bundle the basic obstruction to the existence of a lattice is easily removed. Moreover it is not hard to see that, due to putting together the algebra and its dual, even the requirement of unimodularity can be dropped.

On the generalized tangent bundle we can therefore obtain a lattice. For non-geometry, one may ask for more: the integer matrix $N$ being in $O(3,3)$. This question can be decomposed into $N_{4} \in O(1,1)$ and the $4 \times 4$ integer matrix in $O(2,2)$. Actually, the latter is true. ${ }^{12}$ But $N_{4} \notin O(1,1)$. Moreover, one can prove that $\operatorname{diag}\left(e^{p t}, e^{-p t}\right)$ can only be conjugated to an integer $O(1,1)$ matrix for $t=0$. Indeed, the eigenvalues of an integer $O(1,1)$ matrices are \pm 1 , and those are not changed by conjugation.

This is reminiscent of the twist construction of the IIB background $n 3.14$ discussed in [28]. The internal manifold is a circle fibration over a five manifold $M_{5}$, which itself is a bundle with a two-torus fiber, but the only obvious duality seen there is the $O(2,2)$ associated with the two-torus. The solution on $M_{5} \times S^{1}$ is obtained from IIB solution on $\mathbb{T}^{6}$ with a self-dual three-form flux, but not $n 3.14$ itself [41].

By taking $p=0$ in (2.90), we obtain a different topology. In $M(t)$ the corresponding direction becomes trivial, and we can forget about it. Up to an $O(1,1)$ action, the nontrivial part of $M(t)$ can still be thought of as corresponding to the algebra on $T\left(\varepsilon_{1,1}\right) \oplus$ $T^{*}\left(\varepsilon_{1,1}\right)$. Indeed, $\varepsilon_{1,1}$ has two local isometries, and T-duality (the $O(1,1)$ in question) with respect to any of them will yield a non-geometric background. This can be inferred by simply noticing that the result of the duality in (any direction) is not unimodular; more detailed discussion of T-duality on $\varepsilon_{1,1}$ can be found in appendix B.

A better understanding of the orientifold planes in generalized complex geometry is needed in order to apply the twist transformation to constructing non-geometric backgrounds. However, the possibility of using solvable algebras in order to describe (some of) these is interesting.

\section{Supersymmetry breaking and de Sitter vacua}

In the literature on de Sitter backgrounds, O6/D6 models seem to have good chances at yielding a solution which can be embedded in string theory, at least in the conservative approach of "geometric" compactifications. We shall concentrate on the resolution of the ten-dimensional equations of motion in this conservative set-up, making use of the technology described in the last section and adapting it to the description of non-supersymmetric configurations.

We consider type IIA supergravity and mostly follow the conventions of [44, 45]; we differ in the definition of the Hodge star where we have an extra sign depending on the

\footnotetext{
${ }^{11}$ Another possible conjugation is given in (2.18). The other part of $N$, the $4 \times 4$ integer matrix, can also be different, see the change of basis in Proposition 7.2.9 in [33].

${ }^{12}$ Note it is not true for the one given in Proposition 7.2.9 of [33].
} 
parity of the forms. ${ }^{13}$ In particular,

$$
F_{p} \wedge \hat{*} F_{p}=\mathrm{d}^{10} x \sqrt{\left|g_{10}\right|}(-1)^{(10-p) p} \frac{F_{\mu_{1} \ldots \mu_{p}} F^{\mu_{1} \ldots \mu_{p}}}{p !}=\mathrm{d}^{10} x \sqrt{\left|g_{10}\right|}(-1)^{(10-p) p}\left|F_{p}\right|^{2} .
$$

We explicitly denote the ten-dimensional Hodge star by $\hat{*}$, reserving the symbol $*$ for its six-dimensional counterpart.

In order to derive the ten-dimensional equations of motion, we shall need source terms, and to this end let us consider the DBI action of only one $\mathrm{D} p$-brane in string frame

$$
S_{s}=-T_{p} \int \mathrm{d}^{p+1} x e^{-\phi} \sqrt{\left|i^{*}\left[g_{10}\right]+\mathcal{F}\right|}, \quad T_{p}^{2}=\frac{\pi}{\kappa^{2}}\left(4 \pi^{2} \alpha^{\prime}\right)^{3-p} .
$$

Here $T_{p}$ is the tension of the brane; for an O-plane, one has to replace $T_{p}$ by $-2^{p-5} T_{p}$. The open string excitations will not be important for our solution, and we shall discard the $\mathcal{F}$ contribution from now on (note as well that the $B$-field will pull back to zero along the sources).

To derive the equations of motion, a priori, we should take a full variation of the DBI action with respect to the bulk metric. For supersymmetry preserving (calibrated) sources, there exists a convenient way of dealing with this. In this case, one can think of an expansion of the DBI action around the supersymmetric configuration and, to leading order, replace the DBI action by a pullback of the calibration form. As discussed around (1.16), it is given in terms of the non-closed pure spinor discussed in the previous section: $\Phi_{-}$ in type IIA. As shown in [6], this allows to prove that, for Minkowski compactifications, the equations of motion follow from the first order pure spinor equations, and the flux Bianchi identities. A similar treatment of space-time filling sources is also possible for nonsupersymmetric Minkowski and $A d S_{4}$ configurations [11]. It is worth stressing that, even in these cases, the sources continue being (generalized) calibrated and are not responsible for the supersymmetry breaking. However convenient, as we shall see, these kinds of source are not going to be helpful in our search for a dS vacuum.

At this point we shall consider an important assumption: inspired by the supersymmetric case just described, we make a proposal for sources breaking the bulk supersymmetry. The latter can be applied in the case of an internal space with $\mathrm{SU}(3)$ structure, and the triviality of the canonical bundle is going to be important. We shall assume that, in analogy with the supersymmetric case, the DBI action can be replaced to leading order by the pullback of a (poly)form $X$ in the bulk, as discussed around (1.19). The bulk does

\footnotetext{
${ }^{13}$ In IIA, the sign is always positive on RR fields, but not on the odd forms, $H$ and $\mathrm{d} \phi$, hence the sign difference with respect to [45] for the corresponding terms in the action. The sign difference is related to the fact we use the Mukai pairing to give the norm (see footnote 1): for a real form $\alpha_{i}$, we have $\left\langle * \lambda\left(\alpha_{i}\right), \alpha_{i}\right\rangle=\left|\alpha_{i}\right|^{2} \times$ vol. Note that these conventions are consistent with the SUSY conditions written before. There is a factor of 2 difference in the normalization of the RR kinetic terms with respect to [15], which will result in a difference in the RR quantization conditions. For a $k$-flux $\alpha$ through a $k$-cycle $\Sigma$ (with embedding $i$ into the bulk manifold $M$ ), we have
}

$$
\frac{1}{\left(2 \pi \sqrt{\alpha^{\prime}}\right)^{k-1}} \frac{1}{\operatorname{vol}_{M}} \int_{\Sigma} i^{*} \alpha=\frac{1}{\left(2 \pi \sqrt{\alpha^{\prime}}\right)^{k-1}} \frac{1}{\operatorname{vol}_{M}} \int_{M}\langle\delta(\Sigma \hookrightarrow M), \alpha\rangle=n,
$$

where $n$ is an integer. 
have invariant forms and hence pure spinors can be constructed, but $X$ cannot be pure, otherwise the source would preserve bulk supersymmetry. The form $X$ is expandable in the Hodge diamond defined by the pure spinors. This amounts to consider forms that are equivalent not to simply the invariant spinor $\eta_{+}$(defining the $\mathrm{SU}(3)$ structure) but to a full spinorial basis, $\eta_{+}, \eta_{-}, \gamma^{\bar{i}} \eta_{+}$and $\gamma^{i} \eta_{-}$, where $i, \bar{i}=1, \ldots 3$ are the internal holomorphic and antiholomorphic indices. ${ }^{14}$ To be concrete we shall consider a generic odd form

$$
\begin{aligned}
X=\sqrt{\left|g_{4}\right|} \mathrm{d}^{4} x \wedge X_{-}= & \sqrt{\left|g_{4}\right|} \mathrm{d}^{4} x \wedge\left(\operatorname{Re} X_{-}+i \operatorname{Im} X_{-}\right) \\
X_{-}=\operatorname{Re} X_{-}+i \operatorname{Im} X_{-}= & \frac{8}{\| \Phi_{-}||}\left(\alpha_{0} \Phi_{-}+\widetilde{\alpha}_{0} \bar{\Phi}_{-}+\alpha_{m n} \gamma^{m} \Phi_{-} \gamma^{n}+\widetilde{\alpha}_{m n} \gamma^{m} \bar{\Phi}_{-} \gamma^{n}\right. \\
& \left.+\alpha_{m}^{L} \gamma^{m} \Phi_{+}+\widetilde{\alpha}_{m}^{L} \gamma^{m} \bar{\Phi}_{+}+\alpha_{n}^{R} \Phi_{+} \gamma^{n}+\widetilde{\alpha}_{n}^{R} \bar{\Phi}_{+} \gamma^{n}\right),
\end{aligned}
$$

where $\Phi_{ \pm}$are given in (2.54) and the $\gamma^{\prime}$ s act on even and odd forms via contractions and wedges

$$
\gamma^{m} \Phi_{ \pm}=\left(g^{m n} \imath_{n}+\mathrm{d} x^{m}\right) \Phi_{ \pm}, \quad \text { and } \quad \Phi_{ \pm} \gamma^{m}=\mp\left(g^{m n} \imath_{n}-\mathrm{d} x^{m}\right) \Phi_{ \pm} .
$$

The action for a single source term becomes

$$
\begin{aligned}
S_{s} & =-T_{p} \int_{\Sigma} \mathrm{d}^{p+1} x e^{-\phi} \sqrt{\left|i^{*}\left[g_{10}\right]\right|} \\
& =-T_{p} \int_{\Sigma} e^{-\phi} i^{*}[\operatorname{Im} X] \\
& =-T_{p} \int_{M_{10}} e^{-\phi}\left\langle j_{p}, \operatorname{Im} X\right\rangle \\
& =T_{p} \int_{M_{10}} \mathrm{~d}^{10} x \sqrt{\left|g_{10}\right|} e^{-\phi_{\hat{*}}\left\langle j_{p}, \operatorname{Im} X\right\rangle,}
\end{aligned}
$$

where $i: \Sigma \hookrightarrow M_{10}$ is the embedding of the subspace $\Sigma$ wrapped by the source in the bulk and $j_{p}=\delta\left(\Sigma \hookrightarrow M_{10}\right)$ is the dimensionless Poincaré dual of $\Sigma$. The change of sign between the last two lines is due to the Lorentzian signature which gives a minus when taking the Hodge star. For the sum of all sources we then take the action

$$
\left.S_{s}=T_{p} \int_{M_{10}} \mathrm{~d}^{10} x \sqrt{\left|g_{10}\right|} e^{-\phi_{\hat{*}}\langle j}, \operatorname{Im} X\right\rangle, \quad j=\sum_{D p} j_{p}-\sum_{O p} 2^{p-5} j_{p} .
$$

As discussed after (1.19), this replacement of the source action is for now only a proposal and we hope to provide a justification for it in future work. Our interpretation is that sources remain standard D-branes or O-planes, but their embedding into $M$, in particular the form which describes the subspace wrapped by them, is modified from $\operatorname{Im} \Phi_{-}$to the

\footnotetext{
${ }^{14}$ The covariant derivative on the invariant spinor contains the same information as the intrinsic torsions. For the explicit dictionary for $\mathrm{SU}(3)$ structure see [46]. In the supersymmetric backgrounds the ( $H$-twisted) derivative on the spinor cancels against the RR contribution [9], and the entire content of that cancellation is captured by first order equation on the pure spinors (1.1). For the non-supersymmetric backgrounds, the unbalance between the NS and RR contributions results in the presence of terms that need to be expanded in the full basis (see e.g. [11]).
} 
more general $\operatorname{Im} X_{-}$. As mentioned in the Introduction, a difference with the supersymmetric case is that we are not sure anymore that the equations of motion derived from both actions are the same. Our procedure will consist in finding solutions to the equations derived from the proposed source action, which are much easier to deal with. We will then argue that these solutions are also solutions of the equations derived from the standard source action. Until this is done in section 3.3, we mean by solution a solution to the equations of motion derived with our proposed source action.

In the following, we will consider solutions where the only non-trivial fluxes are $H, F_{0}$ and $F_{2}$ on the internal manifold, and the RR magnetic sources are $D 6$ 's and $O 6$ 's. The sources will be smeared, so we take $\delta \rightarrow 1$ and the warp factor $e^{2 A}=1$. The relevant part of the action, ${ }^{15}$ in string frame, is then

$$
S=\frac{1}{2 \kappa^{2}} \int \mathrm{d}^{10} x \sqrt{\left|g_{10}\right|}\left[e^{-2 \phi}\left(R_{10}+4|\nabla \phi|^{2}-\frac{1}{2}|H|^{2}\right)-\frac{1}{2}\left(\left|F_{0}\right|^{2}+\left|F_{2}\right|^{2}\right)+2 \kappa^{2} T_{p} e^{\left.-\phi_{\hat{*}}\langle j, \operatorname{Im} X\rangle\right],}\right.
$$

where $2 \kappa^{2}=(2 \pi)^{7}\left(\alpha^{\prime}\right)^{4}$.

With the flux ansatz (1.6), the flux equations of motion and Bianchi identities reduce to the six-dimensional equations

$$
\begin{aligned}
\mathrm{d} H & =0, \\
\mathrm{~d} F_{0} & =0, \\
\mathrm{~d} F_{2}-H \wedge F_{0} & =2 \kappa^{2} T_{p} j, \\
H \wedge F_{2} & =0, \\
\mathrm{~d}\left(e^{-2 \phi} * H\right) & =-F_{0} \wedge * F_{2}-e^{-\phi} 4 \kappa^{2} T_{p} j \wedge \operatorname{Im} X_{1}, \\
\mathrm{~d}\left(* F_{2}\right) & =0,
\end{aligned}
$$

where $\operatorname{Im} X_{1}$ is the one-form part of $\operatorname{Im} X_{-}$in (3.3). ${ }^{16}$

The ten-dimensional Einstein and dilaton equations in string frame now become

$$
\begin{gathered}
R_{M N}-\frac{g_{M N}}{2} R_{10}= \\
2 g_{M N}\left(\nabla^{2} \phi-2|\nabla \phi|^{2}\right)-2 \nabla_{M} \nabla_{N} \phi+\frac{1}{4} H_{M P Q} H_{N}{ }^{P Q}+\frac{e^{2 \phi}}{2} F_{2}{ }_{M P} F_{2}{ }^{P} \\
-\frac{g_{M N}}{2}\left(-4|\nabla \phi|^{2}+\frac{1}{2}|H|^{2}+\frac{e^{2 \phi}}{2}\left(\left|F_{0}\right|^{2}+\left|F_{2}\right|^{2}\right)\right)+e^{\phi} \frac{1}{2} T_{M N}, \\
8\left(\nabla^{2} \phi-|\nabla \phi|^{2}\right)+2 R_{10}-|H|^{2}=-e^{\phi} \frac{T_{0}}{p+1} .
\end{gathered}
$$

\footnotetext{
${ }^{15}$ By relevant we mean the parts of the bulk and source actions that give non-trivial contributions to the Einstein and dilaton equations of motion and to the derivation of the four-dimensional effective potential of section 3.4. We do not write down the Chern-Simons terms of the bulk action and the Wess-Zumino part of the source action. Indeed they do not have any metric nor dilaton dependence and, since we do not allow for non-zero values of $\mathrm{RR}$ gauge potentials in the background, they will not contribute to the vacuum value of the four-dimensional potential either. However, both terms contribute the flux e.o.m. and Bianchi identities (in particular, see [45, 47, 48] for a discussion of the Chern-Simons terms in the presence of non-trivial background fluxes).

${ }^{16}$ We refer to [6] for a discussion of the last term in the $H$ equation of motion.
} 
Here $T_{M N}$ and $T_{0}$ are the source energy momentum tensor and its partial trace, respectively $^{17}$

$$
\begin{aligned}
T_{M N} & =2 \kappa^{2} T_{p} \hat{*}\left\langle j, g_{P(M} d x^{P} \otimes \iota_{N)} \operatorname{Im} X-\delta_{(M}^{m} g_{N) n} C_{m}^{n}\right\rangle, \\
T_{0} & =2 \kappa^{2} T_{p} \hat{*}\left\langle j, d x^{N} \otimes \iota_{N} \operatorname{Im} X\right\rangle=(p+1) 2 \kappa^{2} T_{p} \hat{*}\langle j, \operatorname{Im} X\rangle, \\
T & =g^{M N} T_{M N}=T_{0}-2 \kappa^{2} T_{p} \hat{*}\left\langle j, C_{m}^{m}\right\rangle .
\end{aligned}
$$

$m, n$ are real internal indices, $C_{m}^{n}=\sqrt{\left|g_{4}\right|} \mathrm{d}^{4} x \wedge c_{m}^{n}$ and

$$
\begin{aligned}
c_{m}^{n}= & \frac{8}{\left\|\Phi_{-}\right\|} \operatorname{Im}\left(\alpha_{m}^{L} \gamma^{n} \Phi_{+}+\widetilde{\alpha}_{m}^{L} \gamma^{n} \bar{\Phi}_{+}+\alpha_{m}^{R} \Phi_{+} \gamma^{n}+\widetilde{\alpha}_{m}^{R} \bar{\Phi}_{+} \gamma^{n}\right. \\
& \left.+\alpha_{p m} \gamma^{p} \Phi_{-} \gamma^{n}+\alpha_{m p} \gamma^{n} \Phi_{-} \gamma^{p}+\widetilde{\alpha}_{p m} \gamma^{p} \bar{\Phi}_{-} \gamma^{n}+\widetilde{\alpha}_{m p} \gamma^{n} \bar{\Phi}_{-} \gamma^{p}\right) .
\end{aligned}
$$

For supersymmetric configurations, $\operatorname{Im} X_{-}=8 \operatorname{Im} \Phi_{-}, c_{m}^{n}=0, T_{0}$ reduces to the full trace of the source energy-momentum tensor, $T=T_{0}$ and one recovers the formulae in [6].

We can now split (3.8) into its four and six-dimensional components. Since for maximally symmetric spaces, $R_{\mu \nu}=\Lambda g_{\mu \nu}=\left(R_{4} / 4\right) g_{\mu \nu}$, for constant dilaton, $e^{\phi}=g_{s}$, the four-dimensional Einstein equation has only one component and reduces to

$$
R_{4}=-2 R_{6}+|H|^{2}+g_{s}^{2}\left(\left|F_{0}\right|^{2}+\left|F_{2}\right|^{2}\right)-2 g_{s} \tilde{T}_{0}=4 \Lambda
$$

Not to clutter equations, in the rest of the papers we set $\tilde{T}_{0}=T_{0} /(p+1)$.

This equation defines the cosmological constant, $\Lambda$. Using the dilaton equation (3.9), the source contribution can be eliminated and we obtain

$$
\begin{aligned}
R_{4} & =\frac{2}{3}\left[-R_{6}-\frac{g_{s}^{2}}{2}\left|F_{2}\right|^{2}+\frac{1}{2}\left(|H|^{2}-g_{s}^{2}\left|F_{0}\right|^{2}\right)\right], \\
R_{10} & =\frac{1}{3}\left[R_{6}+|H|^{2}-g_{s}^{2}\left(\left|F_{0}\right|^{2}+\left|F_{2}\right|^{2}\right)\right] .
\end{aligned}
$$

We are left with the internal Einstein equation,

$$
R_{m n}-\frac{1}{4} H_{m p q} H_{n}{ }^{p q}-\frac{g_{s}^{2}}{2} F_{2 m p} F_{2 n}{ }^{p}-\frac{g_{m n}}{6}\left[R_{6}-\frac{1}{2}|H|^{2}-\frac{5}{2} g_{s}^{2}\left(\left|F_{0}\right|^{2}+\left|F_{2}\right|^{2}\right)\right]=\frac{g_{s}}{2} T_{m n},
$$

and the dilaton equation

$$
g_{s} \tilde{T}_{0}=\frac{1}{3}\left[-2 R_{6}+|H|^{2}+2 g_{s}^{2}\left(\left|F_{0}\right|^{2}+\left|F_{2}\right|^{2}\right)\right] .
$$

\footnotetext{
${ }^{17}$ In our conventions$$
\frac{1}{\sqrt{\left|g_{10}\right|}} \frac{\delta S_{s}}{\delta \phi}=-\frac{e^{-\phi}}{2 \kappa^{2}} \frac{T_{0}}{p+1}, \quad \frac{1}{\sqrt{\left|g_{10}\right|}} \frac{\delta S_{s}}{\delta g^{M N}}=-\frac{e^{-\phi}}{4 \kappa^{2}} T_{M N} .
$$

To derive (3.11), we considered the fact that each $\gamma_{m}$ matrix in the bispinors $\Phi_{ \pm}$carries one vielbein. To derive $C_{m}^{n}$ the metric dependence of the full Hodge decomposition (3.3) must be taken into account. For supersymmetric cases, the operator $g_{P(M} d x^{P} \otimes \iota_{N)}$ in $T_{M N}$ is the projector on the cycle wrapped by the source [49].
} 
Provided the flux equations of motion and Bianchi identities are satisfied, solving the Einstein and dilaton equations becomes equivalent to finding the correct energy-momentum tensor for the sources. We shall now consider an explicit example and see how the nonsupersymmetric modifications to the energy momentum tensor help in looking for de Sitter solutions. In the process we shall establish some properties of the form $\operatorname{Im} X_{-}$.

\subsection{Solvable de Sitter}

Our starting point is the solution described in section 2.3, based on the algebra

$$
\left(q_{1}(p 25+35), q_{2}(p 15+45), q_{2}(p 45-15), q_{1}(p 35-25), 0,0\right) .
$$

Among the different $\mathrm{O} 6$ projections compatible with the algebra for $p=0$, only those along 146 or 236 are still compatible with the full algebra with $p \neq 0$. In section 2.3 we showed that, acting with a twist transformation on the supersymmetric solution with $p=0$ and the right $\mathrm{O} 6$ planes, one finds a family of backgrounds characterised by the $\mathrm{SU}(3)$ structure

$$
\begin{aligned}
& \Omega=\sqrt{t_{1} t_{2} t_{3}}\left(e^{1}+i \lambda \frac{\tau_{3}}{\tau_{4}} e^{2}\right) \wedge\left(\tau_{3} e^{3}+i \tau_{4} e^{4}\right) \wedge\left(e^{5}-i \tau_{6} e^{6}\right), \\
& J=t_{1} \lambda \frac{\tau_{3}}{\tau_{4}} e^{1} \wedge e^{2}+t_{2} \tau_{3} \tau_{4} e^{3} \wedge e^{4}-t_{3} \tau_{6} e^{5} \wedge e^{6},
\end{aligned}
$$

which satisfy the supersymmetry equations (1.1) only when the parameter $\lambda=\frac{t_{2} \tau_{4}^{2}}{t_{1}}$ is equal to one. One motivation to consider what happens when supersymmetry is violated comes from the form of the Ricci scalar for this class of backgrounds ${ }^{18}$

$$
R_{6}=-\frac{1}{t_{1} t_{2} t_{3} \tau_{3}^{2}}\left[(A-B)^{2}+p^{2}\left(\frac{(\lambda-1)^{2}}{2 \lambda}\left(A^{2}+B^{2}\right)+(A+B)^{2}\right)\right],
$$

where we introduced the following quantities

$$
A=q_{1} t_{1} \quad B=q_{2} t_{2} \tau_{3}^{2} .
$$

\footnotetext{
${ }^{18}$ The Ricci tensor of a group manifold is easily computed in frame indices (where the metric is the unit
} one) in terms of the group structure constants

$$
R_{a d}=\frac{1}{2}\left(\frac{1}{2} f_{a}^{b c} f_{d b c}-f_{d b}^{c} f_{c a}^{b}-f_{a c}^{b} f_{d b}^{c}\right) .
$$

In our case, with the appropriate rescaling of the one-forms $e^{a}$ in (2.72) and of the structure constants, we find that the only non-zero components of the Ricci tensor are

$$
\begin{aligned}
& R_{11}=-R_{22}=\frac{1}{2 t_{1} t_{2} t_{3} \tau_{3}^{2}}\left[A^{2}-B^{2}+\frac{p^{2}}{\lambda}\left(A^{2}-\lambda^{2} B^{2}\right)\right], \\
& R_{33}=-R_{44}=\frac{1}{2 t_{1} t_{2} t_{3} \tau_{3}^{2}}\left[B^{2}-A^{2}+\frac{p^{2}}{\lambda}\left(B^{2}-\lambda^{2} A^{2}\right)\right], \\
& R_{55}=-\frac{1}{t_{1} t_{2} t_{3} \tau_{3}^{2}}\left[(A-B)^{2}+p^{2}\left(\frac{1+\lambda^{2}}{2 \lambda}\left(A^{2}+B^{2}\right)+2 A B\right)\right], \\
& R_{14}=R_{23}=\frac{1}{2 t_{1} t_{2} t_{3} \tau_{3}^{2}} \frac{p}{\sqrt{\lambda}}(\lambda-1)\left(A^{2}-B^{2}\right) .
\end{aligned}
$$

Notice that the curvature only receives contributions from $R_{55}$. 
Indeed, $R_{6}$ gets more negative when the SUSY breaking parameters $p$ and $|\lambda-1|$ leave their SUSY value 0 . Therefore, the value $R_{4}$ as given in (3.16) is lifted by SUSY breaking and this is a priori promising for a de Sitter vacuum.

The rest of this section is devoted to the search of de Sitter solutions on the class of backgrounds discussed above. We will take the same SU(3) structure as in (3.21) and metric

$$
g=\operatorname{diag}\left(t_{1}, \lambda t_{2} \tau_{3}^{2}, t_{2} \tau_{3}^{2}, \lambda t_{1}, t_{3}, t_{3} \tau_{6}^{2}\right)
$$

in the basis of $e^{m}$ given in (2.72). Dilaton and warp factor are still constant: $e^{\phi}=g_{s}$ and $e^{2 A}=1$. For the fluxes, beside the RR two-form, we will allow for non-trivial RR zero-form and NS three-form

$$
\begin{aligned}
H & =h\left(t_{1} \sqrt{t_{3} \lambda} e^{1} \wedge e^{4} \wedge e^{5}+t_{2} \tau_{3}^{2} \sqrt{t_{3} \lambda} e^{2} \wedge e^{3} \wedge e^{5}\right), \\
g_{s} F_{2} & =\gamma \sqrt{\frac{\lambda}{t_{3}}}\left[(A-B)\left(e^{3} \wedge e^{4}-e^{1} \wedge e^{2}\right)+\frac{p}{\lambda}(A+B)\left(\lambda^{2} e^{2} \wedge e^{4}+e^{1} \wedge e^{3}\right)\right], \\
g_{s} F_{0} & =\frac{h}{\gamma} .
\end{aligned}
$$

We have introduced here another parameter $\gamma>0$ which is given by the ratio of NS and RR zero-form fluxes. We consider again D6 or O6 sources along (236) and (146), and one can check that the $S U(3)$ structure forms and the fluxes chosen satisfy the orientifold projection conditions (2.66). Note that the NS flux has component along the covolumes ${ }^{19}$ of the sources, $v^{1}=t_{1} \sqrt{t_{3} \lambda} e^{1} \wedge e^{4} \wedge e^{5}$ and $v^{2}=t_{2} \tau_{3}^{2} \sqrt{t_{3} \lambda} e^{2} \wedge e^{3} \wedge e^{5}$.

The SUSY solutions of section 2.3 are obtained setting

$$
\lambda=1 \text { or } p=0, \gamma=1, F_{0}=h=0 .
$$

\subsubsection{The solution}

We will first consider the four-dimensional Einstein equation (3.16). Using the ansatz for the fluxes we obtain

$$
\begin{aligned}
g_{s}^{2}\left|F_{2}\right|^{2} & =\frac{2 \gamma^{2}}{t_{1} t_{2} t_{3} \tau_{3}^{2}}\left[(A-B)^{2}+p^{2}(A+B)^{2}\left(\frac{(\lambda-1)^{2}}{2 \lambda}+1\right)\right], \\
|H|^{2} & =2 h^{2}
\end{aligned}
$$

Notice that

$$
g_{s}^{2}\left|F_{2}\right|^{2}=2 \gamma^{2}\left[-R_{6}+p^{2} \frac{(\lambda-1)^{2}}{\lambda} \frac{q_{1} q_{2}}{t_{3}}\right] .
$$

This allows to write the four dimensional Ricci scalar as

$$
R_{4}=\frac{2}{3}\left[\left(1-2 \gamma^{2}\right)\left(-R_{6}-\frac{1}{2} g_{s}^{2}\left|F_{0}\right|^{2}\right)+\gamma^{2}\left(-R_{6}-\frac{q_{1} q_{2}}{t_{3}} p^{2} \frac{(\lambda-1)^{2}}{\lambda}\right)\right] .
$$

\footnotetext{
${ }^{19}$ In order not to clutter the notations we did not divide $v^{i}$ by $\sqrt{2}$ (and recalibrate the cycles accordingly) with an unfortunate consequence that $H$ in the normalization discussed in footnote 13 comes out as evenquantized, and $\gamma$ is rational up to multiplication by $\sqrt{2}$.
} 
Since the second bracket is positive (see (3.26)), we see that de Sitter solutions are possible, for instance, for $\gamma^{2} \leq \frac{1}{2}$ and small $F_{0}$. Note also that $R_{4}$ clearly vanishes in the supersymmetric solution where $\lambda=1, \gamma=1$ and $F_{0}=0$.

To solve the dilaton and internal Einstein equations it is more convenient to go to frame indices and take a unit metric. As already discussed in footnote 18, this choice makes the computation of the Ricci tensor very simple. To simplify notations we introduce the constant

$$
C=-\frac{1}{6}\left(R_{6}-\frac{1}{2}|H|^{2}-\frac{5}{2} g_{s}^{2}\left(\left|F_{0}\right|^{2}+\left|F_{2}\right|^{2}\right)\right) .
$$

Then the dilaton equation becomes

$$
g_{s} \tilde{T}_{0}=4 C-\frac{h^{2}}{\gamma^{2}}-\frac{2 \gamma^{2}}{t_{1} t_{2} t_{3} \tau_{3}^{2}}\left[(A-B)^{2}+p^{2}(A+B)^{2}\left(\frac{(\lambda-1)^{2}}{2 \lambda}+1\right)\right] .
$$

For the internal Einstein equations, only some components are non-trivial

$$
\begin{aligned}
& g_{s} T_{14}=\frac{1}{t_{1} t_{2} t_{3} \tau_{3}^{2}} \frac{p}{\sqrt{\lambda}}\left(A^{2}-B^{2}\right)(\lambda-1)\left(1-\gamma^{2}\right), \\
& g_{s} T_{23}=\frac{1}{t_{1} t_{2} t_{3} \tau_{3}^{2}} \frac{p}{\sqrt{\lambda}}\left(A^{2}-B^{2}\right)(\lambda-1)\left(1-\gamma^{2}\right), \\
& g_{s} T_{11}=\frac{1}{t_{1} t_{2} t_{3} \tau_{3}^{2}}\left[A^{2}-B^{2}+\frac{p^{2}}{\lambda}\left(A^{2}-B^{2} \lambda^{2}\right)-\gamma^{2}\left((A-B)^{2}+\frac{p^{2}}{\lambda}(A+B)^{2}\right)\right]-h^{2}+2 C, \\
& g_{s} T_{22}=\frac{1}{t_{1} t_{2} t_{3} \tau_{3}^{2}}\left[B^{2}-A^{2}+\frac{p^{2}}{\lambda}\left(B^{2} \lambda^{2}-A^{2}\right)-\gamma^{2}\left((A-B)^{2}+p^{2} \lambda(A+B)^{2}\right)\right]-h^{2}+2 C, \\
& g_{s} T_{33}=\frac{1}{t_{1} t_{2} t_{3} \tau_{3}^{2}}\left[B^{2}-A^{2}+\frac{p^{2}}{\lambda}\left(B^{2}-A^{2} \lambda^{2}\right)-\gamma^{2}\left((A-B)^{2}+\frac{p^{2}}{\lambda}(A+B)^{2}\right)\right]-h^{2}+2 C, \\
& g_{s} T_{44}=\frac{1}{t_{1} t_{2} t_{3} \tau_{3}^{2}}\left[A^{2}-B^{2}+\frac{p^{2}}{\lambda}\left(A^{2} \lambda^{2}-B^{2}\right)-\gamma^{2}\left((A-B)^{2}+p^{2} \lambda(A+B)^{2}\right)\right]-h^{2}+2 C, \\
& g_{s} T_{55}=-\frac{2}{t_{1} t_{2} t_{3} \tau_{3}^{2}}\left[(A-B)^{2}+p^{2}\left(\frac{\left(\lambda^{2}+1\right)}{2 \lambda}\left(A^{2}+B^{2}\right)+2 A B\right)\right]-2 h^{2}+2 C, \\
& g_{s} T_{66}=2 C .
\end{aligned}
$$

The remaining components set to zero the corresponding source term $T_{a b}=0$.

To solve these equations we need the explicit expressions for the source energy momentum tensor, (3.11). In six-dimensional frame indices we have

$$
\begin{aligned}
T_{a b} & =2 \kappa^{2} T_{p} \hat{*}\left\langle j, \delta_{c(a} e^{c} \otimes \iota_{b)} \operatorname{Im} X-\delta_{(a}^{c} \delta_{b) d} C_{c}^{d}\right\rangle \\
& =2 \kappa^{2} T_{p} \hat{*}\left(\sqrt{\left|g_{4}\right|} \mathrm{d}^{4} x \wedge\left\langle j, \delta_{c(a} e^{c} \otimes \iota_{b)} \operatorname{Im} X_{-}-\delta_{(a}^{c} \delta_{b) d} c_{c}^{d}\right\rangle\right) \\
& =2 \kappa^{2} T_{p} \frac{1}{\sqrt{\left|g_{6}\right|}}\left[j \wedge\left(\delta_{c(a} e^{c} \otimes \iota_{b)} \operatorname{Im} X_{3}-\left.\delta_{(a}^{c} \delta_{b) d} c_{c}^{d}\right|_{3}\right)\right]_{1 \ldots 6} \\
& =\frac{1}{\sqrt{\left|g_{6}\right|}}\left[\left(\mathrm{d} F_{2}-H F_{0}\right) \wedge\left(\delta_{c(a} e^{c} \otimes \iota_{b)} \operatorname{Im} X_{3}-\left.\delta_{(a}^{c} \delta_{b) d} c_{c}^{d}\right|_{3}\right)\right]_{1 \ldots 6} .
\end{aligned}
$$

Since, in our case, the source $j$ is a three-form,

$$
2 \kappa^{2} T_{p} j=\mathrm{d} F_{2}-H F_{0},
$$

only the three-form parts $\operatorname{Im} X_{3}$ and $\left.c_{c}^{d}\right|_{3}$ of $\operatorname{Im} X_{-}$and $c_{c}^{d}$ contribute to the equations. 
In the same way, we obtain

$$
g_{s} \tilde{T}_{0}=g_{s} 2 \kappa^{2} T_{p} \hat{*}\langle j, \operatorname{Im} X\rangle=\frac{1}{\sqrt{\left|g_{6}\right|}}\left[g_{s}\left(\mathrm{~d} F_{2}-H F_{0}\right) \wedge \operatorname{Im} X_{3}\right]_{1 \ldots 6} .
$$

Combining (3.3) and the explicit expression for $\mathrm{SU}(3)$ pure spinors, it is easy to see that $\operatorname{Im} X_{-}$decomposes into a one-form, a three-form and a five-form piece

$$
\operatorname{Im} X_{-}=\operatorname{Im} X_{1}+\operatorname{Im} X_{3}+\operatorname{Im} X_{5}
$$

where $^{20}$

$$
\begin{aligned}
\operatorname{Im} X_{1}= & \left(a_{k}^{i L}+a_{k}^{i R}\right) \mathrm{d} x^{k}-\left(a_{k}^{r L}-a_{k}^{r R}\right) g^{k j} \iota_{j} J+\left(g^{k m} g^{j l} \iota_{m} \iota_{l}\right)\left[-a_{k j}^{r} \operatorname{Re} \Omega+a_{k j}^{i} \operatorname{Im} \Omega\right] \\
\operatorname{Im} X_{3}= & -\left(a_{k}^{r L}+a_{k}^{r R}\right) \mathrm{d} x^{k} \wedge J-\left(a_{k}^{i L}-a_{k}^{i R}\right) g^{k j} \iota_{j} J \wedge J \\
& -\left[a_{0}^{r}-a_{k j}^{r}\left(g^{k j}-\left(g^{k l} \mathrm{~d} x^{j}+g^{j l} \mathrm{~d} x^{k}\right) \iota l\right)\right] \operatorname{Re} \Omega \\
& +\left[a_{0}^{i}-a_{k j}^{i}\left(g^{k j}-\left(g^{k l} \mathrm{~d} x^{j}+g^{j l} \mathrm{~d} x^{k}\right) \iota l\right)\right] \operatorname{Im} \Omega \\
\operatorname{Im} X_{5}= & \left.\left.-\frac{1}{2}\left[\left(a_{k}^{i L}+a_{k}^{i R}\right)\right) \mathrm{d} x^{k}-\left(a_{k}^{r L}-a_{k}^{r R}\right)\right) g^{k j} \iota_{j} J\right] \wedge J^{2} \\
& -\mathrm{d} x^{k} \wedge \mathrm{d} x^{j} \wedge\left[-a_{k j}^{r} \operatorname{Re} \Omega+a_{k j}^{i} \operatorname{Im} \Omega\right] .
\end{aligned}
$$

The superscripts $r$ and $i$ indicate real and imaginary parts:

$$
\begin{array}{ll}
a_{0}^{r}=\operatorname{Re}\left(\alpha_{0}-\widetilde{\alpha}_{0}\right), & a_{j k}^{r}=\operatorname{Re}\left(\alpha_{j k}-\widetilde{\alpha}_{j k}\right), \\
a_{0}^{i}=\operatorname{Im}\left(\alpha_{0}+\widetilde{\alpha}_{0}\right), & a_{j k}^{i}=\operatorname{Im}\left(\alpha_{j k}+\widetilde{\alpha}_{j k}\right) .
\end{array}
$$

and

$$
\begin{array}{rlrl}
a_{k}^{r L} & =\operatorname{Re}\left(\alpha_{k}^{L}-\widetilde{\alpha}_{k}^{L}\right), & a_{k}^{r R}=\operatorname{Re}\left(\alpha_{k}^{R}-\widetilde{\alpha}_{k}^{R}\right), \\
a_{k}^{i L}=\operatorname{Im}\left(\alpha_{k}^{L}+\widetilde{\alpha}_{k}^{L}\right), & a_{k}^{i R}=\operatorname{Im}\left(\alpha_{k}^{R}+\widetilde{\alpha}_{k}^{R}\right) .
\end{array}
$$

As already discussed, only the three-form parts of $\operatorname{Im} X_{-}$and $c_{c}^{d}$ contribute to the equations. Then, for simplicity, we choose to set to zero $\operatorname{Im} X_{1}$ and $\operatorname{Im} X_{5}$. This amounts to setting

$$
a_{k}^{r L}=a_{k}^{i L}=a_{k}^{r R}=a_{k}^{i R}=0,
$$

and choosing $a_{j k}^{r}$ and $a_{j k}^{i}$ symmetric. Then, in frame indices, $\operatorname{Im} X_{3}$ becomes

$$
\begin{aligned}
\operatorname{Im} X_{3}= & {\left[a_{0}^{i}-\operatorname{Tr}\left(a_{b c}^{i}\right)+a_{b c}^{i}\left(\delta^{b d} e^{c}+\delta^{c d} e^{b}\right) \iota_{d}\right] \operatorname{Im} \Omega } \\
& -\left[a_{0}^{r}-\operatorname{Tr}\left(a_{b c}^{r}\right)+a_{b c}^{r}\left(\delta^{b d} e^{c}+\delta^{c d} e^{b}\right) \iota_{d}\right] \operatorname{Re} \Omega .
\end{aligned}
$$

Similarly, we find that the three-form part of $c_{a}^{b}$ is given by

$$
\begin{aligned}
\left.c_{a}^{b}\right|_{3}= & 2 a_{a c}^{i}\left[-\delta^{b c}+\left(\delta^{c d} e^{b}+\delta^{b d} e^{c}\right) \iota_{d}\right] \operatorname{Im} \Omega \\
& -2 a_{a c}^{r}\left[-\delta^{b c}+\left(\delta^{c d} e^{b}+\delta^{b d} e^{c}\right) \iota_{d}\right] \operatorname{Re} \Omega .
\end{aligned}
$$

\footnotetext{
${ }^{20}$ We have not imposed (3.5) yet, and shall return to it later.
} 
The coefficients in $\operatorname{Im} X_{3}$ are free parameters which should be fixed by solving the dilaton and internal Einstein equations.

The equations $T_{m n}=0$ are satisfied by choosing ${ }^{21}$

$$
\begin{array}{rlrl}
a_{0}^{i} & =0 & a & =1, \ldots, 6, \\
a_{b c}^{i} & =0 & b, c & =1, \ldots, 6, \\
a_{b c}^{r} & =0 & (b c) & \notin\{(b b),(14),(23)\} .
\end{array}
$$

The Einstein and dilaton equations, (3.38) and (3.37) fix the other parameters

$$
\begin{aligned}
& a_{0}^{r}=-g_{s} \frac{\tilde{T}_{0}+T_{55}+T_{66}-x_{0}}{2\left(c_{1}+c_{2}\right)}, \\
& a_{14}^{r}=g_{s} \frac{T_{14}}{2\left(c_{2}-c_{1}\right)}, \\
& a_{23}^{r}=g_{s} \frac{T_{23}}{2\left(c_{1}-c_{2}\right)}, \\
& a_{11}^{r}=g_{s} \frac{1}{2\left(c_{2}-c_{1}\right)}\left[T_{11}-\frac{c_{2} \tilde{T}_{0}}{c_{1}+c_{2}}+\frac{x_{0} c_{1} c_{2}}{\left(c_{1}^{2}-c_{2}^{2}\right)}\right], \\
& a_{22}^{r}=g_{s} \frac{1}{2\left(c_{1}-c_{2}\right)}\left[T_{22}-\frac{c_{1} \tilde{T}_{0}}{c_{1}+c_{2}}+\frac{x_{0} c_{1} c_{2}}{\left(c_{2}^{2}-c_{1}^{2}\right)}\right], \\
& a_{33}^{r}=g_{s} \frac{1}{2\left(c_{1}-c_{2}\right)}\left[T_{33}-\frac{c_{1} \tilde{T}_{0}}{c_{1}+c_{2}}+\frac{x_{0} c_{1} c_{2}}{\left(c_{2}^{2}-c_{1}^{2}\right)}\right], \\
& a_{44}^{r}=g_{s} \frac{1}{2\left(c_{2}-c_{1}\right)}\left[T_{44}-\frac{c_{2} \tilde{T}_{0}}{c_{1}+c_{2}}+\frac{x_{0} c_{1} c_{2}}{\left(c_{1}^{2}-c_{2}^{2}\right)}\right], \\
& a_{55}^{r}=-g_{s} \frac{T_{55}}{2\left(c_{1}+c_{2}\right)}, \\
& a_{66}^{r}=g_{s} \frac{T_{66}-\tilde{T}_{0}}{2\left(c_{1}+c_{2}\right)},
\end{aligned}
$$

where $x_{0}=2 \tilde{T}_{0}-\left(T_{11}+T_{22}+T_{33}+T_{44}\right)$ and $T_{a b}$ are given by (3.38). The coefficients $c_{1}$ and $c_{2}$ appear in the source term of the Bianchi identity for $F_{2}$

$$
g_{s}\left(\mathrm{~d} F_{2}-H F_{0}\right)=c_{1} v^{1}+c_{2} v^{2}
$$

where $v^{1}$ and $v^{2}$ are covolumes of sources in the directions (146) and (236) and

$$
\begin{aligned}
& c_{1}=-\frac{h^{2}}{\gamma}+\frac{q_{1} q_{2}}{A t_{3}} \gamma\left[2(A-B)-p^{2} \frac{\lambda^{2}+1}{\lambda}(A+B)\right], \\
& c_{2}=-\frac{h^{2}}{\gamma}+\frac{q_{1} q_{2}}{B t_{3}} \gamma\left[2(B-A)-p^{2} \frac{\lambda^{2}+1}{\lambda}(A+B)\right] .
\end{aligned}
$$

\footnotetext{
${ }^{21}$ The parameters $a_{12}^{i}, a_{13}^{i}, a_{24}^{i}, a_{34}^{i}, a_{56}^{i}$ are not fixed by any equation. For simplicity, we decide to put them to zero.
} 
In agreement with our quantization conventions (see footnote 13), we impose that $\left(c_{1}+c_{2}\right)$ is an integer. We emphasize once more, that the overall tension of the intersecting sources is always negative (and so is $c_{1}+c_{2}$ ), but depending on the parameters of the solution the individual sources may be either O6 planes or D6 branes.

So far, we have solved the external and internal Einstein equations, the dilaton equation of motion, and checked that the Bianchi identity for $F_{2}$ is satisfied. As far as the bulk fields are concerned, we should also solve the equations of motion and the remaining Bianchi identities for the fluxes. These are actually automatically satisfied by our ansatz for the fluxes, provided $j \wedge \operatorname{Im} X_{1}=0$. As a matter of fact, our choice of the parameters $a$ in (3.49) already sets $\operatorname{Im} X_{1}$ to zero, so we are done with the bulk fields.

As a last step in the construction of a de Sitter solution (we recall we mean here a solution to the equations derived from our proposed action for the sources), we need to check the source fields equations of motion. One should vary our source action with respect to the world-volume coordinates and the gauge fields. The latter is trivially satisfied, since we do not consider any gauge field here, and the pullback of the $B$-field giving (3.29) vanishes. For the world-volume coordinates, from our action $-T_{p} \int_{\Sigma} e^{-\phi} i^{*}[\operatorname{Im} X]$ and WZ, one can derive, as discussed in the Introduction, an equation of motion of the form

$$
\partial_{\left[i_{1}\right.}\left(e^{-\phi} \operatorname{Im} X_{3}\right)_{\left.i_{2} i_{3}\right] \alpha} \sim\left(* F_{2}\right)_{\left[i_{1} i_{2} i_{3}\right] \alpha},
$$

where $i_{k}$ label world-volume directions, and $\alpha$ is orthogonal. One can check that pulling back any three indices of the four-form $* F_{2}$ to the world-volume gives zero, as discussed after (1.23). The left-hand side also vanishes (see (3.55)), and so we conclude that the world-volume equations of motion are satisfied.

This concludes our resolution of all equations of motion derived from the action (3.7) which contains our proposal for sources breaking bulk supersymmetry. Provided one chooses the free parameters as discussed below (3.35), one can obtain a de Sitter solution. In the next section, we come back to the question of generalizing first order differential equations to the non-supersymmetric case. This will fix for us the free parameters to values which indeed give a de Sitter solution. In section 3.3 we will argue that the solution we found here is also a solution to the equations derived with the standard source action.

\subsection{More on the polyform $X$}

In this section, we will try to provide further justification for our choice of polyform $X_{-}$.

In supersymmetric compactifications, the imaginary part of the non-closed pure spinor, $\Phi_{-}$in type IIA, on one side, defines the calibration for the sources and, on the other, gives the bulk RR fields in the supersymmetry equations (1.1). We will show that, for our de Sitter solution, the polyform $X_{-}$satisfies the same equations $\Phi_{-}$satisfies in the supersymmetric case

$$
\begin{aligned}
& (\mathrm{d}-H) \operatorname{Re} X_{-}=0, \\
& (\mathrm{~d}-H) \operatorname{Im} X_{-}=c_{0} g_{s} * \lambda(F),
\end{aligned}
$$

where the constant $c_{0}$ can a priori be different from 1 . 
Keeping only the parameters $a$ that are non-zero in the de Sitter solution (3.50), it is easy to compute

$$
\begin{aligned}
\mathrm{d}\left(\operatorname{Im} X_{-}\right)= & {\left[( a _ { 0 } ^ { r } + a _ { 6 6 } ^ { r } - a _ { 5 5 } ^ { r } ) \left[p\left(q_{1}+q_{2}\right)\left(e^{1} \wedge e^{3}+e^{2} \wedge e^{4}\right)\right.\right.} \\
& \left.-\left(q_{1}-q_{2}\right)\left(e^{1} \wedge e^{2}-e^{3} \wedge e^{4}\right)\right] \wedge e^{5} \wedge e^{6} \\
& -\left(a_{11}^{r}+a_{44}^{r}-a_{22}^{r}-a_{33}^{r}\right)\left[p\left(q_{1}-q_{2}\right)\left(e^{1} \wedge e^{3}+e^{2} \wedge e^{4}\right)\right. \\
& \left.-\left(q_{1}+q_{2}\right)\left(e^{1} \wedge e^{2}-e^{3} \wedge e^{4}\right)\right] \wedge e^{5} \wedge e^{6},
\end{aligned}
$$

and

$$
H \wedge \operatorname{Im} X_{-}=-2 h\left(a_{0}^{r}+a_{66}^{r}-a_{55}^{r}\right) e^{1} \wedge e^{2} \wedge e^{3} \wedge e^{4} \wedge e^{5} \wedge e^{6} .
$$

In order to have $\mathrm{d}\left(\operatorname{Im} X_{-}\right)$proportional to $g_{s} * F_{2}$, one must impose the relation

$$
a_{11}^{r}+a_{44}^{r}-a_{22}^{r}-a_{33}^{r}=0 .
$$

Then, one has

$$
\begin{aligned}
\mathrm{d}\left(\operatorname{Im} X_{-}\right) & =-c_{0} g_{s} * F_{2}, \\
H \wedge \operatorname{Im} X_{-} & =-2 \gamma^{2} c_{0} g_{s} * F_{0},
\end{aligned}
$$

with

$$
c_{0}=\frac{a_{0}^{r}+a_{66}^{r}-a_{55}^{r}}{\gamma}=-g_{s} \frac{\tilde{T}_{0}}{\gamma\left(c_{1}+c_{2}\right)} .
$$

To obtain the second equality, we used the explicit expression (3.50), (3.38) for the parameters $a$, while $c_{1}$ and $c_{2}$ are defined in (3.52). Also, using (3.50), it is easy to show that the constraint (3.57) reduces to

$$
x_{0}=2 \tilde{T}_{0}-\left(T_{11}+T_{22}+T_{33}+T_{44}\right)=0 \quad \Leftrightarrow \quad\left(2 \gamma^{2}-1\right) h^{2}=0 .
$$

Therefore, for $^{22}$

$$
\gamma^{2}=\frac{1}{2}
$$

we can write a differential equation for $\operatorname{Im} X_{-}$

$$
(\mathrm{d}-H) \operatorname{Im} X_{-}=c_{0} g_{s} * \lambda(F),
$$

which is the analogue of the supersymmetry equations ${ }^{23}$ for $\operatorname{Im} \Phi_{-}$. In addition, fixing the value $\gamma^{2}=1 / 2$ gives a de Sitter solution, according to the condition (3.35).

\footnotetext{
${ }^{22}$ Clearly also $h=0$ (no NS flux) is a solution to this constraint. It would be interesting to explore the possibility of having de Sitter or non-supersymmetric Minkowski solution with $h=0$. Notice that, in this case, the condition of having $F_{0} \neq 0$ [16], necessary to avoid de Sitter no-go theorems [14], is not required.

${ }^{23}$ Notice that from the equation for $\operatorname{Im} X_{-}$we recover the condition $T_{0}>0$ (3.19). Indeed, as in [41],
} starting from (3.41) we have

$$
\frac{T_{0}}{p+1} \int_{M} \operatorname{vol}_{(6)}=-\int_{M}\left\langle\mathrm{~d}_{H} F, \operatorname{Im} X_{-}\right\rangle=-\int_{M}\left\langle F, \mathrm{~d}_{H} \operatorname{Im} X_{-}\right\rangle=c_{0} g_{s} \int_{M}\langle * \lambda(F), F\rangle>0 .
$$


The value of the constant $c_{0}$ is also fixed by the solution. Indeed, in order for $X_{-}$ to reproduce the correct Born-Infeld action (3.5) on-shell, we get from our solution that a combination of coefficients of $X_{-}$has to be one: $a_{0}^{r}+a_{66}^{r}-a_{55}^{r}=1$. Out of (3.59), we deduce that we have to impose $c_{0} \gamma=1$. This relation is automatically satisfied for supersymmetric backgrounds, where $c_{0}=\gamma=1$ and the pullback of Re $\Omega$ agrees with the DBI action on the solution. In our non-supersymmetric solution, the condition $c_{0} \gamma=1$ fixes the value of the constant, $c_{0}=\sqrt{2}$.

More generally, requiring the two actions being equal on-shell can be formulated as $-g_{s} \tilde{T}_{0}=c_{1}+c_{2}$, where the right-hand side is given by the sum of the source charges. Indeed, as we can see in (3.41), if $\operatorname{Im} X$ gives the sum of the source volume forms on-shell, and $j$ or $\mathrm{d}_{H} F$ gives the sum of the charges times the covolumes (Bianchi identity), then $\tilde{T}_{0}$ should be given by the sum of the charges; this sum is negative, hence the minus sign. We can verify that this condition is equivalent for our solution to the condition $c_{0} \gamma=1$, given the second equality in (3.59). Finally, let us note that such a relation would fix one of the three parameters $h, \gamma, \lambda$ in terms of the others and the moduli. In particular, for $\lambda=1$, one gets

$$
h^{2}=\frac{(A-B)^{2}+p^{2}(A+B)^{2}}{t_{1} t_{2} t_{3} \tau_{3}^{2}} \frac{(\gamma-1)(1-2 \gamma) \gamma^{2}}{\gamma^{2}-3 \gamma+1} .
$$

Note one clearly recovers the supersymmetric case with $\gamma=1$. For our de Sitter solution, one should impose instead $\gamma=\frac{1}{\sqrt{2}}$, and then $h \neq 0$.

We can now show that $\mathrm{d}_{H}$ - closure can be imposed on $\operatorname{Re} X_{-}$. Indeed, the three-form part of $\operatorname{Re} X_{-}$can be written as

$$
\begin{aligned}
\operatorname{Re} X_{3}= & -\left[b_{0}^{r}-\operatorname{Tr}\left(b_{k j}^{r}\right)+b_{k j}^{r}\left(g^{k l} d x^{j}+g^{j l} d x^{k}\right) \iota_{l}\right] \operatorname{Re} \Omega \\
& +\left[b_{0}^{i}-\operatorname{Tr}\left(b_{k j}^{i}\right)+b_{k j}^{i}\left(g^{k l} d x^{j}+g^{j l} d x^{k}\right) \iota_{l}\right] \operatorname{Im} \Omega \\
& +\left[\left(b_{k}^{i R}-b_{k}^{i L}\right) d x^{k}+g^{k l}\left(b_{k}^{r R}-b_{k}^{r L}\right) \iota_{l} J\right] \wedge J,
\end{aligned}
$$

where, as for $\operatorname{Im} X_{3}$, we have defined

$$
\begin{aligned}
b_{0}^{r} & =\operatorname{Im}\left(\widetilde{\alpha}_{0}-\alpha_{0}\right) & b_{k j}^{r} & =\operatorname{Im}\left(\widetilde{\alpha}_{k j}-\alpha_{k j}\right), \\
b_{0}^{i} & =\operatorname{Re}\left(\widetilde{\alpha}_{0}+\alpha_{0}\right) & b_{k j}^{i} & =\operatorname{Re}\left(\widetilde{\alpha}_{k j}+\alpha_{k j}\right), \\
b_{k}^{r L} & =\operatorname{Re}\left(\widetilde{\alpha}_{k}^{L}+\alpha_{k}^{L}\right) & b_{k}^{r R} & =\operatorname{Re}\left(\alpha_{k}^{R}+\widetilde{\alpha}_{k}^{R}\right), \\
b_{k}^{i L} & =\operatorname{Im}\left(\widetilde{\alpha}_{k}^{L}-\alpha_{k}^{L}\right) & b_{k}^{i R} & =\operatorname{Im}\left(\alpha_{k}^{R}-\widetilde{\alpha}_{k}^{R}\right) .
\end{aligned}
$$

Consistently with (3.49), we can choose

$$
\begin{array}{rlrl}
b_{0}^{r} & =0, \\
b_{k}^{r L} & =b_{k}^{r L}=b_{k}^{i L}=b_{k}^{i R}=0 & & \\
b_{j k}^{r} & =0 & & \forall k=1, \ldots 6 \\
b_{j k}^{i} & =0 \quad \text { for }(k j) \notin\{(k k),(14),(23),(41),(32)\} .
\end{array}
$$

Furthermore, choosing

$$
\frac{b_{14}^{i}}{t_{1}}=-\frac{b_{23}^{i}}{t_{2} \tau_{3}^{2}}, \quad \frac{b_{11}^{i}}{t_{1}}+\frac{b_{33}^{i}}{t_{2} \tau_{3}^{2}}-\frac{b_{22}^{i}}{t_{2} \tau_{3}^{2} \lambda}-\frac{b_{44}^{i}}{t_{1} \lambda}=0,
$$


we obtain

$$
\mathrm{d}_{H}\left(\operatorname{Re} X_{3}\right)=\sqrt{t_{1} t_{2} t_{3}} \tau_{3} \tau_{6} p(1-\lambda)\left(b_{0}^{i}+\frac{b_{66}^{i}}{t_{3} \tau_{6}^{2}}-\frac{b_{55}^{i}}{t_{3}}\right)\left(q_{2} e^{1} \wedge e^{4}+q_{1} e^{2} \wedge e^{3}\right) \wedge e^{5} \wedge e^{6},
$$

which is zero either in the SUSY solution, or by further setting

$$
b_{0}^{i}=-\frac{b_{66}^{i}}{t_{3} \tau_{6}^{2}}+\frac{b_{55}^{i}}{t_{3}}
$$

While these equations are derived in the vanishing warp factor and constant dilaton limit, their extension to the general case is natural ${ }^{24}$

$$
\begin{aligned}
& \mathrm{d}_{H}\left(e^{2 A-\phi} \operatorname{Re} X_{-}\right)=0, \\
& \mathrm{~d}_{H}\left(e^{4 A-\phi} \operatorname{Im} X_{-}\right)=c_{0} e^{4 A} * \lambda(F) .
\end{aligned}
$$

In general the odd form $X_{-}$should receive contribution from both pure spinors, but in our solution we have chosen to "decouple" the even pure spinor completely. Note that any two objects in the trio of the even and odd compatible pure spinors and the metric determine the third. Here we have worked with the almost complex structure and the metric. In the supersymmetric backgrounds it is clearly more convenient to solve the first order equations for the pure spinors rather than the Einstein equation for the metric. Hence it is natural to ask if and when it might be possible to find an even-form counterpart to (3.70), $X_{+}$, so that $X_{-}$and $X_{+}$(together with flux Bianchi identities) imply the solution to the Einstein equations. However it is not yet clear to us what the correct generalization of the notion of compatibility is, and what algebraic properties $X_{+}$should satisfy. Hoping for a symmetry with the supersymmetric solutions (and the possibility of having a solution to some variational problem) one may construct $X_{+}$satisfying

$$
\mathrm{d}_{H}\left(e^{3 A-\phi} X_{+}\right)=0
$$

Assuming $X_{+}$has an expansion similar to that of $X_{-}$, which does not receive contributions from $\Omega$, this amounts to finding a closed two-form on $\mathfrak{g}_{5.17}^{p,-p, \pm 1} \times S^{1}$. It is indeed not hard to construct such a form for our solution, since the symplectic form itself is closed, provided $\tau_{2}=0$ (even if $\lambda \neq 1$, see (2.75)). Even if we do not take $\tau_{2}=0$, finding a conformally closed $X_{+}$of this form is always possible, since the manifold is symplectic. A better understanding of such first order equations applicable to non-supersymmetric backgrounds is a work in progress and we hope to return to it in a future publication.

\footnotetext{
${ }^{24}$ Just like $\Phi_{-}, X_{-}$is globally defined, and both $B$-field and the dilaton are needed in order to define an isomorphism between such forms and the positive and negative helicity spin bundles $S^{ \pm}(E)$ [43]. The dilaton assures the correct transformation under $G L(6)$, making the (non-pure) spinor $e^{-\phi} e^{-B} X_{-}$the natural variable for the first order equations (3.70).
} 


\subsection{A solution for the standard source action?}

In this paper we made a proposal of an action for sources breaking bulk supersymmetry. As discussed in the Introduction and at the beginning of section 3, we cannot conclude (as one would do in the supersymmetric case) whether the equations of motion derived from the action (3.7) are the same as those derived from the standard source action DBI + WZ. Our proposal is to be considered as an assumption with interesting consequences, we are not able to prove such an equivalence, but hope to provide a better justification of it in future work. What can be done is to verify that the solution found in our example is indeed a solution to the equations of motion derived from the standard source action. Let us discuss now in practice what should be checked, starting with the world-volume equations of motion.

There are two equations to consider, coming from the variation of DBI $+\mathrm{WZ}$ action with respect to the world-volume coordinates and the gauge fields (for a general form of these equations see [50]). The latter is easier, and we shall consider it first. In our solution the dilaton is constant and the world-volume gauge fields vanish. Moreover we recall that the pullback of the $B$-field computed from (3.29) also vanishes. Then the equation reads

$$
\partial_{i}\left(e^{-\phi} \sqrt{\left|i^{*}[g]\right|}\left(i^{*}[g]\right)^{[i j]}\right) \sim \epsilon^{j k l}\left(i^{*}\left[* F_{4}\right]\right)_{k l},
$$

where $i, j, k, l$ are indices along the brane world-volume. Since our solution has no RR fourform flux, both sides vanish trivially. The variation of the world-volume action with respect to the world-volume coordinates (again, in presence of constant dilaton and vanishing pullback of $B$ ) connects the trace of the second fundamental form $\mathcal{S}_{i j}^{\alpha}$ to the RR fluxes $(\alpha$ spans normal directions). It reads

$$
e^{-\phi}\left(i^{*}[g]\right)^{i j} \mathcal{S}_{i j}^{\alpha} \sim \epsilon^{j k l}\left(* F_{2}\right)_{j k l}^{\alpha} .
$$

One can check that pulling back any three indices of the four-form $* F_{2}$ to the worldvolume gives zero. ${ }^{25}$ For our intersecting configuration, we need to worry only about $\alpha=5$, and may use the relation of the second fundamental form with the (components of) the spin connection $\omega_{i}^{\alpha}=\mathcal{S}_{i j}^{\alpha} e^{j}$. We can check that while the second fundamental form does not vanish (the embedding is not geodesic), it has no diagonal element. However the metric (3.28) in the basis (2.72) is diagonal, and $\left(i^{*}[g]\right)^{i j} \mathcal{S}_{i j}^{\alpha}$ vanishes. Thus the worldvolume equations of motion are satisfied.

Let us now consider the bulk field equations of motion. As mentioned at the end of section 3.1.1, the ansatz chosen for the fluxes guarantees that their equations of motion and Bianchi identities are satisfied. Let us also emphasize the following details: first we do not have any $B$-field along the sources and therefore a correction term due to the source in its equation of motion could be discarded; second the proposed generalization of the first order equations (3.70), satisfied by our solution, guarantees that the RR equations of motion are satisfied. Therefore, for the bulk fields, only the internal Einstein equation and the dilaton equation of motion remain to be checked.

\footnotetext{
${ }^{25}$ This check is analoguous to that of the corresponding equation of motion derived from our proposed source action, as discussed at the end of section 3.1.1.
} 
The dependence of the dilaton equation on the source action is simply through $\tilde{T}_{0}$ (see for instance (3.19)), which is proportional to the source action on-shell. Therefore, as long as the standard source action and our proposed action match on-shell, the dilaton equations of motion are the same. As discussed in the previous section, this equality amounts in general to the condition $-g_{s} \tilde{T}_{0}=c_{1}+c_{2}$, which for our solution is equivalent to $c_{0} \gamma=1$. This fixes one of the three parameters $h, \gamma, \lambda$ in terms of the others and the moduli (see for instance (3.63)). Provided this condition is enforced, the dilaton equation of motion derived from DBI is therefore satisfied by our solution.

We are now left with the internal Einstein equation. An explicit check can be done for the family given by:

$$
\lambda=1 \quad F_{0} \neq 0, h \neq 0, \text { given by }(3.63),
$$

with particular interest in the non-supersymmetric value $\gamma=\frac{1}{\sqrt{2}}$ giving our de Sitter solution. Solving the Einstein equation amounts to match the values of the energy-momentum tensor $T_{a b}$ given by (3.38). In the supersymmetric case, one can derive from the standard source action that the non-zero components of $T_{a b}$ of one source are the diagonal ones along the source directions, and are all equal. We recover this situation in the family we consider by simply taking $\gamma=1$. For our non-supersymmetric solution, the supersymmetry breaking will manifest itself as $T_{55} \neq 0$ and $T_{66} \neq T_{11}+T_{22}$. Then, in order to match the results, one needs to consider a non-trivial dependence of the embedding functions on the metric moduli. The computation is rather involved and not particularly enlightening, thus we will not present it here. However, let us note that this non standard embedding corresponds to our interpretation of the proposed action, as discussed in the Introduction. We can also obtain a perturbative solution (the perturbation parameter is $\epsilon=\lambda-1$ ) where the deviation from the SUSY solution is more severe due to $T_{14}$ and $T_{23}$ not being zero as opposed to their supersymmetric value.

Let us end this section by adding few words about the stability of our solution. Solving all the equations of motion of course means extremizing the energy density of the bulk plus brane system, but we cannot be sure that the solution is a minimum for arbitrary values of the parameters. The problem is currently under study. For the time being we can try to give some heuristic justification of the fact we believe our non-supersymmetric solution is stable. For $\lambda=1$ and $\gamma=1$ the manifold admits the supersymmetric solution described in section 2.3. By keeping $\lambda=1$ and setting $\gamma=1 / \sqrt{2}$ we obtain a non-supersymmetric solution with the same internal geometry as in the SUSY case, meaning the metric is not changed and the directions wrapped are the same. The pullback of $\operatorname{Im} X_{-}$does coincide with the pullback of the (generalized) calibrating form Re $\Omega$. In a sense the brane is still wrapping a minimal volume cycle (even if this is done with a different embedding), and we can imagine the parameters, other than $\gamma$, can be chosen in such a way to have small contributions to the potential from the supersymmetry breaking term, and the energy density of combined bulk and brane system at the minimum. 


\subsection{Four-dimensional analysis}

In this section we do a partial study of the stability of our solution by analyzing the four dimensional effective potential with respect to two moduli.

The search for de Sitter vacua, or for no-go theorems against their existence, has generally been performed from a four-dimensional point of view [13-19, 24, 25], analysing the behaviour of the four dimensional effective potential with respect to its moduli dependence. In this section, we want to make contact with this approach and show that our solution has the good behaviour one expects to find for de Sitter vacua, as far as the volume and the dilaton are concerned. We use in this section the ten-dimensional action (3.7) which contains our proposal for sources breaking bulk supersymmetry. We will show that this proposal gives rise to interesting new terms in the potential.

\subsubsection{Moduli and $4 d$ Einstein frame}

Let us consider the ten-dimensional action (3.7). By Kaluza-Klein reduction on the internal manifold, we obtain a four-dimensional effective action for the moduli. In particular, in addition to the kinetic terms, the four-dimensional action will contain a potential for the moduli fields. Their number and the way they enter the potential will depend on the peculiar features of the single model.

A de Sitter solution of the four-dimensional effective action will correspond to a positive valued minimum of the potential. Determining the minima of the potential is in general rather difficult, since, a priori one should extremize along all the directions in the moduli space. This complicated problem is generally solved only by numerical analysis, because of the large number of variables. However, some information can be extracted by restricting the analysis to a subset of the moduli fields.

For whatever choice of the manifold on which the compactification is performed, we are always able to isolate two universal moduli: the internal volume and the four-dimensional dilaton. Their appearance in the effective potential at tree-level is also universal. We will then only focus on these two moduli. We define the internal volume as

$$
\int_{M} \mathrm{~d}^{6} x \sqrt{\left|g_{6}\right|}=\frac{L^{6}}{2}=\frac{L_{0}^{6}}{2} \rho^{3},
$$

where the factor of $\frac{1}{2}$ is due to the orientifold and the vacuum value is $\rho=1$. Defining the ten-dimensional dilaton fluctuation as $e^{-\tilde{\phi}}=g_{s} e^{-\phi}$, the four-dimensional dilaton is given by

$$
\sigma=\rho^{\frac{3}{2}} e^{-\tilde{\phi}}
$$

Then reducing the action (3.7), we obtain the four-dimensional effective action for gravity, $4 d$ dilaton and volume modulus in the string frame

$$
S=\frac{1}{2 \kappa^{2}} \int \mathrm{d}^{4} x \sqrt{\left|g_{4}\right|}\left[\frac{L^{6}}{2} e^{-2 \phi}\left(R_{4}+4|\nabla \phi|^{2}\right)-2 \kappa^{2} U\right],
$$

with $U(\rho, \sigma)$ the four-dimensional potential. To derive the explicit form of the potential, we need to determine how the internal Ricci scalar, fluxes and source terms scale with the 
volume. For $R_{6}$ and the fluxes this is easily computed

$$
R_{6} \rightarrow \rho^{-1} R_{6}, \quad|H|^{2} \rightarrow \rho^{-3}|H|^{2}, \quad\left|F_{k}\right|^{2} \rightarrow \rho^{-k}\left|F_{k}\right|^{2} .
$$

The source term requires some more attention. As shown in (3.41),

$$
2 \kappa^{2} T_{p} \hat{*}\langle j, \operatorname{Im} X\rangle=\frac{\left[\left(\mathrm{d} F_{2}-H F_{0}\right) \wedge \operatorname{Im} X_{3}\right]_{1 \ldots 6}}{\sqrt{\left|g_{6}\right|}} .
$$

The terms in $\operatorname{Im} X_{3}$ in (3.43) appearing with $a_{0}, a_{j k}$ and $a_{k}^{(L, R)}$ scale differently with the volume. Let us denote them by $X_{0}, X_{\Omega}$ and $X_{J}$, respectively

$$
\operatorname{Im} X_{3}=X_{0}+X_{\Omega}+X_{J} .
$$

Their $\rho$ dependence is determined by the scaling of the forms $J$ and $\Omega$

$$
J \rightarrow \rho J, \Omega \rightarrow \rho^{\frac{3}{2}} \Omega,
$$

and by the metric factors in the gamma matrices of (3.43)

$$
X_{0} \rightarrow \rho^{\frac{3}{2}} X_{0}, X_{\Omega} \rightarrow \rho^{\frac{1}{2}} X_{\Omega}, X_{J} \rightarrow \rho X_{J} .
$$

Then, the source term scales as

$$
\frac{\left[\left(\mathrm{d} F_{2}-H F_{0}\right) \wedge \operatorname{Im} X_{3}\right]_{1 \ldots 6}}{\sqrt{\left|g_{6}\right|}} \rightarrow \rho^{-\frac{3}{2}}\left(b_{0}+b_{1} \rho^{-1}+b_{2} \rho^{-\frac{1}{2}}\right),
$$

where

$$
\begin{aligned}
& b_{0}=\frac{\left[\left(\mathrm{d} F_{2}-H F_{0}\right) \wedge X_{0}\right]_{1 \ldots 6}}{\sqrt{\left|g_{6}\right|}}, \\
& b_{1}=\frac{\left[\left(\mathrm{d} F_{2}-H F_{0}\right) \wedge X_{\Omega}\right]_{1 \ldots 6}}{\sqrt{\left|g_{6}\right|}}, \\
& b_{2}=\frac{\left[\left(\mathrm{d} F_{2}-H F_{0}\right) \wedge X_{J}\right]_{1 \ldots 6}}{\sqrt{\left|g_{6}\right|}},
\end{aligned}
$$

are vacuum values. Then the four-dimensional potential for $\rho$ and $\sigma$ becomes

$$
\begin{aligned}
U & =\frac{1}{2 \kappa^{2}} \int_{M} \mathrm{~d}^{6} x \sqrt{\left|g_{6}\right|}\left[e^{-2 \phi}\left(-R_{6}+\frac{1}{2}|H|^{2}\right)+\frac{1}{2}\left(\left|F_{0}\right|^{2}+\left|F_{2}\right|^{2}\right)-2 \kappa^{2} T_{p} e^{-\phi} \hat{*}\langle j, \operatorname{Im} X\rangle\right] \\
& =\frac{L_{0}^{6}}{4 g_{s}^{2} \kappa^{2}} \sigma^{2}\left[\left(-\frac{R_{6}}{\rho}+\frac{|H|^{2}}{2 \rho^{3}}\right)-\frac{g_{s}}{\sigma}\left(b_{0}+\frac{b_{1}}{\rho}+\frac{b_{2}}{\sqrt{\rho}}\right)+\frac{g_{s}^{2} \rho^{3}}{2 \sigma^{2}}\left(\left|F_{0}\right|^{2}+\frac{\left|F_{2}\right|^{2}}{\rho^{2}}\right)\right] \cdot(3.85)
\end{aligned}
$$

Note that the terms in $b_{1}$ and $b_{2}$ are purely non-supersymmetric contributions of the source. They are due to the new metric dependence of the source action with respect to the supersymmetric case.

In order to correctly identify the cosmological constant, but also to perform the study of the moduli dependence, we need to go to the four-dimensional Einstein frame

$$
g_{\mu \nu E}=\sigma^{2} g_{\mu \nu} .
$$


The four-dimensional Einstein-Hilbert term transforms as ${ }^{26}$

$$
\begin{aligned}
\frac{1}{2 \kappa^{2}} \int \mathrm{d}^{4} x \sqrt{\left|g_{4}\right|} \frac{L^{6}}{2} e^{-2 \phi} R_{4} & =\frac{L_{0}^{6}}{2 g_{s}^{2} 2 \kappa^{2}} \int \mathrm{d}^{4} x \sqrt{\left|g_{4}\right|} \sigma^{2} R_{4} \\
& =M_{4}^{2} \int \mathrm{d}^{4} x \sqrt{\left|g_{4 E}\right|} R_{4 E}
\end{aligned}
$$

where we denote Einstein frame quantities by $E$, and we introduced $M_{4}^{2}=\frac{L_{0}^{6}}{2 g_{s}^{2} 2 \kappa^{2}}$, the squared four-dimensional Planck mass. Similarly, the four-dimensional potential in the Einstein frame becomes

$$
U_{E}=\sigma^{-4} U=4 \kappa^{4} M_{4}^{4} \frac{e^{4 \phi}}{\left(\frac{L^{6}}{2}\right)^{2}} U
$$

and we can write the Einstein frame action as

$$
S=M_{4}^{2} \int \mathrm{d}^{4} x \sqrt{\left|g_{4 E}\right|}\left(R_{4 E}+\operatorname{kin}-\frac{1}{M_{4}^{2}} U_{E}\right) .
$$

The cosmological constant, (3.15), is then related to the vacuum value of the potential

$$
\Lambda=\left.\frac{1}{2 M_{4}^{2}} U_{E}\right|_{0} .
$$

\subsubsection{Extremization and stability}

In order to find a solution, one should determine the minima of the potential. For our choice of moduli, $\rho$ and $\sigma$, one has

$$
\begin{aligned}
& \frac{\partial U_{E}}{\partial \sigma}=-\frac{M_{4}^{2}}{\sigma^{5}}\left[2 g_{s}^{2}\left(\left|F_{0}\right|^{2} \rho^{3}+\left|F_{2}\right|^{2} \rho\right)+2 \sigma^{2}\left(-\frac{R_{6}}{\rho}+\frac{|H|^{2}}{2 \rho^{3}}\right)-3 \sigma g_{s}\left(b_{0}+\frac{b_{1}}{\rho}+\frac{b_{2}}{\sqrt{\rho}}\right)\right],( \\
& \frac{\partial U_{E}}{\partial \rho}=\frac{M_{4}^{2}}{\sigma^{2}}\left[\left(\frac{R_{6}}{\rho^{2}}-\frac{3|H|^{2}}{2 \rho^{4}}\right)+\frac{g_{s}}{\sigma}\left(\frac{b_{1}}{\rho^{2}}+\frac{b_{2}}{2 \sqrt{\rho^{3}}}\right)+\frac{g_{s}^{2}}{2 \sigma^{2}}\left(3\left|F_{0}\right|^{2} \rho^{2}+\left|F_{2}\right|^{2}\right)\right] .
\end{aligned}
$$

In our conventions, the extremization conditions are

$$
\left.\frac{\partial U_{E}}{\partial \sigma}\right|_{\sigma=\rho=1}=0 \quad,\left.\quad \frac{\partial U_{E}}{\partial \rho}\right|_{\sigma=\rho=1}=0,
$$

where $\sigma=\rho=1$ are the values of the moduli on the vacuum. Actually, the conditions (3.93) are equivalent to the ten-dimensional dilaton e.o.m. and the trace of internal Einstein equation. Combining the dilaton equation (3.19) and the trace of the internal Einstein equation, (3.18), we can write the six-dimensional Ricci scalar as

$$
R_{6}=\frac{3}{2}|H|^{2}-\frac{g_{s}^{2}}{2}\left(3\left|F_{0}\right|^{2}+\left|F_{2}\right|^{2}\right)-\frac{g_{s}}{2}\left(T_{0}-T\right),
$$

\footnotetext{
${ }^{26}$ Under a conformal rescaling of the four dimensional metric we have

$$
g_{\mu \nu} \rightarrow e^{2 \lambda} g_{\mu \nu} \quad \Rightarrow \quad \sqrt{\left|g_{4}\right|} \rightarrow e^{4 \lambda} \sqrt{\left|g_{4}\right|}, R_{4} \rightarrow e^{-2 \lambda} R_{4} .
$$
}


where

$$
\begin{aligned}
T_{0}-T & =2 \kappa^{2} T_{p} \hat{*}\left\langle j, C_{m}^{m}\right\rangle=\frac{\left[\left(\mathrm{d} F_{2}-H F_{0}\right) \wedge\left(X_{J}+2 X_{\Omega}\right)\right]_{1 \ldots 6}}{\sqrt{\left|g_{6}\right|}} \\
& =2 b_{1}+b_{2} .
\end{aligned}
$$

In the last line we used (3.84). With this expression for $T_{0}-T$, it is immediate to verify that (3.94) is indeed equal to the $\partial_{\rho} U_{E}$ in (3.93). Similarly, one can see that using (3.92), (3.83), (3.41) and (3.93), the dilaton equation (3.19) reduces to $\partial_{\sigma} U_{E}$ in (3.93).

From the equivalence of the ten-dimensional equations and (3.93) we see that the tendimensional solution discussed in the previous sections does indeed satisfy the extremization conditions (3.93). The next step is to see whether such extremum correspond to a minimum of the potential and whether, furthermore, it is stable.

Let us consider (3.92) and discuss the $\rho$ dependence of the potential. It is convenient to define the function

$$
P\left(\rho^{2}\right)=\frac{\partial U_{E}}{\partial \rho} \frac{\sigma^{2} \rho^{4}}{M_{4}^{2}}
$$

It is easy to check that $P\left(\rho^{2}\right)$ is negative for $\rho=0$ and positive for $\rho \rightarrow \infty$. Hence there must be a real positive root and this is a minimum of $U_{E}$. A priori, $P\left(\rho^{2}\right)$ could have other zeros. Let us focus only on the situation in which $b_{2}=0$, which, in particular, is the case for our ten-dimensional solution. In that case, $P\left(\rho^{2}\right)$ has two other roots which are either complex conjugate, ${ }^{27}$ or real and negative, according to the value of the parameters. Indeed, studying $\partial_{\rho^{2}} P$, one can show that $P\left(\rho^{2}\right)$ can be 0 only once. Therefore, at least for $b_{2}=0$, there is only one extremum of $U_{E}$ in $\rho$ and it is a minimum. So satisfying the extremization in $\rho$ is enough for the stability.

Let us now analyze the $\sigma$ dependence of (3.88). It is easy to see that the potential admits an extremum for

$$
\sigma_{ \pm}=\frac{1}{4 a}\left(3 b \pm \sqrt{8 b^{2}\left(\frac{9}{8}-\frac{4 a c}{b^{2}}\right)}\right) \quad \frac{4 a c}{b^{2}}<\frac{9}{8}
$$

where for simplicity we introduced

$$
\begin{aligned}
& a=-R_{6} \rho^{-1}+\frac{1}{2}|H|^{2} \rho^{-3}, \\
& b=g_{s}\left(b_{0}+b_{1} \rho^{-1}+b_{2} \rho^{-\frac{1}{2}}\right), \\
& c=\frac{g_{s}^{2}}{2} \rho^{3}\left(\left|F_{0}\right|^{2}+\left|F_{2}\right|^{2} \rho^{-2}\right) .
\end{aligned}
$$

In our case, asking for $\sigma=1$ and using the extremization in $\sigma$ in (3.93), which can be written as $2 a-3 b+4 c=0$, we find that the minimum in $\sigma_{-}$corresponds to

$$
a-2 c<0 .
$$

\footnotetext{
${ }^{27}$ Since the polynomial is real, they come in conjugate pairs.
} 
This condition is satisfied by our solution choosing $\gamma^{2}=\frac{1}{2}$, as we can see from (3.34). Therefore, our solution is at the minimum in $\sigma$, and it is then stable both in the volume and the dilaton moduli.

It is easy to see that the four-dimensional potential takes a positive value at the minimum, and, hence, the minimum corresponds to a de Sitter vacuum. In [15], it has been shown that the potential has a strictly positive minimum in $\sigma$ for

$$
1<\frac{4 a c}{b^{2}}<\frac{9}{8}
$$

where the lower bound comes from asking the potential to be never vanishing (strictly positive). This condition is satisfied by our solution.

In addition, we can actually compute the value of the potential at $\sigma=\rho=1$. Starting from (3.88) and using the two equations of (3.93), we obtain

$$
\frac{U_{E}}{M_{4}^{2}}=\frac{1}{3}\left(\frac{g_{s}}{2}\left(T_{0}-T\right)+g_{s}^{2}\left|F_{0}\right|^{2}-|H|^{2}\right) .
$$

Using (3.16) and (3.94), one can show that the four-dimensional Ricci scalar is proportional to (3.101), $R_{4}=2 U_{E} / M_{4}^{2}$. For $\gamma^{2}=1 / 2, R_{4}$ is positive (see the discussion below (3.35)), and hence so is the value of the potential at the minimum.

Note also that, for $\gamma^{2}=1 / 2$, the last two terms in (3.101) cancel each other and the entire contribution to the cosmological constant comes from sources, $\left(T_{0}-T\right)$. For supersymmetry breaking branes, this contribution is never vanishing but, for generic situations, we do not know what its sign is. It would be nice to have a model independent argument to determine whether, for this mechanism of supersymmetry breaking, the resulting four-dimensional space is always de Sitter.

As a further check of the existence of a de Sitter minimum for our solution, we can plot in figure 2 the four-dimensional potential $U_{E}$ as a function of $\sigma$ and $\rho$ for some values of the parameters

$$
\begin{aligned}
t_{1}=t_{2}=t_{3}=\tau_{3}=\tau_{6} & =1, \\
q_{1}=1, & q_{2}=3, \quad p=\frac{\cosh ^{-1}(2)}{\pi}, \\
\lambda=5, & \gamma=\frac{1}{\sqrt{2}},
\end{aligned}
$$

\section{Acknowledgments}

We would like to thank Ch. Bock, A. Tomasiello and D. Tsimpis for numerous useful discussions; helpful discussions with D. Cassani, A. Dabholkar, U. Danielsson, M. Goodsell, A. Kashani-Poor, P. Koerber, S. Rollenske, M. Rubin, W. Schulgin, S. Theisen and T. Van Riet are also gratefully acknowledged. R.M. would like to thank Max Planck Institute for gravitational physics at Potsdam for hospitality and A. von Humboldt foundation for support. This work is supported in part by ANR grants BLAN05-0079-01 (DA and MP) and BLAN06-3-137168 (EG and RM). 

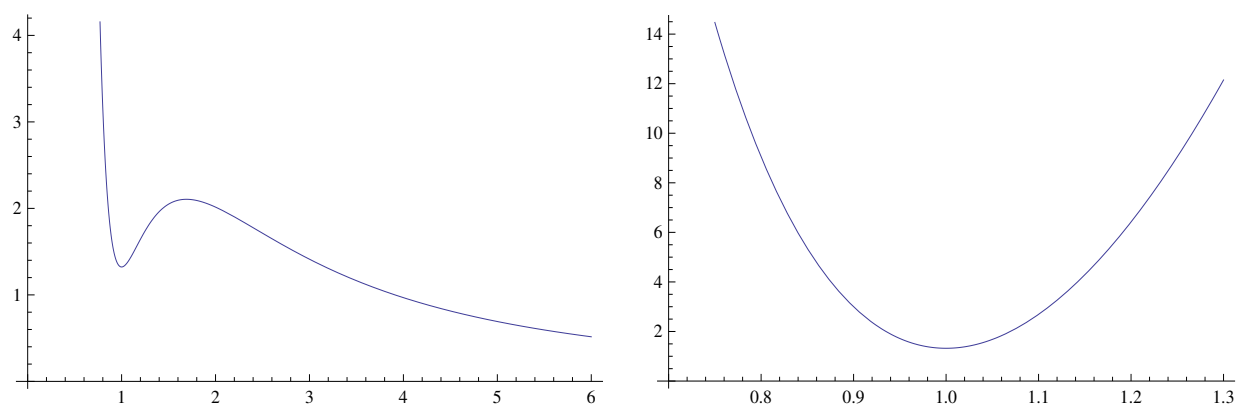

$$
\frac{1}{M_{4}^{2}} U_{E}(\sigma, \rho=1)
$$

$$
\frac{1}{M_{4}^{2}} U_{E}(\sigma=1, \rho)
$$

Figure 2. Dependence of the potential on dilaton and volume modulus.

\section{A Solvable algebras and the geometry of solvmanifolds}

\section{A.1 Algebraic aspects}

We consider a connected and simply-connected real Lie group $G$ of identity element e. $H$, $N$ and $\Gamma$ will be subgroups of $G$. We denote the associated Lie (sub)algebras of $G, H, N$ by $\mathfrak{g}, \mathfrak{h}, \mathfrak{n}$. Connected and simply-connected (sub)groups are in one-to-one correspondence with the corresponding (sub)algebras. Many properties of the (sub)algebras will have their counterpart in the (sub)groups and vice versa.

The ascending series $\left(G_{k}\right)_{k \in \mathbb{N}}$, the descending series $\left(G^{k}\right)_{k \in \mathbb{N}}$ and the derived series $\left(D^{k} G\right)_{k \in \mathbb{N}}$ of subgroups of $G$ are defined as

$$
\begin{aligned}
G_{0} & =\{e\}, & G^{0} & =D^{0} G=G, \\
G_{k} & =\left\{g \in G \mid[g, G] \subset G_{k-1}\right\}, & G^{k} & =\left[G, G^{k-1}\right], D^{k} G=\left[D^{k-1} G, D^{k-1} G\right],
\end{aligned}
$$

where the commutator of two group elements $g$ and $h$ is $[g, h]=g h g^{-1} h^{-1}$. We define in the same way the ascending, descending and derived series of $\mathfrak{g}$ or its subalgebras, by using the Lie bracket instead of the commutator, and 0 instead of $e$.

$G$ is nilpotent respectively solvable if there exist $k$ such that $G^{k}=\{e\}$ respectively $D^{k} G=\{e\}$. We define the same notions for the algebra $\mathfrak{g}$ replacing 0 with $e$. Lie (sub)algebras corresponding to nilpotent/solvable groups are nilpotent and solvable, respectively. The converse is also true. All nilpotent Lie algebras/groups are solvable (the converse is not true).

An ideal $\mathfrak{i}$ of $\mathfrak{g}$ is a subspace of $\mathfrak{g}$ stable under the Lie bracket: $[\mathfrak{g}, \mathfrak{i}] \subset \mathfrak{i}$. Obviously $\mathfrak{i}$ is also a subalgebra. The subalgebras given in the previously defined series are all ideals.

The nilradical $\mathfrak{n}$ of the algebra $\mathfrak{g}$ is the biggest nilpotent ideal of $\mathfrak{g}$. The nilradical is unique $[35,51]$ as will be the corresponding subgroup $N$ of $G$, also named nilradical.

To ideals of $\mathfrak{g}$ will correspond normal subgroups of $G$. We recall that a subgroup $N$ is said normal if $\forall g \in G, g N g^{-1} \subset N$, i.e. it is invariant under conjugation (inner automorphisms). This property is necessary in order to be able to define a group structure 
on the quotient $G / N$. Note that the nilradical $N$ of a solvable Lie group $G$ as well as the subgroups $D^{k} G$ of the derived serie are normal subgroups.

\section{A.1.1 The adjoint action}

Let $V$ be a vector space over a field $\mathbb{K}$ and let $\mathfrak{g}$ be a Lie algebra over the same field. A representation of $\mathfrak{g}$ is a map $\pi: \mathfrak{g} \rightarrow \operatorname{End}(V)$ such that:

1. $\pi$ is linear ;

2. $\pi([X, Y])=\pi(X) \pi(Y)-\pi(Y) \pi(X)$.

There is a natural representation of a Lie algebra over itself called the adjoint representation:

$$
\begin{aligned}
a d: \mathfrak{g} & \rightarrow \operatorname{End}(|\mathfrak{g}|) \\
X & \mapsto a d(X)=a d_{X},
\end{aligned}
$$

where $|\mathfrak{g}|$ means the underlying vector space of the Lie algebra $\mathfrak{g}, \operatorname{End}(|\mathfrak{g}|)$ the space of all linear maps on it, ${ }^{28}$ and

$$
\text { for } \begin{aligned}
X \in \mathfrak{g}, a d_{X}: \mathfrak{g} & \rightarrow \mathfrak{g} \\
Y & \mapsto a d_{X}(Y)=[X, Y] .
\end{aligned}
$$

We can obtain a matrix form of the adjoint representation from the structure constants in a certain basis of the Lie algebra. Let $\left\{E_{a}\right\}_{a=1, \ldots, d}$ be a basis of a Lie algebra $\mathfrak{g}$, and the structure constants in that basis given by

$$
\left[E_{b}, E_{c}\right]=f^{a}{ }_{b c} E_{a} .
$$

Then the matrices ( $a$ is the row index and $c$ is the column index)

$$
\left(M_{b}\right)_{c}^{a}=f_{b c}^{a}
$$

provide a representation of the Lie algebra $\mathfrak{g}$.

A unimodular algebra $\mathfrak{g}$ is such that $\forall X \in \mathfrak{g}, \operatorname{tr}\left(a d_{X}\right)=0$. In view of what has been discussed, this is equivalent to $\sum_{a} f^{a}{ }_{b a}=0, \forall b$.

Let $G$ be a Lie group and let $V$ be a (real) vector space. A representation of $G$ in $V$ is a $\operatorname{map} \pi: G \rightarrow \operatorname{Aut}(V)$ such that:

1. $\pi(e)=I d ;$

2. $\pi\left(g_{1} g_{2}\right)=\pi\left(g_{1}\right) \pi\left(g_{2}\right), \forall g_{1}, g_{2} \in G$.

\footnotetext{
${ }^{28}$ These maps do not necessarily respect the Lie bracket, or in other words, are not necessarily algebra morphisms. In particular, for $X \in \mathfrak{g}, a d_{X}$ is not an algebra morphism.
} 
There is a natural representation of the group over its algebra called the adjoint representation:

$$
\begin{aligned}
A d: G & \rightarrow \operatorname{Aut}(\mathfrak{g}) \\
g & \mapsto \operatorname{Ad}(g)=A d_{g},
\end{aligned}
$$

where $A d_{g}=\exp ^{A u t(|\mathfrak{g}|)}\left(a d_{X_{g}}\right)$ for $X_{g} \in \mathfrak{g}, \exp ^{G}\left(X_{g}\right)=g$. Actually one can show the following relations between the representations:

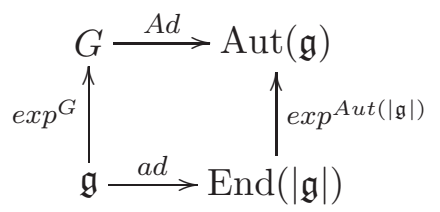

The map ad then turns out to be the derivation ${ }^{29}$ of $A d$. At the level of the single elements, they act according to the following diagram:

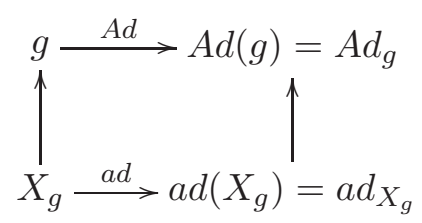

One can show as well that the derivation of the inner automorphism $I_{g}$ for $g \in G$ (the conjugation) is actually the adjoint action $A d_{g}$ :

$$
d\left(I_{g}\right)=A d_{g} .
$$

Furthermore, for $\varphi: G \rightarrow G$ an automorphism, the following diagram is commutative:

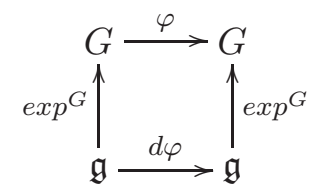

A Lie group is said to be exponential (the case for us) if the exponential map is a diffeomorphism. Denoting its inverse as $\log ^{G}$, then we deduce

$$
I_{g}=\exp ^{G} \circ A d_{g} \circ \log ^{G} .
$$

\section{A.1.2 Semidirect products}

Most of the solvable groups we are interested in are semidirect products, we recall here some definitions.

Let us consider two groups $H$ and $N$ and a (smooth) action $\mu: H \times N \rightarrow N$ by (Lie) automorphisms. The semidirect product of $H$ and $N$ is the group noted $H \ltimes_{\mu} N$, whose underlying set is $H \times N$ and the product is defined as

$$
\left(h_{i=1,2}, n_{i=1,2}\right) \in H \times N,\left(h_{1}, n_{1}\right) \cdot\left(h_{2}, n_{2}\right)=\left(h_{1} \cdot h_{2}, n_{1} \cdot \mu_{h_{1}}\left(n_{2}\right)\right) .
$$

\footnotetext{
${ }^{29}$ It is the derivative with respect to the parameters of the group element $g$, taken at the identity.
} 
The semidirect product of Lie algebras can be defined in a similar way. Let $\mathfrak{d}(\mathfrak{h})$ be the derivation algebra of an algebra $\mathfrak{h}$ (for instance $a d \in \mathfrak{d}(\mathfrak{g})$ ). Let $\sigma: \mathfrak{g} \rightarrow \mathfrak{d}(\mathfrak{h}), X \mapsto \sigma_{X}$ be a representation of the Lie algebra $\mathfrak{g}$ in $|\mathfrak{h}|$. Then we can define the semidirect product $\mathfrak{g} \ltimes_{\sigma} \mathfrak{h}$ of the two Lie algebras with respect to $\sigma$ in the following way:

- the vector space is $|\mathfrak{g}| \times|\mathfrak{h}|$

- the Lie bracket is $\left[\left(X_{1}, Y_{1}\right),\left(X_{2}, Y_{2}\right)\right]=\left(\left[X_{1}, X_{2}\right]_{\mathfrak{g}},\left[Y_{1}, Y_{2}\right]_{\mathfrak{h}}+\sigma_{X_{1}}\left(Y_{2}\right)-\sigma_{X_{2}}\left(Y_{1}\right)\right)$.

This provides a Lie algebra structure to the vector space $|\mathfrak{g}| \times|\mathfrak{h}|$. Note that the fact $\sigma$ is a derivation is important to verify the Jacobi identity for the new bracket.

If we denote $\mathfrak{g}^{\prime}=\mathfrak{g} \times\{0\}$ and $\mathfrak{h}^{\prime}=\{0\} \times \mathfrak{h}$ then $\mathfrak{h}^{\prime}$ is an ideal of the new algebra and $\mathfrak{g}^{\prime}$ is a subalgebra of it. Furthermore

$$
\mathfrak{g}^{\prime}+\mathfrak{h}^{\prime}=\mathfrak{g} \ltimes_{\sigma} \mathfrak{h}, \mathfrak{g}^{\prime} \cap \mathfrak{h}^{\prime}=0
$$

There is a unique decomposition of an element of $|\mathfrak{g}| \times|\mathfrak{h}|$ as a sum of an element of $|\mathfrak{g}|$ and one of $|\mathfrak{h}|$, thus we can think of it as the couple in $|\mathfrak{g}| \times|\mathfrak{h}|$ or as an element of a direct sum of vector spaces.

Let us consider a Lie group $G$ and two subgroups $H$ and $N$ with $N$ normal. If every element of $G$ can be uniquely written as a product of an element in $H$ and one in $N$, then one can show that $G \approx H \ltimes_{\mu} N$ with $\mu$ being the conjugation. ${ }^{30}$ This point of view will be important for us. As discussed previously, the conjugation can be given in terms of the restriction of the adjoint action of $H$ over $\mathfrak{n}$ as in (A.4), so we are able to determine $\mu$ in terms of $A d_{H}(N)$. For exponential groups, as we consider here, the corresponding Lie algebra of $G=H \ltimes_{\mu} N$ is then clearly $\mathfrak{g}=\mathfrak{h} \ltimes_{a d_{\mathfrak{h}}(\mathfrak{n})} \mathfrak{n}$ (we just write $a d$ in the following for simplicity).

Let us now consider a group $G$ with a normal subgroup $N$ of codimension 1 . The Lie algebra $\mathfrak{g}$ has two components, $\mathbb{R}$ and $\mathfrak{n}$. We want to show that $\mathfrak{g}$ is isomorphic to $\mathbb{R} \ltimes_{a d} \mathfrak{n}$, and then, as discussed, we get that $G \approx \mathbb{R} \ltimes_{\mu} N$ with $\mu$ the conjugation. At level of the algebra, in terms of vector spaces, the isomorphism is obviously true. What needs to be verified is that the Lie brackets coincide. The Lie bracket of two elements of $\mathbb{R}$ or of $\mathfrak{n}$ clearly coincide with those of the corresponding two elements of $\mathbb{R} \ltimes_{a d} \mathfrak{n}$. Let us now take $X \in \mathbb{R}, Y \in \mathfrak{n}$. We have for $\mathbb{R} \ltimes_{a d} \mathfrak{n}$ :

$$
[(X, 0),(0, Y)]=\left(0,0+a d_{X}(Y)-a d_{0}(0)\right)=(0,[X, Y])
$$

which clearly coincides with the bracket $[X, Y]$ for $\mathfrak{g}$. We can conclude that $\mathfrak{g}$ is isomorphic to $\mathbb{R} \ltimes_{a d} \mathfrak{n}$ and thus the group is isomorphic to $\mathbb{R} \ltimes_{\mu} N$.

\section{A.1.3 Solvable groups}

According to Levi's decomposition, any real finite dimensional Lie algebra is the semidirect sum of its largest solvable ideal called the radical, and a semi-simple subalgebra. So solvable and nilpotent algebras do not enter the usual Cartan classification. Solvable algebras $\mathfrak{g}$ are

\footnotetext{
${ }^{30}$ In particular it is the case for a group $G=H \ltimes{ }_{\nu} N$ with $\nu$ being not the conjugation.
} 
classified with respect to the dimension of their nilradical $\mathfrak{n}$. One can show $[33,38]$ that $\operatorname{dim} \mathfrak{n} \geq \frac{1}{2} \operatorname{dim} \mathfrak{g}$. Since we are interested in six dimensional manifolds we will consider $\operatorname{dim} \mathfrak{n}=3, \ldots, 6$. If $\operatorname{dim} \mathfrak{n}=6, \mathfrak{n}=\mathfrak{g}$ and the algebra is nilpotent (they clearly are a subset of the solvable ones). There are 34 (isomorphism) classes of six-dimensional nilpotent algebras (see for instance $[41,52]$ for a list), among which 24 are indecomposable. Among the 10 decomposable algebras, there is of course the abelian one, $\mathbb{R}^{6}$. There are 100 indecomposable solvable algebras with $\operatorname{dim} \mathfrak{n}=5$ (99 were found in [53], and [39] added 1, see [36] for a complete and corrected list), and 40 indecomposable solvable algebras with $\operatorname{dim} \mathfrak{n}=4$ [39]. Finally, those with $\operatorname{dim} \mathfrak{n}=3$ are decomposable into sums of two solvable algebras. There are only 2 of them, see Corollary 1 of [54]. In total, there are 164 indecomposable six-dimensional solvable algebras. For a list of six-dimensional indecomposable unimodular ${ }^{31}$ solvable algebras, see [33].

Most of the solvable groups are semidirect products. For $G$ a solvable group and $N$ its nilradical, we consider the following definitions:

- If $G=\mathbb{R} \ltimes_{\mu} N, G$ is called almost nilpotent. All three and four-dimensional solvable groups are of that kind [33].

- If furthermore, the nilradical is abelian (i.e. $N=\mathbb{R}^{k}$ ), $G$ is called almost abelian.

The result at the end of the previous section applies here: any solvable group for which $\operatorname{dim} N=\operatorname{dim} G-1$ is almost nilpotent. In fact $N$ is a normal subgroup of $G$. Let us label the $\mathbb{R}$ direction with a parameter $t$, which we can take as a coordinate, with the corresponding algebra element being $\partial_{t}$. According to (A.4), we then have

$$
\mu(t)=\exp ^{N} \circ A d_{e^{t \partial_{t}}}(\mathfrak{n}) \circ \log ^{N}, A d_{e^{t \partial_{t}}}(\mathfrak{n})=e^{a d_{t \partial_{t}}(\mathfrak{n})}=e^{t a d_{\partial_{t}}(\mathfrak{n})} .
$$

Furthermore, for the almost abelian case, we can identify $N$ and $\mathfrak{n}$, so the exp and $l o g$ correspond to the identity. Then, we obtain the simpler formula

$$
\mu(t)=A d_{e^{t \partial_{t}}}(\mathfrak{n})=e^{t a d_{\partial_{t}}(\mathfrak{n})} .
$$

We will mainly focus on solvable algebras with $\operatorname{dim} \mathfrak{n}=5$ (to which correspond almost nilpotent solvable groups) because, as we will discuss further, the compactness question is simpler to deal with.

\section{A.2 Compactness}

We recall here that according to the definition ${ }^{32}$ we adopt in this paper (section 2) a solvmanifold is a compact homogeneous space $G / \Gamma$ obtained by the quotient of a connected, simply-connected solvable group and a discrete cocompact subgroup $\Gamma$, the lattice [32, 33]. The main result concerning the geometry of these manifolds is the Mostow bundle, and we refer to section 2 for its discussion (see in particular diagram (2.6) and [37] for the original reference). In this appendix, we come back to the problem of the existence of a lattice.

\footnotetext{
${ }^{31}$ See appendix A.1.1 for a definition.

${ }^{32}$ Let us emphasize the non-trivial result that, according to our (restrictive) definition solvmanifolds, these are always parallelizable (see [32] for a proof).
} 
Whetheer a lattice exists or not, and so whether the manifold can be made compact is not always an easy question for non-nilpotent solvable groups. There is a simple necessary condition for a manifold to be compact, namely that the algebra has to be unimodular. Sufficient conditions are on the contrary more difficult to establish.

A theorem by Malcev [30] states that a cornnected and simply-connected nilpotent Lie group $G$ admits a lattice if and only if there exists a basis for the Lie algebra $\mathfrak{g}$ such that the structure constants are rational numbers. This condition is always satisfied for all the 34 classes of nilpotent six dimensional algebras. For the non-nilpotent cases, several criteria have been proposed. The first is due to Auslander [35]. Despite its generality the criterion is difficult to use in concrete situations and we will not refer to it in our search for lattices. Details about it can be found in the original paper [35] and in [33]. Another criterion, which is closer to the one we use in this paper, is due to Saitô [55]. It is less general than Auslander's because it applies to solvable groups that are algebraic subgroups of $G L(n, \mathbb{R})$ for some $n$. The criterion deals with the adjoint action of the group $G$ over the nilradical $\mathfrak{n}$ of its algebra $\mathfrak{g}$. For an illustration, see [41].

The criterion we adopt in this paper follows [33] and it applies to almost abelian solvable groups. As discussed above almost abelian solvable groups are characterized by the map $\mu(t)$ (A.9). Then the criterion states the group $G$ admits a lattice if and only if it exists a $t_{0} \neq 0$ for which $\mu\left(t_{0}\right)$ can be conjugated to an integer matrix. This criterion is very useful in practice since we have a simple formula (A.9) for $\mu(t)$.

In [33], some almost nilpotent (not almost abelian) cases were also proved to admit a lattice, thanks to some further technique that we will not consider here.

In section 2 we applied the compactness criterion mentioned above to the two algebras $\varepsilon_{2}$ and $\varepsilon_{1,1}$ (corresponding to $\mathfrak{g}_{3.5}^{0}$ and $\mathfrak{g}_{3.4}^{-1}$ given in the table 1 , respectively). Here we will review the argument for $\varepsilon_{1,1}$, using a change of basis closer to [33]. The algebra $\varepsilon_{1,1}$ is defined by

$$
\left[E_{1}, E_{3}\right]=E_{1},\left[E_{2}, E_{3}\right]=-E_{2} .
$$

We have $\mathfrak{n}=\left\{E_{1}, E_{2}\right\}$ and $\partial_{t}=E_{3}$. Then, in the $\left(E_{1}, E_{2}\right)$ basis,

$$
a d_{\partial_{t}}(\mathfrak{n})=\left(\begin{array}{cc}
-1 & 0 \\
0 & 1
\end{array}\right), \mu(t)=e^{t a d_{\partial_{t}}(\mathfrak{n})}=\left(\begin{array}{cc}
e^{-t} & 0 \\
0 & e^{t}
\end{array}\right) .
$$

It is not possible to have $\mu\left(t_{0}\right)$ being an integer matrix for $t_{0} \neq 0$. To check if the group admits a lattice, we have to find another basis where the matrix $\mu\left(t_{0}\right)$ can be integer. Let us consider the particular change of basis given by

$$
P=\left(\begin{array}{ll}
1 & c \\
1 & \frac{1}{c}
\end{array}\right), P^{-1}=\frac{1}{c-\frac{1}{c}}\left(\begin{array}{cc}
-\frac{1}{c} & c \\
1 & -1
\end{array}\right),
$$

where $c=e^{-t_{1}}$ and $t_{1} \neq 0$. Then:

$$
\hat{\mu}(t)=P^{-1}\left(\begin{array}{cc}
e^{-t} & 0 \\
0 & e^{t}
\end{array}\right) P=\left(\begin{array}{cc}
\frac{\sinh \left(t_{1}-t\right)}{s_{1}} & -\frac{\sinh (t)}{s_{1}} \\
\frac{\sinh (t)}{s_{1}} & \cosh (t)+c_{1} \frac{\sinh (t)}{s_{1}}
\end{array}\right),
$$




\begin{tabular}{|c|c|c|}
\hline Name & Algebra & \\
\hline $\mathfrak{g}_{3.4}^{-1}$ & {$\left[X_{1}, X_{3}\right]=X_{1},\left[X_{2}, X_{3}\right]=-X_{2}$} & alm. ab. \\
\hline $\mathfrak{g}_{3.5}^{0}$ & {$\left[X_{1}, X_{3}\right]=-X_{2},\left[X_{2}, X_{3}\right]=X_{1}$} & alm. ab. \\
\hline $\mathfrak{g}_{4.5}^{p,-p-1}$ & {$\left[X_{1}, X_{4}\right]=X_{1},\left[X_{2}, X_{4}\right]=p X_{2},\left[X_{3}, X_{4}\right]=-(p+1) X_{3},-\frac{1}{2} \leq p<0$} & alm. ab. \\
\hline $\mathfrak{g}_{4.6}^{-2 p, p}$ & {$\left[X_{1}, X_{4}\right]=-2 p X_{1},\left[X_{2}, X_{4}\right]=p X_{2}-X_{3},\left[X_{3}, X_{4}\right]=X_{2}+p X_{3}, p>0$} & alm. ab. \\
\hline $\mathfrak{g}_{4.8}^{-1}$ & {$\left[X_{2}, X_{3}\right]=X_{1},\left[X_{2}, X_{4}\right]=X_{2},\left[X_{3}, X_{4}\right]=-X_{3}$} & alm. nil. \\
\hline $\mathfrak{g}_{4.9}^{0}$ & {$\left[X_{2}, X_{3}\right]=X_{1},\left[X_{2}, X_{4}\right]=-X_{3},\left[X_{3}, X_{4}\right]=X_{2}$} & alm. nil. \\
\hline
\end{tabular}

Table 1. Indecomposable non-nilpotent solvable unimodular algebras up to dimension 4, that admit a lattice.

with $s_{1}=\sinh \left(t_{1}\right)$ and $c_{1}=\cosh \left(t_{1}\right)$. For $t=t_{1}$, we get

$$
\hat{\mu}\left(t=t_{1}\right)=\left(\begin{array}{cc}
0 & -1 \\
1 & 2 c_{1}
\end{array}\right) .
$$

The conjugated matrix $\hat{\mu}(t)$ can have integers entries for some non-zero $t=t_{1}$ when $2 \cosh \left(t_{1}\right)$ is integer. In [33], $2 \cosh \left(t_{1}\right)=3$.

Let us now describe an example for which there is no lattice. We consider the algebra $\mathfrak{g}_{4.2}^{-p}$

$$
\left[E_{1}, E_{4}\right]=-p E_{1},\left[E_{2}, E_{4}\right]=E_{2},\left[E_{3}, E_{4}\right]=E_{2}+E_{3}, p \neq 0 .
$$

It is easy to check that the algebra is unimodular only for $p=2$. This is a necessary condition for compactness, we can exclude all other values of $p$.

We have $\mathfrak{n}=\left\{E_{1}, E_{2}, E_{3}\right\}$ and $\partial_{t}=E_{4}$ (the algebra is almost abelian). Then, in the $\left(E_{1}, E_{2}, E_{3}\right)$ basis,

$$
a d_{\partial_{t}}(\mathfrak{n})=\left(\begin{array}{ccc}
p & 0 & 0 \\
0 & -1 & 0 \\
0 & -1 & -1
\end{array}\right), \mu(t)=e^{t a d_{\partial_{t}}(\mathfrak{n})}=\left(\begin{array}{ccc}
e^{p t} & 0 & 0 \\
0 & e^{-t} & 0 \\
0 & -t e^{-t} & e^{-t}
\end{array}\right) .
$$

Following [33], we are going to prove that this matrix cannot be conjugated to an integer matrix $^{33}$ except for $t=0$. A way to verify if the matrix $\mu(t)$ can be conjugated to an integer one is to look at the coefficients of its characteristic polynomial $P(\lambda)$. This is independent of the basis in which it is computed, and hence, for the criterion to be satisfied it should have integer coefficients. Here we have:

$$
P(\lambda)=\left(\lambda-e^{2 t}\right)\left(\lambda-e^{-t}\right)^{2}=\lambda^{3}-\lambda^{2}\left(2 e^{-t}+e^{2 t}\right)+\lambda\left(e^{-2 t}+2 e^{t}\right)-1 .
$$

The coefficients are given by sums and products of roots. We can use Lemma (2.2) in [56]. Let

$$
P(\lambda)=\lambda^{3}-k \lambda^{2}+l \lambda-1 \in \mathbb{Z}[\lambda] .
$$

\footnotetext{
${ }^{33} \mathrm{~A}$ naïve reason one could think of would be that it is due to the off-diagonal piece, but as we are going to show, this piece actually does not contribute.
} 


\begin{tabular}{|c|c|}
\hline Name & Algebra \\
\hline $\mathfrak{g}_{5.7}^{p, q, r}$ & {$\left[X_{1}, X_{5}\right]=X_{1},\left[X_{2}, X_{5}\right]=p X_{2},\left[X_{3}, X_{5}\right]=q X_{3},\left[X_{4}, X_{5}\right]=r X_{4}$,} \\
& $-1 \leq r \leq q \leq p \leq 1, p q r \neq 0, p+q+r+1=0$ \\
\hline $\mathfrak{g}_{5.8}^{-1}$ & {$\left[X_{2}, X_{5}\right]=X_{1},\left[X_{3}, X_{5}\right]=X_{3},\left[X_{4}, X_{5}\right]=-X_{4}$} \\
\hline $\mathfrak{g}_{5.13}^{-1-2 q, q, r}$ & {$\left[X_{1}, X_{5}\right]=X_{1},\left[X_{2}, X_{5}\right]=-(1+2 q) X_{2},\left[X_{3}, X_{5}\right]=q X_{3}-r X_{4},\left[X_{4}, X_{5}\right]=r X_{3}+q X_{4}$,} \\
& $-1 \leq q \leq 0, q \neq-\frac{1}{2}, r \neq 0$ \\
\hline $\mathfrak{g}_{5.14}^{0}$ & {$\left[X_{2}, X_{5}\right]=X_{1},\left[X_{3}, X_{5}\right]=-X_{4},\left[X_{4}, X_{5}\right]=X_{3}$} \\
\hline $\mathfrak{g}_{5.15}^{-1}$ & {$\left[X_{1}, X_{5}\right]=X_{1},\left[X_{2}, X_{5}\right]=X_{1}+X_{2},\left[X_{3}, X_{5}\right]=-X_{3},\left[X_{4}, X_{5}\right]=X_{3}-X_{4}$} \\
\hline $\mathfrak{g}_{5.17}^{p,-p, r}$ & {$\left[X_{1}, X_{5}\right]=p X_{1}-X_{2},\left[X_{2}, X_{5}\right]=X_{1}+p X_{2},\left[X_{3}, X_{5}\right]=-p X_{3}-r X_{4},\left[X_{4}, X_{5}\right]=r X_{3}-p X_{4}$,} \\
& $r \neq 0$ \\
\hline $\mathfrak{g}_{5.18}^{0}$ & {$\left[X_{1}, X_{5}\right]=-X_{2},\left[X_{2}, X_{5}\right]=X_{1},\left[X_{3}, X_{5}\right]=X_{1}-X_{4},\left[X_{4}, X_{5}\right]=X_{2}+X_{3}$} \\
\hline
\end{tabular}

Table 2. Indecomposable solvable unimodular almost abelian algebras of dimension 5, that admit a lattice.

\begin{tabular}{|c|c|}
\hline Name & Algebra \\
\hline $\mathfrak{g}_{6.3}^{0,-1}$ & {$\left[X_{2}, X_{6}\right]=X_{1},\left[X_{3}, X_{6}\right]=X_{2},\left[X_{4}, X_{6}\right]=X_{4},\left[X_{5}, X_{6}\right]=-X_{5}$} \\
\hline $\mathfrak{g}_{6.10}^{0,0}$ & {$\left[X_{2}, X_{6}\right]=X_{1},\left[X_{3}, X_{6}\right]=X_{2},\left[X_{4}, X_{6}\right]=-X_{5},\left[X_{5}, X_{6}\right]=X_{4}$} \\
\hline
\end{tabular}

Table 3. Indecomposable solvable unimodular almost abelian algebras of dimension 6 , for which we know a lattice exists.

Then $P(\lambda)$ has a double root $\lambda_{0} \in \mathbb{R}$ if and only if $\lambda_{0}=+1$ or $\lambda_{0}=-1$ for which $P(\lambda)=\lambda^{3}-3 \lambda^{2}+3 \lambda-1$ or $P(\lambda)=\lambda^{3}+\lambda^{2}-\lambda-1$ respectively.

In our case, we find the double root $e^{-t}$. This means the only way to have this polynomial with integer coefficients is to set $t=0$. Then we can conclude there is no lattice.

\section{A.2.1 Algebras admitting a lattice}

We present in tables 1, 2, 3 a list of indecomposable solvable, non-nilpotent unimodular algebras that admit a lattice (at least for certain values of the parameters $p, q, r$, for instance those chosen in table 4). For dimension up to four the algebras are almost nilpotent or almost abelian. For dimension 5 and 6 , only almost abelian algebras have been considered. For the other six-dimensional indecomposable algebras, we do not know if a lattice exists.

\section{A.3 Algorithmic construction of the one-forms of a solvable group}

Let us consider a connected and simply-connected six-dimensional solvable group $G$. As a manifold, its tangent bundle at the identity is given by $T_{e} G \approx \mathfrak{g}$, and has a basis of vectors $E_{a}(a=1 \ldots 6)$ satisfying

$$
\left[E_{b}, E_{c}\right]=f_{b c}^{a} E_{a}
$$


We will focus on the dual basis of one-forms $e^{a}$ on the cotangent bundle $g^{*} \approx T_{e} G^{*}$, which verify the Maurer-Cartan equation

$$
\mathrm{d} e^{a}=-\frac{1}{2} f_{b c}^{a} e^{b} \wedge e^{c}=-\sum_{b<c} f_{b c}^{a} e^{b} \wedge e^{c} .
$$

We want to consider a transformation $A$ relating the one-forms of $\mathbb{R}^{6}$ to those of $G$ :

$$
A\left(\begin{array}{c}
\mathrm{d} x^{1} \\
\vdots \\
\mathrm{d} x^{6}
\end{array}\right)=\left(\begin{array}{c}
e^{1} \\
\vdots \\
e^{6}
\end{array}\right)
$$

Clearly the one-forms in (A.21) must satisfy the corresponding ${ }^{34}$ Maurer-Cartan equation.

The matrix $A$ should reproduce the different fibrations of the solvable group (the bundle structure is manifest in the Maurer-Cartan equations). Given the general form of solvable groups (a nilradical subgroup $N$ and an abelian left over subgroup $G / N=\mathbb{R}^{\operatorname{dim} G-\operatorname{dim} N}$ ), we will consider $A$ to be a product of two pieces:

$$
A=\left(\begin{array}{c|c}
A_{N} & 0 \\
\hline 0 & \mathbb{I}_{6-\operatorname{dim} N}
\end{array}\right)\left(\begin{array}{c|c}
A_{M} & 0 \\
\hline 0 & \mathbb{I}_{6-\operatorname{dim} N}
\end{array}\right),
$$

where we take $A_{M}$ and $A_{N}$ to be $\operatorname{dim} N \times \operatorname{dim} N$ matrices, and we put the abelian directions of $\mathbb{R}^{\operatorname{dim} G-\operatorname{dim} N}$ in the last entries. $A_{M}$ will provide the non-trivial fibration of $N$ over $\mathbb{R}^{\operatorname{dim} G-\operatorname{dim} N}$, the Mostow bundle fibration of the solvmanifold for the compact case, see section 2 . In turn, $A_{N}$ will provide fibrations inside $N$, the fibrations within the nilmanifold piece for the compact case. If the solvable group is nilpotent, then we take $A_{M}$ to be the identity.

To explicitly construct the matrices $A_{M}$ and $A_{N}$ we will now restrict ourselves to $G=N$ (nilpotent) or $G=\mathbb{R} \ltimes_{\mu} N$ (almost nilpotent).

\section{A.3.1 Mostow bundle structure: $A_{M}$}

We focus on the case of an almost nilpotent group. We identify the $\mathbb{R}$ subalgebra with the direction $x^{6}$. Then we take $\partial_{t}=\partial_{6}$ the basis for the $\mathbb{R}$ subalgebra, and the corresponding one-form $\mathrm{d} x^{6}=\mathrm{d} t$. Then we define

$$
A_{M}=A d_{e^{-t \partial_{t}}}(\mathfrak{n})=e^{-t a d_{\partial_{t}}(\mathfrak{n})}
$$

and

$$
e^{i}=\left(A_{M}\right)^{i}{ }_{k} \mathrm{~d} x^{k} .
$$

Let us prove that this action will give forms which do verify the Maurer-Cartan equation. Consider first the simpler case of an almost abelian group, i.e. with $N=\mathbb{R}^{5}$, which has

\footnotetext{
${ }^{34}$ Whether the exterior derivative is defined on these new forms will not be treated (see footnote 5): we will just define it as the exterior derivative of $\mathbb{R}^{6}$ acting on the left-hand side of (A.21).
} 
$A_{N}=\mathbb{I}_{N}$. Then

$$
\begin{aligned}
\mathrm{d} e^{i} & =\mathrm{d}\left(e^{\left.-t a d_{\partial_{t}}\right)^{i}}{ }_{k} \wedge \mathrm{d} x^{k}\right. \\
& =-\mathrm{d} t \wedge\left(a d_{\partial_{t}} e^{-t a d d_{\partial_{t}}}\right)^{i}{ }_{k} \mathrm{~d} x^{k} \\
& =-\mathrm{d} t \wedge\left(a d_{\partial_{t}}\right)^{i}{ }_{j}\left(e^{-t a d_{\partial_{t}}}\right)^{j}{ }_{k} \mathrm{~d} x^{k} \\
& =-\mathrm{d} t \wedge\left(a d_{\partial_{t}}\right)^{i}{ }_{j} e^{j} \\
\mathrm{~d} e^{i} & =-f^{i}{ }_{t j} \mathrm{~d} t \wedge e^{j} .
\end{aligned}
$$

The fact that we used the adjoint action allows to easily verify the Maurer-Cartan equations.

Expression (A.23) for the matrix $A_{M}$ holds also for the more general case of almost nilpotent algebras. In this case the Maurer-Cartan equations have component in direction $\mathrm{d} t$ and also in the directions of the nilradical. The $t$ dependence is always determined by $A_{M}$ and hence it is not modified by the presence of a non-trivial nilradical. The form of the nilradical matrix, $A_{N}$, is given in the subsection below.

\section{A.3.2 Nilmanifold fibration structure: $A_{N}$}

The matrix $A_{N}$ should reproduce the iterated fibration structure of $N$. The iterated structure is related to the descending serie of $\mathfrak{n}$ noted:

$$
\mathfrak{n}^{k=0 \ldots p} \text { with } \mathfrak{n}^{0}=\mathfrak{n}, \mathfrak{n}^{p}=\{0\}
$$

Every $\mathfrak{n}^{k}$ is an ideal of $\mathfrak{g}$, so $\forall k \geq 1, \mathfrak{n}^{k}=\left[\mathfrak{n}, \mathfrak{n}^{k-1}\right] \subset\left[\mathfrak{g}, \mathfrak{n}^{k-1}\right] \subset \mathfrak{n}^{k-1}$. Let us now define another serie:

$$
\text { For } 1 \leq k \leq p, s^{k}=\left\{E \in \mathfrak{n}^{k-1} \text { with } E \notin \mathfrak{n}^{k}\right\} .
$$

Let us prove some property of this serie. Assume that $\exists X \in s^{p} \bigcap s^{q}, p>q$ with $X \neq 0$. Then $X \in \mathfrak{n}^{p-1} \subset \mathfrak{n}^{p-2} \subset \cdots \subset \mathfrak{n}^{q} \subset \mathfrak{n}^{q-1}$. So $X \in \mathfrak{n}^{q-1}$ and $X \in \mathfrak{n}^{q}$, so $X \notin s^{q}$, which is a contradiction. So $s^{p} \bigcap s^{q}=\{0\}$ for $p \neq q$. Furthermore, we always have $s^{p}=\mathfrak{n}^{p-1}$. So $s^{p-1} \bigcup s^{p}=s^{p-1} \bigcup \mathfrak{n}^{p-1}=\mathfrak{n}^{p-2} \bigcup \mathfrak{n}^{p-1}=\mathfrak{n}^{p-2}$. Assume that $s^{k} \bigcup s^{k+1} \bigcup \cdots \cup s^{p-1} \bigcup s^{p}=$ $\mathfrak{n}^{k-1}$. Then $s^{k-1} \bigcup s^{k} \bigcup \cdots \cup s^{p-1} \bigcup s^{p}=s^{k-1} \bigcup \mathfrak{n}^{k-1}=\mathfrak{n}^{k-2} \bigcup \mathfrak{n}^{k-1}=\mathfrak{n}^{k-2}$. So by recurrence, we get that $\bigcup_{k=1 \ldots p} s^{k}=\mathfrak{n}$. In other words, each element of $\mathfrak{n}$ appears in one and only one element of the serie $s^{\{k\}}$.

Let us give an example: consider the five-dimensional solvable algebra $(0,31,-21,23,24)$ (notations of section 2). We have

$$
\begin{aligned}
\mathfrak{g}=\{1,2,3,4,5\}, \mathfrak{n} & =\{2,3,4,5\}, \mathfrak{n}^{1}=\{4,5\}, \mathfrak{n}^{2}=\{5\}, \mathfrak{n}^{3}=\{0\} \\
s^{1} & =\{2,3\}, \quad s^{2}=\{4\}, \quad s^{3}=\{5\} .
\end{aligned}
$$

The descending serie of $\mathfrak{n}$ is known to be related to the fibration structure of the nilpotent group: each element gives a further fibration. Now we understand that the serie $s^{\{k\}}$ gives us what directions are fibered at each step. The correspondence between basis, fibers and series for a general iteration is given in the following diagram (of course it should be 
understood in terms of group elements instead of algebra elements as given here, see [34]):

$$
\begin{aligned}
& \mathcal{F}^{p-1}=s^{p} \quad \hookrightarrow \mathcal{M}^{p-1}=\mathfrak{n} \\
& \downarrow \\
& \mathcal{F}^{p-2}=s^{p-1} \hookrightarrow \mathcal{M}^{p-2}=\mathcal{B}^{p-1} \\
& \downarrow \\
& \text { : } \\
& \mathcal{F}^{2}=s^{3} \quad \hookrightarrow \quad \mathcal{M}^{2}=\mathcal{B}^{3} \\
& \downarrow \\
& \mathcal{F}^{1}=s^{2} \quad \hookrightarrow \quad \mathcal{M}^{1}=\mathcal{B}^{2} \\
& \downarrow \\
& \mathcal{B}^{1}=s^{1}
\end{aligned}
$$

We see the unique decomposition of $\mathfrak{n}$ into the serie $s^{\{k\}}$. We have $\mathcal{B}^{i}=\bigcup_{k=1 \ldots i} s^{k}$ and $\mathcal{F}^{i}=s^{i+1}$.

The matrix giving a single fibration was worked out in [28], we recover this result here. In the general case of an iteration, we consider a product of several operators, each of them giving one fibration of the iteration:

$$
A_{N}=A_{p-1} \ldots A_{1}, A_{i}=e^{-\frac{1}{2} f_{i}} \quad\left(\text { for } p=1, \mathfrak{n}=\mathbb{R}^{5} \text { and } A_{N}=1\right),
$$

with $f_{i} \in \operatorname{End}(\mathfrak{n})$ :

$$
\begin{aligned}
& \text { For } i=1 \ldots p-1, f_{i}: \mathfrak{n} \rightarrow \mathfrak{n} \\
& \qquad \begin{aligned}
X \mapsto Y=a d_{\mathcal{B}^{i}}(X) \text { if } X \in \mathcal{B}^{i} \text { and } a d_{\mathcal{B}^{i}}(X) \in \mathcal{F}^{i}, \\
Y=0 \text { otherwise } .
\end{aligned}
\end{aligned}
$$

We choose to give a basis of $\mathfrak{n}$ in the order given by $s^{1}, s^{2}, \ldots, s^{p}$, and in each $s^{k}$ we can choose some order for the elements. Then in that basis, $f_{i}$, as a matrix, is an off-diagonal block with lines corresponding to $\mathcal{F}^{i}=s^{i+1}$ and columns to $\mathcal{B}^{i}=\bigcup_{k=1 \ldots i} s^{k}$. Then $A_{i}$ is the same plus the identity. Furthermore, the block depends on parameters $a^{j}$ of a generic element $a^{j} E_{j}$ of $\mathcal{B}^{i}$, and we have $a d_{a^{j} E_{j} \in \mathcal{B}^{i}}=a^{j} a d_{E_{j} \in \mathcal{B}^{i}}$. So for instance for the previous algebra, we get:

$$
A_{1}=\left(\begin{array}{cccc}
1 & 0 & 0 & 0 \\
0 & 1 & 0 & 0 \\
\frac{1}{2} a^{3} & -\frac{1}{2} a^{2} & 1 & 0 \\
0 & 0 & 0 & 1
\end{array}\right) \quad A_{2}=\left(\begin{array}{cccc}
1 & 0 & 0 & 0 \\
0 & 1 & 0 & 0 \\
0 & 0 & 1 & 0 \\
\frac{1}{2} a^{4} & 0 & -\frac{1}{2} a^{2} & 1
\end{array}\right)
$$

The parameters $a^{j}$ can be understood as a coordinate along $E_{j}$, so they are such that $\mathrm{d} a^{j}=e^{j}$, dual of $E_{j}$.

Let us prove that the operator $A_{r}$ gives the fibration of directions of $\mathcal{F}^{r}$ over a base $\mathcal{B}^{r}$, and the correct corresponding Maurer-Cartan equation. As explained, an element of 
$A_{r}$ is given by:

$$
\left(A_{r}\right)_{k}^{i}=\delta_{k}^{i}-\frac{1}{2} \sum_{j \in \mathcal{B}^{r}} a^{j}\left(a d_{E_{j}}\right)_{k}^{i} \Theta\left(i \in \mathcal{F}^{r}\right) \Theta\left(k \in \mathcal{B}^{r}\right)=\delta_{k}^{i}-\frac{1}{2} \sum_{j, k \in \mathcal{B}^{r}} a^{j} f^{i}{ }_{j k} \Theta\left(i \in \mathcal{F}^{r}\right) .
$$

The forms on which we act with $A_{r}$ at the step $r$ of the iteration are labelled $e^{k}$, and they become after the operation $\tilde{e}^{i}$ :

$$
\tilde{e}^{i}=\left(A_{r}\right)_{k}^{i} e^{k} .
$$

The directions we fiber with $A_{r}$ are initially not fibered, so $e^{k \in \mathcal{F}^{r}}=\mathrm{d} x^{k}$. All the other directions are not modified by $A_{r}$, so in particular $\tilde{e}^{i \in \mathcal{B}^{r}}=e^{i \in \mathcal{B}^{r}}$. So the Maurer-Cartan equations of the forms not in $\mathcal{F}^{r}$ are not modified at this step. Their equation is then only modified at the step when they are fibered, so we don't have to consider it here. For the directions $\mathcal{F}^{r}$, we get:

$$
\begin{aligned}
\tilde{e}^{i \in \mathcal{F}^{r}} & =e^{i \in \mathcal{F}^{r}}-\frac{1}{2} \sum_{j, k \in \mathcal{B}^{r}} a^{j} f^{i}{ }_{j k} e^{k} \\
& =\mathrm{d} x^{i}-\frac{1}{2} \sum_{j, k} a^{j} f^{i}{ }_{j k} e^{k}
\end{aligned}
$$

where we dropped the restriction $j, k \in \mathcal{B}^{r}$ because due to the iterated structure, for $i \in \mathcal{F}^{r}$, $f^{i}{ }_{j k}=0$ if $k$ or $j \notin \mathcal{B}^{r}$. This operation then gives the fibration structure, since we can read the connection. We can verify that we have the correct Maurer-Cartan equation:

$$
\begin{aligned}
\mathrm{d} \tilde{e}^{i \in \mathcal{F}^{r}} & =-\frac{1}{2} f^{i}{ }_{j k} \mathrm{~d} a^{j} \wedge e^{k} \\
& =-\frac{1}{2} f^{i}{ }_{j k} e^{j} \wedge e^{k} \\
\mathrm{~d} \tilde{e}^{i} & =-\frac{1}{2} f^{i}{ }_{j k} \tilde{e}^{j} \wedge \tilde{e}^{k} .
\end{aligned}
$$

\section{A.4 Six-dimensional solvmanifolds in terms of globally defined one-forms}

In table 4 we present the solvmanifolds that we considered in this paper. They have the form $G / \Gamma=H_{1} / \Gamma_{1} \times H_{2} / \Gamma_{2}$, i.e. they are products of (at most) two solvmanifolds. Each of these two solvmanifolds are constructed from the algebras given in the tables 1, 2, 3 (see appendix A.2.1) and the three-dimensional nilpotent algebra $\mathfrak{g}_{3.1}:(-23,0,0)$. In particular, these are indecomposable solvable algebras for which the group admits a lattice. The difference with respect to section A.2.1 is that the algebras are given here in terms of a basis of globally defined forms (see discussion in section 2.1). They are related by isomorphisms to the algebras given in the tables $1,2,3$. The fact the forms are globally defined is important for studying the compatibility of orientifold planes with the manifold and for finding solutions. For $\mathfrak{g}_{4.5}^{p,-p-1} \oplus \mathbb{R}^{2}$ and $\mathfrak{g}_{4.6}^{-2 p, p} \oplus \mathbb{R}^{2}$, we were not able to find such a basis, even if a priori we expect it to exist.

The column Name indicates the label of the algebra and the corresponding solvmanifold. The column Algebra gives the corresponding six-dimensional algebra in terms of exterior derivative acting on the dual basis of globally defined one-forms (see section 2). 
The next two columns give the $\mathrm{O} 5$ and $\mathrm{O} 6$ planes that are compatible with the manifold. The column $S p$ indicates by a $\checkmark$ when the manifold is symplectic, according to [33, 36]. Notice that the same results can be obtained as conditions for the twisted pure spinors to solve the supersymmetry equations. In particular, for the even $\mathrm{SU}(3)$ pure spinor $\Phi_{+}=\frac{1}{8} e^{-i J}$ the condition

$$
\mathrm{d}\left(O_{t w}\right) \Phi_{+}=0
$$

is equivalent to the requirement that the manifold is symplectic.

There is an additional subtlety for not completely solvable manifolds, when one looks for solutions on them. This is due to the lack of isomorphism between the cohomology groups $H^{*}(\mathfrak{g})$ and $H_{d R}^{*}(G / \Gamma)$ for not completely solvable manifolds (see footnote 5 ). In other words, the Betti numbers for the Lie algebra cohomology give only the lower bound for the corresponding numbers for de Rham cohomology. When looking for e.g. symplectic manifolds, we have considered only the forms in $H^{2}(\mathfrak{g})$, and hence might have missed some candidate two-forms in $H_{d R}^{2}(G / \Gamma)$.

\section{B T-dualising solvmanifolds}

T-duality has been extensively used in flux compactifications in order to obtain solutions on nilmanifolds. Being iterations of torus bundles, these are obtainable from torus solutions with an appropriate $B$-field (the contraction of $H$ with the isometry vectors should be a closed horizontal two-form that can be thought as a curvature of the dual torus bundle.). Correspondingly, the structure constants $f^{a}{ }_{b c}$ have also a T-duality friendly form. For any upper index there is a well-defined isometry vector $\partial_{a}$ with respect to which one can perform an (un-obstructed) T-duality.

In this section we would like to study some aspects of T-duality for solvmanifolds. In this case, the situation is more complicated. For instance, it can happen that the structure constants have the same index in the upper and lower position $f_{a c}^{a}$ and are not fully antisymmetric. Put differently, most of our knowledge about the global aspects of Tduality comes form the study of its action on (iterations of) principal U(1) bundles. Since the Mostow bundles are not in general principal, the topology of the T-dual backgrounds is largely unexplored. We shall not attempt to do this here, but rather illustrate some of novel features by considering T-duality on the simplest cases of almost abelian manifolds.

Requiring that T-duality preserves supersymmetry imposes that the Lie derivatives with respect to any isometry vector $v$ vanish, $\mathcal{L}_{v} \Psi_{ \pm}=0$ [43]. For the simple case of almost abelian solvmanifolds, it is not hard to check that all vectors $v_{i}=\partial_{i}$, where, in the basis chosen in this paper, $i=1, \ldots, 4,6$, satisfy this condition. However, these vectors are defined only locally, ${ }^{35}$ since they transform non-trivially under $t \sim t+t_{0}$. Hence, in general, the result of T-duality will be non-geometric. We shall see that there are subtleties even for the case when the supersymmetry-preserving isometries $\partial_{i}$ are well defined.

\footnotetext{
${ }^{35}$ As discussed, on the compact solvmanifolds there exists a set of globally defined one forms $\{e\}=$ $\left\{A_{M} \mathrm{~d} x\right\}$ and the dual basis $\{E\}=\left\{\left(A_{M}^{-1}\right)^{T} \partial\right\}$ is made of globally defined vectors. However, the Lie derivative of the pure spinors with respect to these does not vanish.
} 


\begin{tabular}{|c|c|c|c|c|}
\hline Name & Algebra & O5 & O6 & $\mathrm{Sp}$ \\
\hline $\mathfrak{g}_{3.4}^{-1} \oplus \mathbb{R}^{3}$ & $\left(q_{1} 23, q_{2} 13,0,0,0,0\right) \quad q_{1}, q_{2}>0$ & $\begin{array}{c}14,15,16,24,25 \\
26,34,35,36\end{array}$ & $\begin{array}{l}123,145,146,156,245 \\
246,256,345,346,356\end{array}$ & $\checkmark$ \\
\hline $\mathfrak{g}_{3.5}^{0} \oplus \mathbb{R}^{3}$ & $(-23,13,0,0,0,0)$ & $\begin{array}{c}14,15,16,24,25 \\
26,34,35,36\end{array}$ & $\begin{array}{l}123,145,146,156,245 \\
246,256,345,346,356\end{array}$ & $\checkmark$ \\
\hline $\mathfrak{g}_{3.1} \oplus \mathfrak{g}_{3.4}^{-1}$ & $\left(-23,0,0, q_{1} 56, q_{2} 46,0\right) \quad q_{1}, q_{2}>0$ & $\begin{array}{c}14,15,16,24,25 \\
26,34,35,36\end{array}$ & - & $\checkmark$ \\
\hline $\mathfrak{g}_{3.1} \oplus \mathfrak{g}_{3.5}^{0}$ & $(-23,0,0,-56,46,0)$ & $\begin{array}{c}14,15,16,24,25 \\
26,34,35,36\end{array}$ & - & $\checkmark$ \\
\hline $\mathfrak{g}_{3.4}^{-1} \oplus \mathfrak{g}_{3.5}^{0}$ & $\left(q_{1} 23, q_{2} 13,0,-56,46,0\right) \quad q_{1}, q_{2}>0$ & $\begin{array}{c}14,15,16,24,25 \\
26,34,35,36\end{array}$ & - & $\checkmark$ \\
\hline $\mathfrak{g}_{3.4}^{-1} \oplus \mathfrak{g}_{3.4}^{-1}$ & $\left(q_{1} 23, q_{2} 13,0, q_{3} 56, q_{4} 46,0\right) \quad q_{1}, q_{2}, q_{3}, q_{4}>0$ & $\begin{array}{c}14,15,16,24,25 \\
26,34,35,36\end{array}$ & - & $\checkmark$ \\
\hline $\mathfrak{g}_{3.5}^{0} \oplus \mathfrak{g}_{3.5}^{0}$ & $(-23,13,0,-56,46,0)$ & $\begin{array}{c}14,15,16,24,25 \\
26,34,35,36\end{array}$ & - & $\checkmark$ \\
\hline $\mathfrak{g}_{4.5}^{p,-p-1} \oplus \mathbb{R}^{2}$ & $?$ & & & - \\
\hline $\mathfrak{g}_{4.6}^{-2 p, p} \oplus \mathbb{R}^{2}$ & $?$ & & & - \\
\hline $\mathfrak{g}_{4.8}^{-1} \oplus \mathbb{R}^{2}$ & $\left(-23, q_{1} 34, q_{2} 24,0,0,0\right) \quad q_{1}, q_{2}>0$ & $14,25,26,35,36$ & $145,146,256,356$ & - \\
\hline $\mathfrak{g}_{4.9}^{0} \oplus \mathbb{R}^{2}$ & $(-23,-34,24,0,0,0)$ & $14,25,26,35,36$ & $145,146,256,356$ & - \\
\hline $\mathfrak{g}_{5.7}^{1,-1,-1} \oplus \mathbb{R}$ & $\left(q_{1} 25, q_{2} 15, q_{2} 45, q_{1} 35,0,0\right) \quad q_{1}, q_{2}>0$ & $13,14,23,24,56$ & $125,136,146,236,246,345$ & $\checkmark$ \\
\hline $\mathfrak{g}_{5.8}^{-1} \oplus \mathbb{R}$ & $\left(25,0, q_{1} 45, q_{2} 35,0,0\right) \quad q_{1}, q_{2}>0$ & $13,14,23,24,56$ & $125,136,146,236,246,345$ & $\checkmark$ \\
\hline $\mathfrak{g}_{5.13}^{-1,0, r} \oplus \mathbb{R}$ & $\left(q_{1} 25, q_{2} 15,-q_{2} r 45, q_{1} r 35,0,0\right) r \neq 0, q_{1}, q_{2}>0$ & $13,14,23,24,56$ & $125,136,146,236,246,345$ & $\checkmark$ \\
\hline $\mathfrak{g}_{5.14}^{0} \oplus \mathbb{R}$ & $(-25,0,-45,35,0,0)$ & $13,14,23,24,56$ & $125,136,146,236,246,345$ & $\checkmark$ \\
\hline $\mathfrak{g}_{5.15}^{-1} \oplus \mathbb{R}$ & $\left(q_{1}(25-35), q_{2}(15-45), q_{2} 45, q_{1} 35,0,0\right) \quad q_{1}, q_{2}>0$ & $14,23,56$ & 146,236 & $\checkmark$ \\
\hline $\mathfrak{g}_{5.17}^{p,-p, r} \oplus \mathbb{R}$ & $\begin{array}{c}\left(q_{1}(p 25+35), q_{2}(p 15+45), q_{2}(p 45-15), q_{1}(p 35-25), 0,0\right) \\
r^{2}=1, q_{1}, q_{2}>0\end{array}$ & $\begin{array}{c}14,23,56 \\
p=0: 12,34\end{array}$ & $\begin{array}{c}146,236 \\
p=0: 126,135,245,346\end{array}$ & $\checkmark$ \\
\hline $\mathfrak{g}_{5.18}^{0} \oplus \mathbb{R}$ & $(-25-35,15-45,-45,35,0,0)$ & $14,23,56$ & 146,236 & $\checkmark$ \\
\hline $\mathfrak{g}_{6.3}^{0,-1}$ & $\left(-26,-36,0, q_{1} 56, q_{2} 46,0\right) \quad q_{1}, q_{2}>0$ & 24,25 & $134,135,456$ & $\checkmark$ \\
\hline $\mathfrak{g}_{6.10}^{0,0}$ & $(-26,-36,0,-56,46,0)$ & 24,25 & $134,135,456$ & $\checkmark$ \\
\hline
\end{tabular}

Table 4. Six-dimensional solvmanifolds considered in this paper, in terms of globally defined one-forms. 
We shall consider the action of T-duality on two solvmanifolds, $\mathfrak{g}_{5.17}^{0,0, \pm 1} \times S^{1}(s 2.5)$ and $\mathfrak{g}_{5.7}^{1,-1,-1} \times S^{1}$. For $s 2.5$, following [41], we write the algebra as $(25,-15, r 45,-r 35,0,0)$, $r^{2}=1$. The twist matrix $A(t)$ is made of periodic functions of $t=x^{5}$,

$$
A=\left(\begin{array}{ccc}
R_{r=1} & & \\
& R_{r} & \\
& & \mathbb{I}_{2}
\end{array}\right), \quad R_{r}=\left(\begin{array}{cc}
\cos x^{5} & -r \sin x^{5} \\
r \sin x^{5} & \cos x^{5}
\end{array}\right),
$$

and T-duality is un-obstructed. The various supersymmetric solutions found in [41, 42] are all related by two T-dualities

IIB

$$
\mathrm{t}: 30
$$$$
\mathrm{t}: 12
$$

$$
\begin{aligned}
& (13+24) \stackrel{\mathrm{T}_{12}}{\longleftrightarrow}(14+23) \\
& (14+23) \stackrel{2}{\longleftrightarrow}(13+24)
\end{aligned}
$$

In the table we labelled each solution by the dominant O-plane charge. The sources are labelled by their longitudinal directions, e.g. $(13+24)$ stands for a solution with two sources (one $\mathrm{O} 5$ and one D5) along directions $e^{1} \wedge e^{3}$ and $e^{2} \wedge e^{4}$. T-dualities (the subscripts indicate the directions in which they are performed) exchange the columns in the table; lines are exchanged by relabellings (symmetries of the algebra).

The T-dualities are type ${ }^{36}$ changing, meaning a pair of type 0 and 3 (t:30) pure spinors is exchanged with a pair of type 1 and 2 (t:12) and vice versa.

It is natural to see what will it be the effect of a single T-duality. To be precise we take as starting point Model 3 of [41]. We shall concentrate on the NS sector and discuss the topology changes under T-duality. The NS flux is zero and the metric, in the $\mathrm{d} x^{i}$ basis is

$$
\begin{aligned}
\mathrm{d} s^{2}= & \frac{t_{1}^{2}}{t_{2}}\left(\tau_{2}^{1}\right)^{2} G\left(\mathrm{~d} x^{1}+\mathcal{A} \mathrm{d} x^{2}\right)^{2}+\frac{t_{1}}{G}\left(\mathrm{~d} x^{2}\right)^{2}+t_{1}\left(\tau_{2}^{1}\right)^{2} G\left(\mathrm{~d} x^{3}+r \mathcal{A} \mathrm{d} x^{4}\right)^{2} \\
& +\frac{t_{2}}{G}\left(\mathrm{~d} x^{4}\right)^{2}+t_{3}\left(\mathrm{~d} x^{5}\right)^{2}+t_{3}\left(\mathrm{~d} x^{6}\right)^{2}
\end{aligned}
$$

with

$$
G=\cos ^{2}\left(x^{5}\right)+\frac{t_{2}}{t_{1}\left(\tau_{2}^{1}\right)^{2}} \sin ^{2}\left(x^{5}\right) \quad \mathcal{A}=\frac{t_{2}-t_{1}\left(\tau_{2}^{1}\right)^{2}}{2 G t_{1}\left(\tau_{2}^{1}\right)^{2}} \sin \left(2 x^{5}\right) .
$$

A single T-duality along $x^{1}$ yields the manifold $T^{3} \times \varepsilon_{2}\left(\varepsilon_{2}:(-23,13,0)\right)$ with O7-D5 (or D7-O5) and an $H$-flux given by

$$
H=-\mathrm{d} \mathcal{A} \wedge \mathrm{d} x^{1} \wedge \mathrm{d} x^{2} .
$$

${ }^{36} \mathrm{~A}$ pure spinor $\Psi$ can always be written as

$$
\Psi=e^{B+i j} \Omega_{k},
$$

where $\Omega_{k}$ is a holomorphic $k$-form, $B$ and $j$ are real two-forms. The degree of $\Omega_{k}$ is the type of the pure spinor. 
Note that the $H$-flux (B.5) allows for topologically different choices of $B$-field. Being not completely solvable (see footnote 5), $s 2.5$ can yield manifolds of different topology (different Betti numbers). Correspondingly, the results of T-duality should vary as well, and the application of the local Buscher rules might be ambiguous. The choice of $B$-field in (B.5), $B=-\mathcal{A} \mathrm{d} x^{1} \wedge \mathrm{d} x^{2}$, corresponding to the application of the local rules to (B.3), is globally defined due to $\mathcal{A}\left(x^{5}+l\right)=\mathcal{A}\left(x^{5}\right)$. There is a less trivial choice with $B=-x^{1} \partial_{5} \mathcal{A} \mathrm{d} x^{2} \wedge \mathrm{d} x^{5}$ which however does not arise from the application of local T-duality rules to (B.3) since the metric does not have off-diagonal elements between $x^{2}$ and $x^{5}$.

A further T-duality along $x^{2}$ gives back $s 2.5$ with O6-D6 sources, but the supersymmetry now is captured by a different pair of pure spinors.

For the manifold $\mathfrak{g}_{5.7}^{1,-1,-1} \oplus \mathbb{R}$, the twist matrix is

$$
A\left(x^{5}\right)=\left(\begin{array}{ccc}
R\left(x^{5}\right) & & \\
& R\left(-x^{5}\right) & \\
& & \mathbb{I}_{2}
\end{array}\right) \quad R\left(x^{5}\right)=\left(\begin{array}{cc}
\mathrm{ch} & -\eta \mathrm{sh} \\
-\frac{1}{\eta} \operatorname{sh} & \mathrm{ch}
\end{array}\right),
$$

where we set

$$
\operatorname{ch}=\cosh \left(\sqrt{q_{1} q_{2}} x^{5}\right), \operatorname{sh}=\sinh \left(\sqrt{q_{1} q_{2}} x^{5}\right), \eta=\sqrt{\frac{q_{1}}{q_{2}}} .
$$

Then it is straightforward to check that the isometry vectors $v_{i}=\partial_{i}$ are local. Any Tduality along these is thus obstructed, and hence the O6-D6 solution of [40, 41] does not have geometric T-duals. For this case we shall adopt the method applied to nilmanifolds in [43], and work out the action of T-duality on the generalized vielbeine.

The generalized vielbeine on $\mathfrak{g}_{5.7}^{1,-1,-1} \oplus \mathbb{R}$ can be obtained using twist transformation (see (2.48)) from the generalized vielbeine of the torus (on which we take for simplicity the identity metric)

$$
\mathcal{E}=\left(\begin{array}{c|c}
\mathbb{I}_{6} & 0_{6} \\
\hline 0_{6} & \mathbb{I}_{6}
\end{array}\right)\left(\begin{array}{c|c}
A & 0_{6} \\
\hline 0_{6} & A^{-T}
\end{array}\right)
$$

To work out their T-duals, we act by

$$
\mathcal{E}_{T}=O_{T} \times \mathcal{E} \times O_{T}
$$

where $O_{T}$ is the $\mathrm{O}(d, d)$ matrix for T-duality. The $O_{T}$ on the right is the regular action of T-duality, while the $O_{T}$ on the left assures that the map has no kernel (see [43]). The T-duality is done in the $x^{1}$ direction, so the $O_{T}$ is

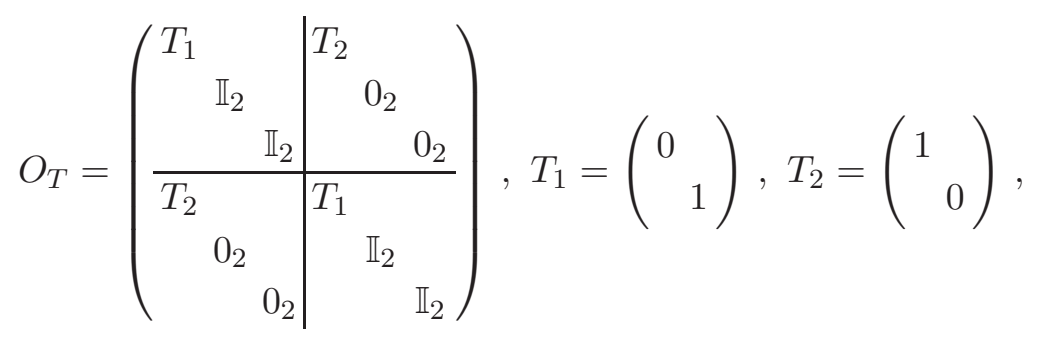


and then

$$
\mathcal{E}_{T}=\left(\begin{array}{ccc|ccc}
C_{1} & & & B_{1} & & \\
& R\left(-x^{5}\right) & & 0_{2} & \\
& & \mathbb{I}_{2} & & & 0_{2} \\
\hline B_{2} & & & C_{2} & \\
& 0_{2} & & & R\left(x^{5}\right)^{T} & \\
& & 0_{2} & & & \mathbb{I}_{2}
\end{array}\right),
$$

with

$$
C_{1}=C_{2}=\operatorname{ch} \mathbb{I}_{2}, B_{1}=-\frac{1}{\eta} \operatorname{sh} \epsilon, B_{2}=\eta \operatorname{sh} \epsilon, \epsilon=\left(\begin{array}{cc}
0 & -1 \\
1 & 0
\end{array}\right) .
$$

The generalized vielbeine $\mathcal{E}_{T}$ can be brought to the canonical lower diagonal form (2.46) by a local $O(d) \times O(d)$ transformation. When such a transformation cannot be made single-valued, we talk about non-geometric backgrounds (where the action of a non-trivial $\beta$ cannot be gauged away). The result of the $O(d) \times O(d)$ transformation is

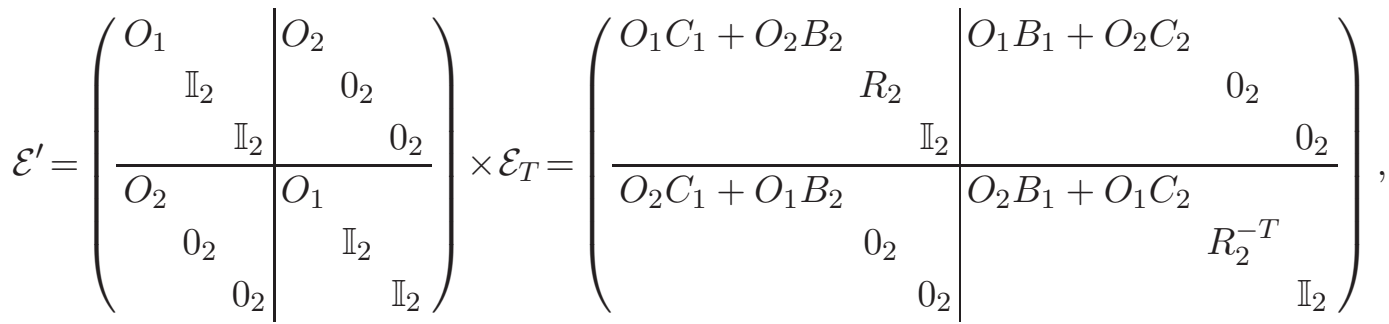

where the non-trivial $O(d) \times O(d)$ components are

$$
O_{1 / 2}=\frac{1}{2}\left(O_{+} \pm O_{-}\right) \quad O_{ \pm} \in O(2) .
$$

By solving $O_{1} B_{1}+O_{2} C_{2}=0$, we can obtain $O_{2}$ and express $O_{ \pm}$in terms of $O_{1}$ :

$$
\begin{aligned}
O_{ \pm} & =O_{1}\left(\mathbb{I}_{2} \pm u \epsilon\right), \quad u=\frac{\mathrm{sh}}{\eta \mathrm{ch}}, \\
O_{ \pm}^{T} O_{ \pm} & =\mathbb{I}_{2} \quad \Leftrightarrow \quad O_{1}^{T} O_{1}=\frac{1}{1+u^{2}} \mathbb{I}_{2} .
\end{aligned}
$$

A simple solution is given by

$$
O_{1}=\frac{1}{\sqrt{1+u^{2}}} \mathbb{I}_{2} \quad \Rightarrow \quad O_{2}=\frac{u}{\sqrt{1+u^{2}}} \epsilon .
$$

Thus we can indeed bring $\mathcal{E}_{T}$ to a lower-diagonal form, but with an $O(d) \times O(d)$ transformation that is not globally defined. It is not hard to see that replacing the $x^{1}$ direction by others does not change much. Hence any T-dual to $\mathfrak{g}_{5.7}^{1,-1,-1} \times S^{1}$ is non-geometric.

A similar analysis for $s 2.5$ shows that one can easily solve the constraint $O_{1} B_{1}+O_{2} C_{2}=$ 0 with $O_{1}$ and $O_{2}$ being globally defined (this is easy since the functions entering are all periodic).

Open Access. This article is distributed under the terms of the Creative Commons Attribution Noncommercial License which permits any noncommercial use, distribution, and reproduction in any medium, provided the original author(s) and source are credited. 


\section{References}

[1] M. Graña, Flux compactifications in string theory: A comprehensive review, Phys. Rept. 423 (2006) 91 [hep-th/0509003] [SPIRES].

[2] M.R. Douglas and S. Kachru, Flux compactification, Rev. Mod. Phys. 79 (2007) 733 [hep-th/0610102] [SPIRES].

[3] R. Blumenhagen, B. Körs, D. Lüst and S. Stieberger, Four-dimensional String Compactifications with D-branes, Orientifolds and Fluxes, Phys. Rept. 445 (2007) 1 [hep-th/0610327] [SPIRES].

[4] D. Lüst and D. Tsimpis, Supersymmetric AdS $S_{4}$ compactifications of IIA supergravity, JHEP 02 (2005) 027 [hep-th/0412250] [SPIRES].

[5] J.P. Gauntlett, D. Martelli, J. Sparks and D. Waldram, Supersymmetric AdS $S_{5}$ solutions of type IIB supergravity, Class. Quant. Grav. 23 (2006) 4693 [hep-th/0510125] [SPIRES].

[6] P. Koerber and D. Tsimpis, Supersymmetric sources, integrability and generalized-structure compactifications, JHEP 08 (2007) 082 [arXiv:0706.1244] [SPIRES].

[7] N. Hitchin, Generalized Calabi-Yau manifolds, Quart. J. Math. Oxford Ser. 54 (2003) 281 [math/0209099].

[8] M. Gualtieri, Generalized complex geometry, math/0401221.

[9] M. Graña, R. Minasian, M. Petrini and A. Tomasiello, Supersymmetric backgrounds from generalized Calabi-Yau manifolds, JHEP 08 (2004) 046 [hep-th/0406137] [SPIRES].

[10] M. Graña, R. Minasian, M. Petrini and A. Tomasiello, Generalized structures of $\mathcal{N}=1$ vacua, JHEP 11 (2005) 020 [hep-th/0505212] [SPIRES].

[11] D. Lüst, F. Marchesano, L. Martucci and D. Tsimpis, Generalized non-supersymmetric flux vacua, JHEP 11 (2008) 021 [arXiv:0807.4540] [SPIRES].

[12] J.M. Maldacena and C. Núñez, Supergravity description of field theories on curved manifolds and a no go theorem, Int. J. Mod. Phys. A 16 (2001) 822 [hep-th/0007018] [SPIRES].

[13] M. Ihl, D. Robbins and T. Wrase, Toroidal Orientifolds in IIA with General NS-NS Fluxes, JHEP 08 (2007) 043 [arXiv:0705.3410] [SPIRES].

[14] M.P. Hertzberg, S. Kachru, W. Taylor and M. Tegmark, Inflationary Constraints on Type IIA String Theory, JHEP 12 (2007) 095 [arXiv:0711.2512] [SPIRES].

[15] E. Silverstein, Simple de Sitter Solutions, Phys. Rev. D 77 (2008) 106006 [arXiv:0712.1196] [SPIRES].

[16] S.S. Haque, G. Shiu, B. Underwood and T. Van Riet, Minimal simple de Sitter solutions, Phys. Rev. D 79 (2009) 086005 [arXiv:0810.5328] [SPIRES].

[17] U.H. Danielsson, S.S. Haque, G. Shiu and T. Van Riet, Towards Classical de Sitter Solutions in String Theory, JHEP 09 (2009) 114 [arXiv:0907.2041] [SPIRES].

[18] C. Caviezel et al., On the Cosmology of Type IIA Compactifications on SU(3)-structure Manifolds, JHEP 04 (2009) 010 [arXiv:0812.3551] [SPIRES].

[19] R. Flauger, S. Paban, D. Robbins and T. Wrase, On Slow-roll Moduli Inflation in Massive IIA Supergravity with Metric Fluxes, Phys. Rev. D 79 (2009) 086011 [arXiv:0812.3886] [SPIRES]. 
[20] U.H. Danielsson, P. Koerber and T. Van Riet, Universal de Sitter solutions at tree-level, JHEP 05 (2010) 090 [arXiv: 1003.3590] [SPIRES].

[21] P. Koerber, Stable D-branes, calibrations and generalized Calabi-Yau geometry, JHEP 08 (2005) 099 [hep-th/0506154] [SPIRES].

[22] L. Martucci and P. Smyth, Supersymmetric D-branes and calibrations on general $\mathcal{N}=1$ backgrounds, JHEP 11 (2005) 048 [hep-th/0507099] [SPIRES].

[23] P. Koerber and L. Martucci, Deformations of calibrated D-branes in flux generalized complex manifolds, JHEP 12 (2006) 062 [hep-th/0610044] [SPIRES].

[24] B. de Carlos, A. Guarino and J.M. Moreno, Complete classification of Minkowski vacua in generalised flux models, JHEP 02 (2010) 076 [arXiv:0911.2876] [SPIRES].

[25] B. de Carlos, A. Guarino and J.M. Moreno, Flux moduli stabilisation, Supergravity algebras and no-go theorems, JHEP 01 (2010) 012 [arXiv:0907.5580] [SPIRES].

[26] E. Palti, G. Tasinato and J. Ward, WEAKLY-coupled IIA Flux Compactifications, JHEP 06 (2008) 084 [arXiv:0804.1248] [SPIRES].

[27] M.R. Douglas and R. Kallosh, Compactification on negatively curved manifolds, JHEP 06 (2010) 004 [arXiv: 1001.4008] [SPIRES].

[28] D. Andriot, R. Minasian and M. Petrini, Flux backgrounds from Twists, JHEP 12 (2009) 028 [arXiv:0903.0633] [SPIRES].

[29] G.R. Cavalcanti, M. Gualtieri, Generalized complex structures on nilmanifolds, math/0404451.

[30] A.I. Malcev, On a class of homogeneous spaces, Amer. Math. Soc. Transl. 39 (1951) 1.

[31] K. Nomizu, On the cohomology of compact homogeneous spaces of nilpotent Lie groups, Ann. Math. 59 (1954) 531.

[32] J. Oprea and A. Tralle, Lecture Notes in Mathematics. Vol. 1661: Symplectic Manifolds with no Kähler Structure, Springer, Heidelberg Germany (1997).

[33] Ch. Bock, On Low-Dimensional Solvmanifolds, arXiv:0903.2926.

[34] S. Rollenske, Geometry of nilmanifolds with left-invariant complex structure and deformations in the large, arXiv:0901.3120.

[35] L. Auslander, An exposition of the structure of solvmanifolds. Part 1: Algebraic theory, Bull. Amer. Math. Soc. 79 (1973) 227.

[36] R. Campoamor-Stursberg, Some remarks concerning the invariants of rank one solvable real Lie algebras, Algebra Colloq. 12 (2005) 497.

[37] G. D. Mostow, Factor spaces of solvable groups, Ann. Math. 60 (1954) 1.

[38] G.M. Mubarakzyanov, On solvable Lie algebras, Izv. Vyssh. Uchebn. Zaved. Mat. 32 (1963) 114.

[39] P. Turkowski, Solvable Lie algebras of dimension six, J. Math. Phys. 31 (1990) 1344.

[40] P.G. Camara, A. Font and L.E. Ibáñez, Fluxes, moduli fixing and MSSM-like vacua in a simple IIA orientifold, JHEP 09 (2005) 013 [hep-th/0506066] [SPIRES].

[41] M. Graña, R. Minasian, M. Petrini and A. Tomasiello, A scan for new $\mathcal{N}=1$ vacua on twisted tori, JHEP 05 (2007) 031 [hep-th/0609124] [SPIRES]. 
[42] D. Andriot, New supersymmetric flux vacua with intermediate $\mathrm{SU}(2)$ structure, JHEP 08 (2008) 096 [arXiv:0804.1769] [SPIRES].

[43] M. Graña, R. Minasian, M. Petrini and D. Waldram, T-duality, Generalized Geometry and Non-Geometric Backgrounds, JHEP 04 (2009) 075 [arXiv:0807.4527] [SPIRES].

[44] E. Bergshoeff, R. Kallosh, T. Ortín, D. Roest and A. Van Proeyen, New Formulations of $D=10$ Supersymmetry and D8-O8 Domain Walls, Class. Quant. Grav. 18 (2001) 3359 [hep-th/0103233] [SPIRES].

[45] D. Cassani, Reducing democratic type-II supergravity on $\mathrm{SU}(3) \times \mathrm{SU}(3)$ structures, JHEP 06 (2008) 027 [arXiv: 0804.0595] [SPIRES].

[46] S. Fidanza, R. Minasian and A. Tomasiello, Mirror symmetric SU(3)-structure manifolds with NS fluxes, Commun. Math. Phys. 254 (2005) 401 [hep-th/0311122] [SPIRES].

[47] S. Kachru and A.-K. Kashani-Poor, Moduli potentials in type IIA compactifications with RR and NS flux, JHEP 03 (2005) 066 [hep-th/0411279] [SPIRES].

[48] O. DeWolfe, A. Giryavets, S. Kachru and W. Taylor, Type IIA moduli stabilization, JHEP 07 (2005) 066 [hep-th/0505160] [SPIRES].

[49] S.B. Giddings, S. Kachru and J. Polchinski, Hierarchies from fluxes in string compactifications, Phys. Rev. D 66 (2002) 106006 [hep-th/0105097] [SPIRES].

[50] K. Skenderis and M. Taylor, Branes in AdS and pp-wave spacetimes, JHEP 06 (2002) 025 [hep-th/0204054] [SPIRES].

[51] S. Console and A. Fino, On the de Rham Cohomology of solvmanifolds, arXiv:0912.2006.

[52] S.M. Salomon, Complex structures on nilpotent Lie algebras, J. Pure Appl. Algebra 157 (2001) 311.

[53] G.M. Mubarakzyanov, Classification of solvable Lie algebras of sixth order with a non-nilpotent basis element, Izv. Vyssh. Uchebn. Zaved. Mat. 35 (1963) 104.

[54] G.M. Mubarakzyanov, Certain theorems on solvable Lie algebras, Izv. Vyssh. Uchebn. Zaved. Mat. 55 (1966) 95.

[55] M. Saitô, Sous-groupes discrets des groupes résolubles, Am. J. Math. 83 (1961) 369.

[56] K. Hasegawa, Four dimensional compact solvmanifolds with and without complex analytic structures, math/0401413. 